\title{
Dynamic Global Currency Hedging
}

\author{
Bent Jesper Christensen and Rasmus T. Varneskov
}

CREATES Research Paper 2016-3 


\title{
Dynamic Global Currency Hedging*
}

\author{
Bent Jesper Christensen ${ }^{\dagger}$ \\ Aarhus University and CREATES
}

\author{
Rasmus Tangsgaard Varneskov ${ }^{\ddagger}$ \\ Northwestern University, CREATES and \\ Nordea Asset Management
}

January 18, 2016

\begin{abstract}
This paper proposes a model for discrete-time hedging based on continuous-time movements in portfolio and foreign currency exchange rate returns. In particular, the vector of optimal currency exposures is shown to be given by the negative realized regression coefficients from a one-period conditional expectation of the intra-period quadratic covariation matrix for portfolio and foreign exchange rate returns. These are labelled the realized currency betas. The model, hence, facilitates dynamic hedging strategies that depend exclusively on the dynamic evolution of the ex-post quadratic covariation matrix. These hedging strategies are suggested implemented using modern, yet simple, non-parametric techniques to accurately measure and dynamically model historical quadratic covariation matrices. The empirical results from an extensive hedging exercise for equity investments illustrate that the realized currency betas exhibit important time variation, leading to substantial economic, as well as statistically significant, volatility reductions from the proposed hedging strategies, compared to existing benchmarks, without sacrificing returns. As a result, a risk-averse investor is shown to be willing to pay several hundred basis points to switch from existing hedging methods to the proposed realized currency beta approach. Interestingly, the empirical analysis strongly suggests that the superior performance of the latter during the most recent global financial crisis of 2008 is, at least partially, funded by carry traders.
\end{abstract}

Keywords: Currency Hedging, Foreign Exchange Rates, High-frequency Data, Infill Asymptotics, Mean-Variance Analyis, Quadratic Covariation, Realized Currency Beta.

JEL classification: C14, C32, C58, G11, G15

\footnotetext{
${ }^{*}$ We are grateful to Torben G. Andersen, Ian Dew-Becker, Asbjørn Trolle Hansen, Kurt Kongsted, Ravi Jagannathan, Viktor Todorov, Claus Vorm, and seminar participants at Kellogg School of Management for many useful comments and suggestions. Financial support from Aarhus School of Business and Social Sciences, Aarhus University, the Danish Council for Independent Research | Social Sciences (FSE), and the Center for Research in Econometric Analysis of TimE Series (CREATES), funded by the Danish National Research Foundation (DNRF78), is gratefully acknowledged. Varneskov is with both Northwestern University and Nordea Asset Management. The views expressed here are those of the authors, and not necessarily any of the affiliated institutions.

${ }^{\dagger}$ Department of Economics and Business Economics, Aarhus School of Business and Social Sciences, Aarhus University, 8210 Aarhus V., Denmark. Email: bjchristensen@creates.au.dk.

${ }^{\ddagger}$ Corresponding author: Department of Finance, Kellogg School of Management, Northwestern University, Evanston, IL 60208. Email: rasmus.varneskov@kellogg.northwestern.edu.
} 
"Currency hedging is the hottest thing in investing right now."

Article headline, Business Insider UK, March, 2015.

\section{Introduction}

The potential benefits from international diversification have been recognized in the academic finance literature ever since the work of Grubel (1968), Levy \& Sarnat (1970), and Solnik (1974). Many empirical studies, however, find little, if any, statistically significant diversification benefits from investments across developed countries in more recent times, unless carried out using specific investment styles, such as size, value, and momentum strategies. ${ }^{1}$ A possible explanation for these somewhat discouraging results (seen from the perspective on an investor) is the continuing integration of international financial markets, which results in higher correlation between international assets and, thereby, diminishes the potential for harvesting diversification benefits, see, e.g., Longin \& Solnik (1995), Bekaert, Hodrick \& Zhang (2009), and Christoffersen, Errunza, Jacobs \& Langlois (2012).

Most of the aforementioned studies neglect an important component of international investments: The currency exposure implicit in the international equity portfolio holdings. In other words, international investments in a given foreign country are exposed to exchange rate movements, and investors need to decide if and how to hedge this additional risk. In practice, investment professionals often choose to hedge a certain fraction of their currency exposure, popular choices being the half-hedge and the full hedge. Some studies have analyzed hedging strategies that go beyond simple rule-ofthumb guides. In particular, Glen \& Jorion (1993), de Roon, Nijman \& Werker (2003), Campbell, de Medeiros \& Viceira (2010), Schmittmann (2010), Kroencke, Schindler \& Schrimpf (2014), and Opie \& Dark (2015) analyze diversification benefits from optimal hedging strategies based on the theory originally proposed by Anderson \& Danthine (1981), albeit with mixed empirical results. ${ }^{2}$ Whereas they all reject leaving international investments unhedged, the first two studies find no significant evidence that a static optimal hedging strategy provides diversification benefits beyond what can be achieved by fully hedging international equity investments. However, when implementing a pseudodynamic hedging strategy where the optimal currency exposure to a given foreign country depends on the level of its interest rates relative to those in the domestic country, thus mimicking some form of carry trade hedge, they find significant gains over full hedging. Campbell et al. (2010) find that a static optimal hedging strategy significantly reduces the risk of international equity investments, compared to the gains from full hedging, and a similar pseudo-dynamic hedging strategy provides additional, yet economically modest and often statistically insignificant, diversification benefits. Furthermore, their subsample analysis suggests that optimal currency exposures are quite sensitive to the specific sample

\footnotetext{
${ }^{1}$ This includes, for example, the mean-variance analyses in Britten-Jones (1999), Errunza, Hogan \& Hung (1999), Eun, Huang \& Lai (2008), Eun, Lai, de Roon \& Zhang (2010), Eun \& Lee (2010), Fama \& French (2012), Kan \& Zhou (2012), and many references therein. See also the review by Karolyi \& Stulz (2003).

${ }^{2}$ Optimal in this setting is to be understood in a mean-variance sense, i.e., as the solution to a quadratic optimization problem for an investor seeking to maximize her risk-return tradeoff.
} 
under consideration. Similar results are obtained by Schmittmann (2010) and Opie \& Dark (2015) from different countries' perspectives and across various horizons, corroborating the conclusions. Finally, Kroencke et al. (2014) take a deeper look into the diversification benefits from using traditional currency investment styles such as carry trade, momentum, and value strategies in said framework, thus promoting the pseudo-dynamic aspect of the optimal hedging strategy. They find significant diversification benefits, in particular when including foreign exchange rates for countries outside of the G10. However, the diversification benefits from their two-step procedure stem from the speculative asset allocation in the second step, not from the hedging itself, where their results resemble those of Campbell et al. (2010). Thus, they predominantly reflect the profitability of the three currency investment styles over the last 30-40 years. The present paper, on the other hand, reconsiders the first step, and so is mainly concerned with enhancing the diversification benefits from hedging strategies, conditionally on a given portfolio, not with the speculative component of currency investments. ${ }^{3}$

There are two important aspects of previous approaches to currency hedging, however, that demand further attention. First, all aforementioned studies of optimal currency exposure rely on the theoretical results from Anderson \& Danthine (1981), who assume that asset prices are observed at the same frequency as that at which the investor rebalances her portfolio, that is, the frequency at which hedging decisions are made. This implies, for example, that if an investor rebalances her portfolio at a monthly frequency, then movements in asset prices occur at monthly frequencies, as well. Hence, this approach neglects all information from asset price movements occurring at higher frequencies, e.g., daily or intra-daily. Secondly, the hedging strategies are often promoted in their static, or unconditional, form, suggesting that optimal currency exposures should be constant, often over a time span of 3040 years, and estimated using full sample information. When the hedging strategies are given a time-varying flavor, it is by conditioning on variables related to currency investment styles, such as past interest rate differentials. This approach is labelled "pseudo-dynamic" for two reasons: (1) All intertemporal movements in the optimal currency exposures are determined by slowly varying conditioning variables. Hence, no traditional time series modeling (ARMA, GARCH, or stochastic volatility) is actually performed. (2) The implementation of the hedging strategies is often in-sample, i.e., the functional link to the interest differentials is estimated using full sample information, then used for conditional hedging decisions. ${ }^{4}$ Hence, neither the static nor the pseudo-dynamic implementation of the optimal hedging strategies is designed for real-time investment decisions, and they provide inadequate descriptions of the dynamic properties of optimal currency exposures. ${ }^{5}$

\footnotetext{
${ }^{3}$ A related body of work considers optimal hedging of spot exchange rate risk using equivalent currency futures contracts in conditional frameworks resemblant of that developed by Anderson \& Danthine (1981), see, for example, Baillie \& Bollerslev (1989), Kroner \& Sultan (1993), and Bos, Mahieu \& van Dijk (2000). However, this problem is distinct from the present setting of strategic utilization of currency exposures to improve the performance of an existing portfolio.

${ }^{4}$ The implementation of the optimal hedging strategies using currency investment styles in Kroencke et al. (2014) does not suffer from (2), as the conditioning variables for the styles are contemporaneously available when the investor rebalances her portfolio, but (1) still applies, and similarly for the robustness check in Campbell et al. (2010, Section 6).

${ }^{5}$ Opie \& Dark (2015) perform a pseudo out-of-sample analysis where they compare rule-of-thumb hedges to a static optimal hedging strategy and a dynamic strategy based on a multivariate GARCH model, both implemented with rolling windows to estimate the currency exposures. Similar to Campbell et al. (2010), they show that the two optimal strategies produce
} 
The present paper addresses both caveats by introducing a new economic model for discrete time currency hedging that not only allows the assets of interest - the portfolio and foreign currencies to exhibit within-period movements, but actively utilizes the enhanced information set to construct accurate measures of optimal currency exposures. In particular, the latter are shown to be the negative realized regression coefficients from a one-period conditional expectation of the intra-period quadratic covariation matrix for portfolio and foreign currency exchange rate returns, which are labelled the realized currency betas. The model, hence, facilitates dynamic hedging strategies, depending exclusively on the dynamic evolution of the ex-post quadratic covariation matrix. This has the strong theoretical implication that interest rate differentials have no asymptotic impact on the optimal currency demands for a given international portfolio, in stark contrast with existing hedging theory, e.g., Anderson \& Danthine (1981), Glen \& Jorion (1993), and Campbell et al. (2010). Moreover, as the proposed strategies do not rely on information about local trends in currencies in their construction, they are clearly different from traditional currency investment styles, such as carry, momentum, and value trading. From a theoretical perspective, the development of the realized currency beta-based hedging framework involves establishing new results for optimal currency exposures based on the notion of quadratic covariation measures and infill asymptotic limits. From a practical perspective, the theory suggests that an investor should sample as frequently as possible within fixed time intervals between portfolio rebalances to construct accurate estimates the quadratic covariation matrix and, subsequently, model its dynamics. Hence, this paper proposes to implement the new hedging strategies using modern, yet simple, non-parametric techniques to accurately measure and dynamically model historical quadratic covariation matrices, imposing only few parametric restrictions on the underlying processes.

The new dynamic hedging strategies are analyzed in an extensive empirical exercise, covering multiple different international equity portfolios, rebalancing horizons, and time periods. This produces several novel and striking results that may be summarized as follows:

(i) There is substantial time variation in the optimal currency exposures. For example, the optimal exposures to the Swiss Franc and Japanese Yen are both essentially zero for decades in the first part of the full sample period, covering 1975 through August 2014, but increase dramatically during the last 16, respectively 7 years, which include the most recent financial crisis and subsequent European debt crisis. The Euro, on the other hand, switches status from being a hedge when introduced in 1999, to being a currency with zero optimal exposure, and finally becoming a speculative currency during the most recent financial crisis, a status it then retains throughout. In addition to pronounced general patterns, temporary spikes and elevations in optimal currency exposures can be tied to important economic events, such as the collapse of Lehman Brothers, interventions of the Swiss National Bank, and falling stock markets in August 2011.

(ii) While the results from Campbell et al. (2010) are corroborated - a static optimal hedging strategy

the lowest portfolio volatility, but also display statistically indistinguishable performance. That is, they find no additional improvements from actual dynamic modeling. However, despite considering the second caveat, their framework, as well as analysis, does not treat the issue of sampling versus rebalancing frequency. 
significantly reduces the risk of international equity investments compared to the gains from full hedging - the proposed dynamic hedging strategies, based on realized currency betas, provide statistically significant volatility reductions compared to both. The volatility reductions are substantial and are generated without sacrificing returns.

(iii) Using a long time span data set of daily observations, covering January 1975 through August 2014, for rebalancing horizons ranging from one week to one quarter, a risk-averse investor with a standard level of risk aversion relative to the literature is shown to be willing to pay 400-500 basis points to switch from a fully hedged static position to the proposed dynamic hedging strategies. Moreover, compared to the static optimal hedging strategies from Anderson \& Danthine (1981) and Campbell et al. (2010), the investor is wiling to pay 170-300 basis points to make the switch.

(iv) The addition of a synthetic carry trade currency to the set of hedging currencies, thus mimicking the pseudo-dynamic hedging strategies in the literature, provides no further volatility reductions. Moreover, its impact on portfolio returns is ambiguous and small in magnitude.

(v) A comparison between the returns to the dynamic realized currency beta hedging strategy and those to traditional currency investment styles shows that the former is negatively correlated with carry trade, and only modestly correlated with currency momentum and value investments. Interestingly, the analysis strongly suggests that carry traders, at least partially, fund the strong performance of the proposed dynamic strategy during the most recent financial crisis.

(vi) The realized currency betas display similar patterns for different equity portfolios. However, they also exhibit persistent differences in the levels of optimal currency exposures as well as different temporary elevations and spikes in response to important economic events, suggesting the need to customize the dynamic hedging strategy to the equity portfolio under consideration.

(vii) The hedging results are corroborated and expanded upon using a carefully collected data set of intra-daily observations covering September 2005 through August 2014 on multiple equity index and currency futures from different exchanges and with different trading hours. Again using rebalancing horizons of one week and one month, the intra-daily data are used to construct more precise measures of quadratic covariation, leading to dynamic hedging strategies worth more than 800 basis points relative to a full static hedge, and 150-200 basis points compared to a dynamic hedging strategy implemented using quadratic covariation measures based on daily data.

All of the findings (i)-(vii) are new to the literature on global currency hedging. In particular, the empirical hedging results from the long span analysis corroborate and go well beyond those in existing studies, such as Glen \& Jorion (1993) and Campbell et al. (2010), not only by showing how dynamic hedging strategies can be designed to obtain better risk-return tradeoffs than full hedging and static optimal procedures, but also by estimating the economic gains from such strategies to a riskaverse investor, documenting pronounced and important time-variation in optimal currency exposures, 
showing how this links to key economic events, and describing common patterns and differences in the optimal currency exposures across various international equity portfolios.

The finding that dynamic hedging strategies based on intra-daily rather daily data improves portfolio performance is consistent with Fleming, Kirby \& Ostdiek (2001, 2003), who study dynamic asset allocation between S\&P 500, Treasury bond, and gold futures. ${ }^{6}$ However, in addition to the present analysis being one of hedging rather than asset allocation, the elicitation of gains from intra-daily data in the international investments and currency trading case is more challenging than in their singlecountry analysis, due to assets being traded on different exchanges with only partially overlapping trading hours. Furthermore, our results demonstrating that dynamic rather than static modeling of exchange rate covariances leads to economic gains for a risk-averse investor are consistent with findings of Della Corte, Sarno \& Tsiakas (2009), who analyze asset allocation between fixed income and currencies by applying different univariate dynamic models to monthly data.

Even though individual currencies have traditionally been viewed as poor investments vehicles with low return and high volatility, there has been a recent surge of academic papers in a separate strand of the exchange rate modeling literature, showing that systematic currency trading, in particular carry trade, momentum, and value investments, may be highly profitable, even on a risk-adjusted basis, see, for example, the recent contributions by Lustig \& Verdelhan (2007), Brunnermeier, Nagel \& Pedersen (2009), Burnside, Eichenbaum, Kleshchelski \& Rebelo (2011), Lustig, Roussanov \& Verdelhan (2011, 2014), Menkhoff, Sarno, Schmeling \& Schrimpf (2012a, 2012b), Moskowitz, Ooi \& Pedersen (2012), Asness, Moskowitz \& Pedersen (2013), and many references therein. The dynamic hedging strategies proposed in the present paper similarly constitute systematic trading opportunities in currencies. However, they are designed with the specific purpose of improving the performance of an already existing portfolio. Moreover, as the realized currency betas are asymptotically invariant to changes in interest rate differentials and only use information about the covariance between foreign exchange rate and portfolio returns in their construction, that is, no information about local trends in the former, they are clearly different from traditional investment styles. In fact, as noted in (iv) above, there are no hedging gains to carry trade, once the proposed dynamic strategies are implemented. Moreover, the findings in (v) not only suggest that the proposed dynamic strategies may provide a hedge for carry trade, as well, its favorable correlation properties suggest that there may be intriguing opportunities to combine the four different methods in designing tactical foreign exchange rate trading.

The outline of the paper is as follows. Section 2 introduces the new economic model and the assumptions, then derives the theoretical foundation for the proposed dynamic currency hedging strategies. Section 3 discusses the non-parametric implementation procedure. Section 4 introduces the long-span data set of daily observations and provides empirical evidence of time variation in the optimal currency exposures. The risk-return performance and economic benefits from implementing different hedging

\footnotetext{
${ }^{6}$ It is also consistent with Andersen, Bollerslev, Diebold \& Labys (2003), who consider VaR estimation using 30-minute returns on two currencies, and with Liu (2009), Chiriac \& Voev (2011), and Varneskov \& Voev (2013), who study meanvariance analysis using intra-daily data on DJIA stocks. However, none of these studies considers the interaction between equity investments and currency exposures, nor do they use intra-daily data to design currency hedging strategies.
} 
strategies are examined in Section 5. Sections 6 relates the returns to the dynamic hedging strategies to those from traditional currency investment styles. The informational content and interpretation of realized currency betas are elaborated upon in Section 7. Section 8 extends the analysis to include intra-daily data. Section 9 generalizes the theoretical results to include jumps and briefly discusses their role, and Section 10 concludes. The appendix contains the proofs, and a supplementary web appendix, Christensen \& Varneskov (2015), provides results from various robustness checks, along with implementation details.

\section{The Dynamic Modeling Framework}

This section introduces a multi-period model for discrete-time hedging based on continuous-time within-period movements in the underlying portfolio and foreign exchange rate returns. The model is intended to capture the decision problem of an investor who rebalances, or re-hedges, her portfolio in fixed time intervals, but observes both portfolio and exchange rate movements within each interval. Optimal currency exposures are established using infill asymptotic theory for a general class of continuous-time price processes. The discrete time framework follows along the lines of Anderson \& Danthine (1981) and Campbell et al. (2010). However, as shown below, allowing for the continuous-time within-period movements in the processes of interest not only generalizes the framework considerably, it also simplifies the optimal hedging decision.

\subsection{Discrete Time Decision Making}

Suppose that for each discrete point in time $t=1,2, \ldots, T$, an investor holds a position $w_{c, t}$ in country $c$ 's equities, $c=0, \ldots, C$, from time $t$ until $t+1$, when the holding pays a gross continuously compounded return of $R_{c, t+1} \cdot{ }^{7}$ For simplicity, let $c=0$ index the home country, which is assumed to be the US, and let $S_{c, t+1}$ be the corresponding time $t+1$ spot exchange rate in USD per foreign currency unit.

In this setting, the US investor earns an unhedged return of $R_{c, t+1}^{u}=R_{c, t+1} S_{c, t+1} / S_{c, t}$ on her country $c$ investment. To hedge the latter against currency risk, the investor buys a holding of the one-period forward exchange rate $F_{c, t}$, also measured in USD per foreign currency unit, at time $t$ in country $c$. Let $\theta_{c, t}$ be the dollar value of this holding per USD invested in the equity portfolio. Thus, the investor gets to exchange $\theta_{c, t} / S_{c, t}$ units of $R_{c, t+1} w_{c, t} / S_{c, t}$ back into USD at the exchange rate $F_{c, t}$, and the remaining $\left(R_{c, t+1} w_{c, t} / S_{c, t}-\theta_{c, t} / S_{c, t}\right)$ units of foreign currency at the spot exchange rate $S_{c, t+1}$. This suggests writing the hedged portfolio return as

$$
R_{t+1}^{h}=\sum_{c=0}^{C} w_{c, t} R_{c, t+1}^{u}+\sum_{c=0}^{C} \theta_{c, t} \frac{F_{c, t}}{S_{c, t}}-\sum_{c=0}^{C} \theta_{c, t} \frac{S_{c, t+1}}{S_{c, t}} .
$$

\footnotetext{
${ }^{7}$ The exposition is throughout laid out for equities. This may without loss of generality, however, be changed to other assets held in foreign countries, such as corporate bonds, commodities, derivates, etc., as long as the assumptions on the included assets, as outlined below, are satisfied.
} 
Notice that the choice of domestic hedge ratio, $\theta_{0, t}$, is arbitrary since $S_{0, t}=F_{0, t}=1$ for all $t$. Hence, for ease of exposition, the hedge ratios are normalized to sum up to one, implying that

$$
\sum_{c=0}^{C} w_{c, t}=1, \quad \theta_{0, t}=1-\sum_{c=1}^{C} \theta_{c, t}
$$

for all $t$. Maintaining an assumption of absence of arbitrage throughout, it follows by the covered interest rate parity $(\mathrm{CIP})$ that $F_{c, t} / S_{c, t}=\left(1+I_{0, t}\right) /\left(1+I_{c, t}\right)$, where $I_{c, t}, c=0, \ldots C$, denotes the nominal short-term risk-free interest rate. This identity may be inserted directly in (1) above.

The form of the portfolio return in (1) also allows for speculative positions in exchange rates if, for example, the currency demand $\theta_{c, t}$ is driven by a model for local trends in $S_{c, t}$, regardless of its correlation with the portfolio return. Hence, to avoid confusion, the label "hedging" in this paper signifies that currency demands are determined with the explicit objective of reducing the risk of the portfolio return, thus seeking currencies with favorable correlation properties. In other words, currency hedgers and speculators are distinguished according to whether they emphasize correlation properties or local trends, respectively, when selecting foreign exchange rate exposure.

\subsection{Intra-period Dynamics}

Suppose that the processes of interest - equities, currencies, and bonds - are defined on a filtered probability space, $\left(\Omega, \mathcal{F},\left(\mathcal{F}_{\tau}\right), \mathbb{P}\right)$, where $\tau \in[t, t+1]$ is the within-period time indicator. In the absence of arbitrage, prices are assumed to follow semimartingales, see, e.g., Back (1991) and Delbaen $\&$ Schachermayer (1994). Hence, let $P_{c, \tau}$ denote the price of the equity holdings in country $c$, measured in local currency, and $B_{c, t}$ the price of a country $c$-denominated risk-less bond. Then, for $c=0, \ldots, C$, the system of equity, currency, and bond prices is assumed to obey

$$
\begin{aligned}
d P_{c, \tau} / P_{c, \tau} & =\mu_{c, \tau} d \tau+\sigma_{c, \tau} d W_{c, \tau} \\
d S_{c, \tau} / S_{c, \tau} & =\alpha_{c, \tau} d \tau+\varphi_{c, \tau} d Y_{c, \tau} \\
d B_{c, \tau} / B_{c, \tau} & =\lambda_{c, \tau} d \tau
\end{aligned}
$$

where $\left(\mu_{c, \tau}, \alpha_{c, \tau}\right)$ and $\left(\sigma_{c, \tau}, \varphi_{c, \tau}\right)$ capture the within-period drift and stochastic volatility of equity and currency returns; $W_{c, \tau}$ and $Y_{c, \tau}$ are standard Brownian motions; and $\lambda_{c, \tau}$ models the instantaneous return from holding a short-term risk-less bond. ${ }^{8}$ The formal theoretical analysis necessitates the following additional structure on the within-period price system:

Assumption 1. Suppose the components of the intra-period price system (3)-(5) satisfy the following conditions for all $(c, k) \in\{0, \ldots, C\}^{2}$ :

(a) $\mu_{c, t}$ and $\alpha_{c, \tau}$ are $\mathcal{F}_{\tau}$-predictable and locally bounded;

\footnotetext{
${ }^{8}$ The time $t$ subscript is dropped for notational simplicity when describing the intra-period price system (3)-(5), since the representation is valid for all intervals, with $\tau \in[t, t+1], t=1, \ldots, T$.
} 
(b) $\lambda_{c, \tau}$ is $\mathcal{F}_{t}$-predictable and locally bounded;

(c) $\sigma_{c, \tau}$ and $\varphi_{c, \tau}$ are $\mathcal{F}_{\tau}$-adapted, locally bounded, càdlàg, and strictly greater than zero;

(d) the quadratic covariation between $d W_{c, \tau}$ and $d W_{k, \tau}, c \neq k$, is defined as $d\left[W_{c}, W_{k}\right]_{\tau}=\sigma_{c, k, \tau} d \tau$ where $\sigma_{c, k, \tau}$ is $\mathcal{F}_{\tau}$-adapted, locally bounded, and càdlàg;

(e) the quadratic covariation between $d Y_{c, \tau}$ and $d Y_{k, \tau}, c \neq k$, is defined as $d\left[Y_{c}, Y_{k}\right]_{\tau}=\varphi_{c, k, \tau} d \tau$ where $\varphi_{c, k, \tau}$ is $\mathcal{F}_{\tau}$-adapted, locally bounded, and càdlàg;

(f) the quadratic covariation between $d W_{c, \tau}$ and $d Y_{k, \tau}$ is defined as $d\left[W_{c}, Y_{k}\right]_{\tau}=\psi_{c, k, \tau} d \tau$ where $\psi_{c, k, \tau}$ is $\mathcal{F}_{\tau}$-adapted, locally bounded, and càdlàg.

The present setting generalizes the previously developed framework for deriving optimal currency exposure in Anderson \& Danthine (1981), Glen \& Jorion (1993), de Roon et al. (2003), and Campbell et al. (2010), by allowing for stochastic drift and volatility, as well as intra-period movements in equites and currencies. The latter are assumed to belong to a general class of continuous Brownian semimartingales, which is commonly used in the literature on high-frequency volatility estimation since it nests many continuous-time models in financial economics. ${ }^{9}$ For example, the class accommodates the widely documented presence of leverage effects, i.e., non-zero correlation between innovations in the price process and the stochastic volatility process. ${ }^{10}$ The whole modeling system implies that in a given time interval, $[t, t+1]$, between the rebalancing times of the portfolio of equities, currencies, and bonds, asset prices are allowed to evolve according to continuous trajectories, which, as will be laid out clearly below, is important for the investor's optimal currency position.

Before deriving the optimal currency exposures, however, it is important to characterize the path of the hedged portfolio return at each time $\tau \in[t, t+1]$. Hence, with $V_{\tau}$ denoting the value of the hedged portfolio at time $\tau$, the evolution of its instantaneous return may be described using (3)-(5) as

$$
\frac{d V_{\tau}}{V_{\tau}}=\sum_{c=0}^{C} w_{c, t} \frac{d\left(P_{c, \tau} S_{c, \tau}\right)}{P_{c, \tau} S_{c, \tau}}+\sum_{c=0}^{C} \theta_{c, t} \frac{d\left(B_{0, \tau} / B_{c, \tau}\right)}{\left(B_{0, \tau} / B_{c, \tau}\right)}-\sum_{c=0}^{C} \theta_{c, t} \frac{d S_{c, \tau}}{S_{c, \tau}} .
$$

As in Campbell et al. (2010), it simplifies the problem to work in logarithms and use matrix notation. Hence, let $r_{t+1}^{h}=\ln \left(R_{t+1}^{h}\right)$ and $x_{c, \tau}=\ln \left(X_{c, \tau}\right)$ for $X=\{P, S, V\}$. Similarly, let $\boldsymbol{w}_{t}=\left(w_{0, t}, \ldots, w_{C, t}\right)^{\prime}$ be the $(C+1) \times 1$ vector of portfolio weights and $\boldsymbol{\Theta}_{t}=\left(\theta_{0, t}, \ldots, \theta_{C, t}\right)^{\prime}$ the corresponding $(C+1)$ dimensional vector of currency hedging positions; $\boldsymbol{x}_{\tau}=\left(x_{0, \tau}, \ldots, x_{C, \tau}\right)^{\prime}$ for $x=(p, s, \lambda)^{\prime}$; and, finally, $\boldsymbol{\lambda}_{0, \tau}=\boldsymbol{\iota} \lambda_{0, \tau}$ for a $(C+1) \times 1$ vector of ones, $\iota{ }^{11}$ Finally, to explicitly capture the fact that an investor can alter her currency exposure by lending and borrowing (going long or short in bonds

\footnotetext{
${ }^{9}$ For early references, consult Comte \& Renault (1998), Andersen, Bollerslev, Diebold \& Ebens (2001), Andersen, Bollerslev, Diebold \& Labys (2001, 2003), Meddahi (2002), Hansen \& Lunde (2006), and Bandi \& Russell (2008). Furthermore, see, e.g., Andersen, Bollerslev \& Diebold (2010) and Andersen \& Benzoni (2012) for reviews and additional references.

${ }^{10}$ See, e.g., Black (1976), Christie (1982), Engle \& Ng (1993), and Yu (2005).

${ }^{11}$ In general, logarithmic transformations are written with lowercase letters, and matrices expressed in boldface.
} 
or forward contracts), define $\boldsymbol{\beta}_{t} \equiv\left(\beta_{0, t}, \ldots, \beta_{C, t}\right)^{\prime}=\boldsymbol{w}_{t}-\boldsymbol{\Theta}_{t}$ as the selected currency exposure in excess of the implicit exposure in the international portfolio holdings. For example, $\beta_{c, t}=0$ and $\beta_{c, t}=w_{c, t}$ correspond to having fully respectively unhedged investments in country $c$ 's equities. In general, $\beta_{c, t}>0$ implies that the investor demands exposure to currency $c$ and, equivalently, she wants to be underexposed if $\beta_{c, t}<0$. Note that (2) implies $\boldsymbol{\beta}_{t}^{\prime} \boldsymbol{\iota}=0$, that is, the dynamic currency hedging portfolio is a zero investment, meaning that all long positions in currencies are financed by shorting bonds in funding currencies, similarly as in traditional currency investment styles. Finally, a weak regularity condition is imposed on the elements of $\boldsymbol{w}_{t}$ and $\boldsymbol{\Theta}_{t}$.

Assumption 2. For all $t=1, \ldots, T$, $\sup _{c=0, \ldots, C}\left|w_{c, t}\right|+\sup _{c=0, \ldots, C}\left|\theta_{c, t}\right|<\infty$.

Assumption 2 innocuously states that both the equity portfolio weight in and currency exposure to country $c, c=0, \ldots, C$, must be finite. ${ }^{12}$ Finally, the following lemma provides a representation result for the within-period log-returns to the hedged portfolio, $d v_{\tau}$.

Proposition 1. Suppose the representation (6) and Assumptions 1-2 hold, then

$$
d v_{\tau}=\boldsymbol{w}_{t}^{\prime}\left(d \boldsymbol{p}_{\tau}+\boldsymbol{\lambda}_{0, \tau} d \tau-\boldsymbol{\lambda}_{\tau} d \tau\right)+\boldsymbol{\beta}_{t}^{\prime}\left(d \boldsymbol{s}_{\tau}-\boldsymbol{\lambda}_{0, \tau} d \tau+\boldsymbol{\lambda}_{\tau} d \tau\right)+\Sigma_{\tau}^{h} d \tau+o_{p}(d \tau),
$$

where $\Sigma_{\tau}^{h}$ is $\mathcal{F}_{\tau}$-adapted, locally bounded, and càdlàg.

Proof. See Appendix A.1.

Proposition 1, similarly to the representation in Campbell et al. (2010, Equation (1)), illustrates the decomposition of the hedged log-return into three components; the first term is the instantaneous excess return on a fully hedged portfolio; the second term represents the instantaneous excess return on currencies, which depends on the selected exposure, $\boldsymbol{\beta}_{t}$; and the last term is a Jensen's inequality correction. However, unlike in the corresponding framework in Anderson \& Danthine (1981) and Campbell et al. (2010), the instantaneous log-return on the hedged portfolio is allowed to evolve stochastically in the interval $\tau \in[t, t+1]$, implying that the one-period log-return may be written as

$$
r_{t+1}^{h}=\int_{t}^{t+1} d v_{\tau}, \quad t=1, \ldots, T
$$

thus illustrating the link between the two frameworks. Equation (7) suggests that the one-period log-return on a hedged portfolio may be interpreted as the sum of returns at a higher frequency. For an investor with a monthly investment horizon, this could, e.g., be a sum of daily log-returns.

Remark 1. While the zero net exposure condition $\boldsymbol{\beta}_{t}^{\prime} \iota=0$ demonstrates that the implicit domestic USD exposure, or hedging position, is determined once the exposures to the $C$ foreign exchange rates

\footnotetext{
${ }^{12}$ Strictly speaking, the condition $\sup _{c=0, \ldots, C}\left|\theta_{c, t}\right|<\infty$ should be shown endogenously in the model, since $\theta_{c, t}$ will depend on the components of the intra-period price system (3)-(5). However, by assuming it from the outset, rather than showing it endogenously, the proofs of Propositions 1 through 4 below may be shortened considerably.
} 
are set, the former has no impact on $d v_{\tau}$, since $d s_{0, \tau}=0, \forall \tau \in[t, t+1]$, and the interest differential is also trivially zero in this case.

Remark 2. It is possible to extend the vector price system (3)-(5) to accommodate jumps. This leads only to minor changes in the interpretation of the results, as discussed in Section 9 below.

\subsection{Optimal Dynamic Currency Exposure}

The optimal dynamic selection of currency exposure requires the choice of an appropriate objective function. Usually, in portfolio selection problems, this involves choosing the portfolio weights such that they minimize portfolio variance subject to certain constraints. Similarly to the one-period log-return (7), which is measured by cumulating returns at higher frequency, a measure of its variance must also reflect the stochastic intra-period movements in $d v_{\tau}$. A natural measure of variability in this setting is quadratic variation, see, e.g., Andersen et al. (2010). Formally, suppose the intra-period hedged $\log$-return $d v_{\tau}$ is observed on a discrete partitioning $\tau_{i}$ of the interval, $t=\tau_{0}<\tau_{1}<\cdots<\tau_{n}=t+1$. Then the quadratic variation of $r_{t+1}^{h}$ is defined as

$$
\left[d v_{\tau}\right]_{t+1}=\operatorname{plim}_{n \rightarrow \infty} \sum_{i=1}^{n} d v_{\tau_{i}}^{2}=\int_{t}^{t+1} d v_{\tau}^{2}
$$

for $\sup _{i}\left\{\tau_{i+1}-\tau_{i}\right\}=0$ as $n \rightarrow \infty$, see, e.g., Jacod \& Shiryaev (2003) for details. ${ }^{13}$ Conceptually, quadratic variation is an ideal return variability measure as it captures the entire realized ex-post variability of the hedged log-returns. Furthermore, its use simplifies computation of the optimal currency exposure considerably, as illustrated by the following proposition:

Proposition 2. Suppose the conditions of Proposition 1 holds, then as $n \rightarrow \infty$,

$$
\left[d v_{\tau}\right]_{t+1}=\left[\boldsymbol{w}_{t}^{\prime} d \boldsymbol{p}_{\tau}+\boldsymbol{\beta}_{t}^{\prime} d \boldsymbol{s}_{\tau}\right]_{t+1}+o_{p}(1)
$$

Proof. See Appendix A.2.

Proposition 2 demonstrates that the quadratic variation of the one-period hedged log-return depends only on the quadratic variations of the fully hedged log-return and the total currency exposure return, and on their quadratic covariation. Hence, there is no impact from movements in nominal shortterm risk-free interest rate differentials, or the Jensen's inequality induced term $\Sigma_{\tau}^{h} d \tau$. This distinct advantage of the proposed within-period model for equities, currencies, and bonds is due to the fact that drift components have no asymptotic impact on quadratic variation in the infill asymptotic limit. As a result, Proposition 2 provides a variance measure that contrasts starkly with the corresponding longspan variance measure used for the development of the existing currency hedging theory by Anderson

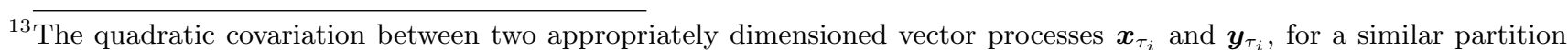
of the sample $\tau_{i} \in[t, t+1], i=0, \ldots, n$, is analogously defined as $[\boldsymbol{x}, \boldsymbol{y}]_{t+1}$, that is, as the probability limit of a sum of their outer-products as the distance between adjacent observations tend to zero. 
\& Danthine (1981), and subsequently applied in Glen \& Jorion (1993), de Roon et al. (2003), and Campbell et al. (2010), and which depends on period-by-period movements in short-term interest rate differentials.

Since the vector of dynamic net currency exposures, $\boldsymbol{\beta}_{t}$, represents a zero-investment portfolio, it suffices to determine the $C \times 1$ vector of foreign currency exposures $\tilde{\boldsymbol{\beta}}_{t}=\left(\beta_{1, t}, \ldots, \beta_{C, t}\right)^{\prime}$, as this spans the unique elements of $\boldsymbol{\beta}_{t}$. Formally, and consistently with our distinction between foreign exchange hedgers and speculators, exposures are selected to minimize the one-period conditional quadratic variation of the hedged log-return, that is, as

$$
\tilde{\boldsymbol{\beta}}_{t}^{*}=\underset{\boldsymbol{\beta}_{t} \mid \boldsymbol{w}_{t}}{\operatorname{argmin}} \mathcal{L}_{t}\left(\boldsymbol{\beta}_{t}, \boldsymbol{w}_{t}\right), \quad \mathcal{L}_{t}\left(\boldsymbol{\beta}_{t}, \boldsymbol{w}_{t}\right)=\frac{1}{2} \mathbb{E}_{t}\left[\left[d v_{\tau}\right]_{t+1}\right] .
$$

Before stating the optimality result, let $\tilde{\boldsymbol{s}}_{\tau}=\left(s_{1, \tau}, \ldots, s_{C, \tau}\right)^{\prime}$ denote the vector of currencies corresponding to the unique exposures $\tilde{\boldsymbol{\beta}}_{t}$. Then,

Proposition 3. Suppose the conditions of Proposition 2 hold and that $\mathbb{E}_{t}\left[\left[d \tilde{\boldsymbol{s}}_{\tau}\right]_{t+1}\right]$ is positive definite for all $t=1, \ldots, T$, then the limiting unique optimal currency exposures are determined by

$$
\tilde{\boldsymbol{\beta}}_{t}^{*}=-\mathbb{E}_{t}\left[\left[d \tilde{\boldsymbol{s}}_{\tau}\right]_{t+1}\right]^{-1} \mathbb{E}_{t}\left[\left[\boldsymbol{w}_{t}^{\prime} d \boldsymbol{p}_{\tau}, d \tilde{\boldsymbol{s}}_{\tau}\right]_{t+1}\right]
$$

Proof. See Appendix A.3.

Proposition 3 demonstrates that the vector of optimal currency exposures is the negative vector of realized regression coefficients from an implicit projection of the fully hedged log-return on the vector of foreign exchange rate innovations, which is embedded in the one-period conditional expectation of the quadratic covariation matrix. This former is labelled the realized currency beta, in analogy with the market exposure measured by the CAPM beta. However, it is stressed that while the market beta reflects the uncertainty of a given asset in terms of its sensitivity to market movements, the realized currency beta reflects the hedging potential from having currency exposure in a given equity portfolio and is, as a result, not a deep characteristic of a currency. This property is discussed in greater detail in Section 7 below, where a generic benchmark realized currency beta is proposed

Proposition 3 suggests that realized currency betas may be computed dynamically using only withinperiod equity- and foreign exchange rate data by first obtaining a times series of their quadratic covariation estimates, then specifying an appropriate dynamic model for these, to obtain one-stepahead conditional expectations. As such, this is a highly desirable property, since it implies that the optimal currency exposure is not only asymptotically invariant to short-term interest rate differentials, but also to the validity of the CIP, which is used to substitute out forward rates with interest rate differentials in (1) and (6). Akram, Rime \& Sarno (2008) find that the CIP holds approximately at daily or lower frequencies, which suggests that both forward and interest rate returns may be used to evaluate the return performance of the optimal hedging strategies ex-post, as long as the investor rehedges her currency exposures at sufficiently sparse intervals. However, the invariance of the optimal 
currency exposures themselves to short-term interest rates differentials and the CIP clearly separates them from the popular carry trade investments, which are designed with long positions in baskets of currencies with high short-term interest rates and short in baskets of currencies with low interest rates, resting on the failure of the uncovered interest rate parity.

Optimal currency exposures may, of course, be determined using a generalized, or different, objective function. For example, one may choose $\mathcal{L}_{t}\left(\tilde{\boldsymbol{\beta}}_{t}, \boldsymbol{w}_{t}\right)=(1-\zeta) / 2 \mathbb{E}_{t}\left[\left[d v_{\tau}\right]_{t+1}\right]+\zeta\left(\mu_{r}-\mathbb{E}_{t}\left[r_{t+1}^{h}\right]\right)$, for some target return $\mu_{r}$, if the investor seeks to preserve a speculative element in her foreign exchange rate positions. Notice, however, that the solution to the resulting minimization problem will, in general, lead to dynamic hedging strategies that depend on the first moment of the fully hedged log-returns, which cannot be estimated consistently using only intra-period observations, see Jacod (2012), and thus requires long-span modeling of the conditional means of the series, which may present a harder challenge than the modelling of conditional covariances. Instead, choosing currency exposures based on (9) not only simplifies implementation, but also allows for a direct assessment of the economic value attainable from precisely measuring and modelling the covariances between foreign exchange rates and portfolio returns. The resulting realized currency beta hedging strategies may, thus, be viewed as an alternative to traditional currency investment styles, such as carry, momentum, and value trading strategies, which rely solely on the modeling of local trends, rather than covariances. Empirical comparisons are made in Section 6 below.

Remark 3. One may consider imposing constraints on the optimal currency exposures, similar to, e.g., Jagannathan $\&$ Ma (2003). This may have stabilizing effects on non-linear transformations of the quadratic covariation estimates, in particular if the number of currencies, $C$, is large, through reduction of the impact of measurement errors. However, since the theory developed in Propositions 1-3 suggests utilizing information from observations sampled at a higher frequency than that at which hedging decisions are made, the dynamic modeling procedure, introduced below, is designed to smooth out remaining measurement errors, and since $C=6$ in the empirical application, the introduction of constraints in the present setting is unlikely to improve portfolio performance further. Still, a deeper investigation of hedging decisions under constraints is an interesting direction for further research.

Remark 4. Although the exposition is given from the perspective of a US investor, it is important to note that the realized currency betas are dynamically invariant to base currency. This implies that, e.g., a UK investor with the same equity portfolio will be choosing identical optimal currency exposures. This invariance result is formally shown in the supplementary appendix.

\section{Estimation Theory for Optimal Currency Exposures}

Dynamic implementation of the proposed realized currency beta hedging strategy requires both estimation of the latent quadratic covariance matrix over each discrete time interval between portfolio rebalances, and subsequent dynamic modeling of the covariance matrices. Hence, two different nonparametric approaches to quadratic covariation estimation, which may be applied to data sampled at 
different frequencies, are discussed first. Second, a simple filtering procedure for the construction of one-period-ahead conditional expectations of the quadratic covariation matrix is then detailed.

\subsection{Measuring Quadratic Covariation}

Suppose that the vector $\boldsymbol{x}_{\tau_{i}}=\left(\boldsymbol{w}_{t}^{\prime} d \boldsymbol{p}_{\tau_{i}}, d \tilde{\boldsymbol{s}}_{\tau_{i}}^{\prime}\right)^{\prime}$ is observed at the $n+1$ discrete time points from the portfolio rebalancing at $t$ to the next, that is, at $\tau_{i} \in[t, t+1], i=0, \ldots, n$, then the realized covariance estimator, introduced by Andersen et al. (2003) and Barndorff-Nielsen \& Shephard (2004a), represents the empirical approximation to the computations (9). Formally, the estimator may be defined as

$$
R C(\boldsymbol{x})=\sum_{i=1}^{n} \Delta \boldsymbol{x}_{\tau_{i}} \Delta \boldsymbol{x}_{\tau_{i}}^{\prime},
$$

where $\Delta=1-L$ is the usual first difference operator. Under mild conditions on the vector price system in (3)-(5), cf. Assumption $1, R C(\boldsymbol{x}) \stackrel{\mathbb{P}}{\rightarrow}[\Delta \boldsymbol{x}]_{t+1}$ for $\sup _{i}\left\{\tau_{i+1}-\tau_{i}\right\}=0$ as $n \rightarrow \infty$. Furthermore, its associated central limit theory demonstrates that convergence occurs at the optimal rate, $n^{1 / 2}$, to a mixed Gaussian distribution, and that its asymptotic variance achieves the lower bound for the estimation problem, see, e.g., the discussion in Mykland (2010). Implicit in these statements, however, is that the individual entries in $\boldsymbol{x}_{\tau_{i}}$ are observed synchronously and without measurement errors. This approximation may not be too damaging if the rebalancing horizon is, for example, weekly or monthly, and the intra-period observations are recorded daily or even intra-daily at sufficiently sparse intervals. ${ }^{14}$ If the data are sampled intra-daily at higher frequencies, on the other hand, market microstructure (MMS) effects and non-synchronicity related errors drive a wedge between the observed equity prices and exchange rates and their theoretical counterparts, leading the individual entries of standard covariance matrix estimators such as realized covariance to explode. ${ }^{15}$ Hence, if the data are available intra-daily at frequencies higher than the conventional five minute rule-of-thumb, e.g., Andersen \& Bollerslev (1998), it is pertinent to use an estimator that actively mitigates the impact from these measurement errors while maintaining good efficiency properties. A class of estimators fitting these requirements is the flat-top realized kernels, proposed by Varneskov $(2015 a, 2015 b)$.

The notion of measurement errors may be quantified as follows: Let the observable, synchronized, intra-daily observations follow an additive noise model of the form $\boldsymbol{y}_{\tau_{i}}=\boldsymbol{x}_{\tau_{i}}+\boldsymbol{u}_{\tau_{i}}$, where $\boldsymbol{u}_{\tau_{i}}$ summarizes the effects from an array of market imperfections, including synchronization errors, and referred to as MMS noise. ${ }^{16}$ Next, let $\Gamma_{h}(\boldsymbol{y})=\sum_{i=|h|+1}^{n} \Delta \boldsymbol{y}_{\tau_{i}} \Delta \boldsymbol{y}_{\tau_{i-|h|}}$ for $h \geq 0$ and $\Gamma_{h}(\boldsymbol{y})=\Gamma_{-h}(\boldsymbol{y})^{\prime}$ for $h<0$ be the realized autocovariance of $\boldsymbol{y}$ for a given lag $h$. The class flat-top realized kernels is designed

\footnotetext{
${ }^{14}$ It is not recommended to use much more sparse sampling than daily since the asymptotic approximation of negligible drift, or local trends, may be poor at such frequencies. If the series display non-negligible drift, this obviously needs to be taken into account when computing the quadratic covariation estimates.

${ }^{15}$ See, e.g., the discussions in Hansen \& Lunde (2006), Voev \& Lunde (2007), and Bandi \& Russell (2008).

${ }^{16}$ Besides synchronization errors caused, e.g., by irregular and non-synchronous observation times, the MMS noise captures both exogenous effects, such as bid-ask bounce movements, and endogenous effects, such as asymmetric information and strategic learning among market participants. See Varneskov (2015b) for a detailed discussion.
} 
to eliminate the noise-induced bias and variance of the realized covariance estimator by weighting higher-order realized autocovariances appropriately as

$$
R K^{*}(\boldsymbol{y})=R C(\boldsymbol{y})+\sum_{h=1}^{n-1} k(h / H)\left\{\Gamma_{h}(\boldsymbol{y})+\Gamma_{-h}(\boldsymbol{y})\right\},
$$

for a bandwidth parameter $H=a n^{1 / 2}, a>0$, and, in particular, a non-stochastic kernel function, $k(h / H)$, carefully designed as

$$
k(z)=\mathbf{1}_{\{|z| \leq k\}}+\lambda(|z|-k) \mathbf{1}_{\{|z|>k\}}
$$

with $k=H^{-\gamma_{k}}, \gamma_{k} \in(0,1)$, a shrinking function of the bandwidth, and $\lambda(\cdot)$ a second-order smooth kernel function, satisfying some mild regularity conditions, an example being the Parzen kernel. The properties of these HAC-style estimators depend crucially on the kernel function, and by selecting $k(z)$ as in (12), the resulting class of flat-top realized kernels achieve optimal asymptotic properties in this setting, such as consistency, asymptotic unbiasedness, and mixed Gaussianity at the optimal rate of convergence, $n^{1 / 4}$, under mild assumptions on the MMS noise and (possibly random) sampling times. ${ }^{17}$ If optimally designed, the estimator is also efficient in a Cramér-Rao sense. As a result, it performs well in finite samples, even for sparse observations available at 1-5-minute frequencies. The estimator is implemented as suggested in Varneskov (2015b, Sections 4.2-4.3), with details provided in the supplementary appendix.

When intra-daily observations are only available for a certain part of a day, the trading window, and there is no recorded trading during weekends, holidays, etc., the estimates from the flat-top realized kernel may be supplemented with the squared close-to-open return since the preceding (trading) day. This approach essentially combines the estimates from $R C(\boldsymbol{x})$ and $R K^{*}(\boldsymbol{y})$ for data sampled at different frequencies to utilize all available observations. ${ }^{18}$

\subsection{A Simple Filtering Approach to Covariance Modeling}

A number of different procedures to construct one-step-ahead conditional expectations of the quadratic covariation matrix, $[\Delta \boldsymbol{x}]_{t+1}$, have been proposed in the literature, see, e.g., Andersen, Bollerslev, Christoffersen \& Diebold (2013, Section 3) for a recent review and many references. However, rather than searching for the best covariance model, the aim of this paper is to provide a baseline approach for dynamic implementation of the realized currency betas which is simple, of a non-parametric flavor, and easy to implement for quadratic covariation estimates with different degrees of measurement errors, such that it can accommodate within-period sampling at both daily- and intra-daily frequencies. In particular, the conditional procedure introduced here adapts the rolling window estimator originally

\footnotetext{
${ }^{17}$ The presence of an additive noise component slows down the best attainable rate of convergence for this estimation problem from $n^{1 / 2}$ to $n^{1 / 4}$, see Gloter \& Jacod $(2001 a, 2001 b)$.

${ }^{18}$ As explained in the supplementary appendix, the implementation procedure in Varneskov (2015b, Section 4.2) guarantees that the flat-top realized kernel is positive definite. Hence, a combination of the two estimators is also positive definite.
} 
proposed by Foster \& Nelson (1996) and Andreou \& Ghysels (2002) in the univariate case, and later extended to the multivariate case in Fleming, Kirby \& Ostdiek (2001), to the present setting. Thus, let $\boldsymbol{\Sigma}_{t}$ and $\hat{\boldsymbol{\Sigma}}_{t}$ be short-hand notation for the latent conditional quadratic covariation matrix and a generic estimator of this, respectively. Then the use of rolling window estimators implies the relation $\boldsymbol{\Sigma}_{t}=\sum_{i=1}^{\infty} \varpi_{t-i} \odot \hat{\boldsymbol{\Sigma}}_{t-i}$, where $\varpi_{t-i}$ is a symmetric $(C+1) \times(C+1)$ matrix of weights, and $\odot$ is the Hadamard product. ${ }^{19}$ As proved in Foster \& Nelson (1996, Theorem 5) under weak assumptions, the optimal weights are given by the exponential function $\varpi_{t-i}=\gamma \exp (-\gamma i) \iota \iota^{\prime}$ such that $\boldsymbol{\Sigma}_{t}$ may be recast as

$$
\boldsymbol{\Sigma}_{t}=\exp (-\gamma) \boldsymbol{\Sigma}_{t-1}+\gamma \exp (-\gamma) \hat{\boldsymbol{\Sigma}}_{t-1}
$$

In other words, the rolling window estimator may be thought of as an exponentially weighted GARCH model for the time series of quadratic covariation estimates $\hat{\boldsymbol{\Sigma}}_{t}$, whose rate of decay is determined by a single smoothing parameter $\gamma$. Despite being parsimoniously parameterized, this approach allows for persistent time-variation in $\boldsymbol{\Sigma}_{t}$, while implicitly reducing the impact of measurement errors in $\hat{\boldsymbol{\Sigma}}_{t}$. Furthermore, despite its simplistic structure, Varneskov \& Voev (2013) show that the forecasting performance of (13) is insignificantly different from that of more sophisticated multivariate Cholesky decomposed HAR and ARFIMA models when evaluated using a global minimum variance criterion for a portfolio of 10 stocks and various realized covariance measures. ${ }^{20}$ Finally, due to its simplistic structure, (13) may be easily implemented for different estimators of quadratic covariation and adapted to different investment horizons, which is important for real-time portfolio management.

\section{Data, Implementation, and Summary Statistics}

This section introduces the data set consisting of daily observations covering a long time span from January 1975 through August 2014. Furthermore, it contains details on the construction of quadratic covariation estimates for different investment horizons, along with the filtering approach used to implement the realized currency beta hedging strategy. Finally, novel evidence of time variation in optimal currency exposures is presented.

\subsection{A Long-Span Dataset of Daily Observations}

The empirical analysis is performed for a US investor who holds an equity portfolio and may use six different currencies from the G10 countries to hedge her foreign exchange rate exposure. In particular, these are the Canadian Dollar (CAD), the Swiss Franc (CHF), the Euro (EUR), the Great Britain Pound (GBP), the Japanese Yen (JPY), and the Swedish Krona (SEK). The daily foreign exchange rate series, as well as the remaining series described below, are obtained from the last spots quoted on

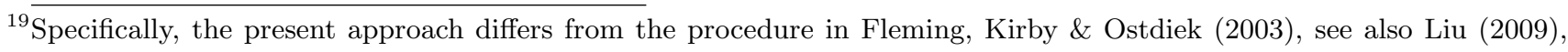
by replacing the outer product of returns, or the realized covariance estimator, with a generic quadratic covariation estimator, similarly to the study in Varneskov \& Voev (2013).

${ }^{20}$ Using a statistical loss function, on the other hand, Varneskov \& Voev (2013) find statistically significant gains from using multivariate Cholesky decomposed HAR and ARFIMA models over the multivariate GARCH model (13).
} 
Bloomberg on a given trading day, and all currency pairs are denominated as USD/foreign exchange rates. $^{21}$ Moreover, two different equity portfolios are considered. The first portfolio is the S\&P 500, whose currency exposure is determined implicitly through the international investments of its constituents. The second is an equally weighted portfolio in the stock markets of Canada, Germany, Great Britain, Japan, Sweden, Switzerland, and the US, using data from the DAX, NIKKEI 225, and S\&P 500 supplemented with data from MSCI Canada, Sweden, Switzerland, and the UK, all denominated in local currency. ${ }^{22}$ As such, the setup resembles that in Campbell et al. (2010), who, however, use monthly and quarterly observations, that is, there is no intra-period information in their hedging model. Furthermore, note that the SEK has replaced the Australian Dollar (AUD) as a hedging, or speculative, currency. A detailed motivation and discussion of this choice is deferred to Section 5.4.1 below. Finally, note that all return series are log-transformed.

Whereas Propositions 2 and 3 demonstrate that interest rate movements have no asymptotic impact on either the quadratic variation of the hedged portfolio log-returns or the realized currency betas, Proposition 1 shows that they need to be accounted for when gauging the performance of a hedging strategy in terms of its (log-)returns. However, it is difficult to find an appropriate daily risk-less interest rate series for each country, which cover the entire sample. Hence, to utilize as long a time span as possible for evaluation of the different hedging strategies considered, the interest rate differentials (relative to the US risk-less rate) are approximated by the CIP, $s_{c, t}-f_{c, t}=i_{c, t}-i_{0, t}$ with $\ln \left(1+I_{c, t}\right) \equiv$ $i_{c, t}=\int_{t}^{t+1} \lambda_{c, \tau} d \tau$ for $c=0, \ldots, C$, that is, using spot and forward exchange rate data, which may be supplemented with observations on the US risk-less rate. First, to get the forward rates, the spot exchange rates are combined with data on 3-month forward points, also obtained from Bloomberg, for a subsample period covering January 1989 through August 2014. Secondly, as a proxy for the US nominal risk-less rate, the 3-month ICE Libor is used. Lastly, all return series are carefully scaled to match the three investment horizons under consideration; one week, one month (four weeks), and one quarter (13 weeks).

(Table 1 - currency innovations summary statistics - around here)

Table 1 reports summary statistics for monthly log-changes in foreign exchange rates with respect to the USD over both the full sample and subsample, respectively monthly interest rate differentials relative to the US risk-less rate, and the resulting excess returns on foreign exchange rates. In particular, it displays the annualized mean, standard deviation, and Sharpe ratio, along with skewness and kurtosis statistics. ${ }^{23}$ A few noteworthy features emerge. First, the average changes in foreign

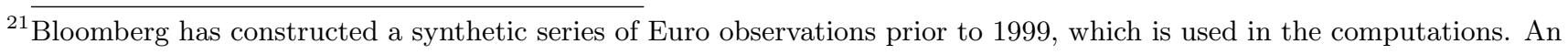
unreported robustness analysis, where the EUR log-return series is approximated using returns on the Deutsche Mark prior to 1999 and returns on the Euro after its introduction, has been performed, and the results are highly similar.

${ }^{22}$ The MSCI data for Canada, Sweden, Switzerland, and the UK, respectively, are used in place of the corresponding observations on the SPTSX 60, OMX Stockholm 30, SMI, and FTSE 100 indices since the latter series are not available prior to $1982,1986,1988$, and 1984, respectively.

${ }^{23}$ Note that since all summary statistics are computed directly from log-returns, there is no need to consider the Jensen's inequality correction in Proposition 1. The latter is only important when transferring log-returns back into gross return 
exchange rates differ quite substantially across countries in the full sample; the CHF and JPY have strongly appreciated against the USD, by 1.70 and 2.50 (annualized) percentage points per month, respectively, the CAD has appreciated, as well, but much less, and, finally, the EUR, GBP and SEK have all depreciated in value. These results, however, are time-dependent, and by considering the subsample, only, all currencies have appreciated against the USD, albeit by different amounts. Secondly, the results for interest rate differentials, which may be considered as the cost of currency investments, demonstrate that Switzerland and Japan have enjoyed the lowest risk-less interest rates, i.e., been the most expensive currency investments, which is consistent with both currencies being commonly used as funding sources for carry trade, see, e.g., Lustig, Roussanov \& Verdelhan (2011). Furthermore, the low standard deviation of the US risk-less rate and the excess interest rates illustrate that short-term interest rates are stable, in contrast to the much larger volatility on foreign exchange rates. Finally, the summary statistics for excess returns to foreign exchange rates demonstrate that an isolated position in a single currency is a poor investment strategy. The return is low, if positive, and volatility high. However, this does not preclude currencies from being effective hedging tools, e.g., for equity investments, or profitable when combined using different investment styles.

\subsection{Implementation of the Realized Currency Beta Strategies}

First, for a given investment horizon, a time series of realized covariation estimates is computed from daily returns and (10). From said estimates, three different realized currency beta hedging strategies are proposed; a semi-static hedge (SST), a fixed dynamic hedge (FDH), and an adaptive dynamic hedge $(\mathrm{ADH})$. Whereas all three strategies are implemented using a 5 -year initialization period, $T_{1}$, and an expanding window of observations, they differ with respect to their treatment of time variation in quadratic covariation. For specificity, at each time $t=T_{1}+1, \ldots, T$, the SST strategy is based on the in-sample (i.e., through $t-1$ ) unconditional average of the realized covariance estimates, while the FDH and ADH strategies are implemented using the filtering approach in (13), where $\boldsymbol{\Sigma}_{0}$ is estimated as the unconditional average from the first $T_{1}$ observations. The FDH uses a fixed smoothing parameter $\gamma=0.05$, and the ADH procedure selects the value of $\gamma \in(0, \infty)$ that minimizes in-sample equityreturn volatility for a realized currency beta hedged investor. ${ }^{24}$ The latter is a closed-form alternative to the numerical likelihood-based estimation procedures in, e.g., Fleming et al. (2003) and Varneskov \& Voev (2013), and eases the computational burden considerably. It is similar to the estimation procedure in Fleming et al. (2001), but differs by avoiding the use of forward-looking information, which would give the investor the benefit of hindsight. Both of these advantages are important for real-time investment decisions.

form. Moreover, and as seen by the general identity from Itô's lemma $R_{t}=r_{t}+[r]_{t} / 2$, the use of log-returns leads to a conservative assessment of the benefits from applying the proposed dynamic hedging strategies.

${ }^{24}$ The choice $\gamma=0.05$ may seem arbitrary. However, it corresponds well with both the average value of $\gamma$ selected using the $\mathrm{ADH}$ procedure for both a weekly- and monthly investment horizon, see Table 2, and is similar to the value determined for realized covariation measures in Fleming et al. (2003), Liu (2009), and Varneskov \& Voev (2013). Having a strategy with fixed $\gamma$ makes it feasible to disentangle the effects of estimation errors and time variation in $\gamma$ from the time variation in the quadratic covariation matrix, $\boldsymbol{\Sigma}_{t}$, and it serves as a robustness check. 
Two alternative hedging strategies are included as benchmarks. The first benchmark strategy is a full hedge, that is, $\boldsymbol{\beta}_{t}=\mathbf{0}$ for all $t$. The second benchmark strategy is a real-time implementation of the optimal hedging procedure analyzed, e.g., in Campbell et al. (2010), where, at each time $t=T_{1}+1, \ldots, T$, the covariance between the equity and foreign exchange returns is estimated by the (in-)sample covariance matrix using log-return series at the frequency matching the specific investment horizon, e.g., using weekly (monthly) returns for an investor rebalancing her portfolio at weekly (monthly) time intervals.

\subsection{Properties of Optimal Currency Exposure}

To facilitate understanding of the estimated foreign exchange rate exposures and to provide a preliminary gauge of the differences between alternative currency hedging procedures, Table 2 presents the means and standard deviations of the realized currency betas for SST, FDH, and ADH over the full sample when hedging an investment in the S\&P 500 using either one of the three selected rebalancing horizons. Moreover, the equivalent currency exposures from the unconditional approach in Campbell et al. (2010), labeled CMV, is included for comparison. ${ }^{25}$

(Table 2 - realized currency beta summary statistics - around here)

Consider a specific example from Table 2 to ease interpretation. The realized currency beta results for the FDH hedge and a monthly investment horizon demonstrate that it would have been optimal for an investor to hold large negative exposures to the CAD, EUR and SEK, on average, a large positive exposure to the CHF, and smaller positive exposures to the GBP and JPY. In particular, the reported average exposure to the CHF suggests that it is optimal to buy a portfolio of CHF-denominated bills worth 0.34 USD per USD invested in the S\&P 500. Similarly, the value -0.30 for CAD suggests that it is optimal to borrow 0.30 USD in CAD-denominated bills for every USD invested. The optimal exposure to the USD is implicitly determined by the zero net investment condition, $\boldsymbol{\beta}_{t}^{\prime} \boldsymbol{\iota}=0$. In the present case, this amounts to calculating the exposure as $-(-0.30+0.34-0.11+0.03+0.10-0.24)=0.18$ USD, which is equivalently to be placed in USD-denominated bills. When the equity portfolio contains explicit positions in foreign stock markets, the interpretation of the exposure-based hedging strategy is slightly more subtle, since it indicates how much to invest, or borrow, relative to the portfolio weight in a particular stock market. In general, however, the principle is the same.

There are noteworthy differences between the average currency exposures determined by the four hedging procedures. The CMV hedging strategy suggests holding much more underexposure to the CAD, much less exposure to the CHF, and less underexposure to the SEK, relative to the FDH and $\mathrm{ADH}$ strategies. Except for the large underexposure to the CAD, the same holds for the SST strategy. Furthermore, there are clear differences between the standard deviations of the optimal

\footnotetext{
${ }^{25} \mathrm{~A}$ similar table in the supplementary appendix reports qualitative identical, and quantitatively similar, results for the equally weighted equity portfolio (EW). Moreover, a figure corresponding to Figure 1 below, illustrating the time-variation in the optimal currency exposures for the EW portfolio, is also included in the supplementary appendix.
} 
currency exposures across strategies. The smallest standard deviations obtain for the SST strategy, followed by the CMV strategy, then the FDH and ADH strategies. As such, this ordering is not surprising. The SST exposures are based on the unconditional average from more precise measures of covariation than the CMV, realized covariance versus the outer-products of (demeaned) return vectors, whereas the larger variation in the currency exposures computed from the FDH and ADH strategies reflects, in part, that they allow the quadratic covariation matrix to vary over time. To flesh out the implications of the latter more clearly, Figure 1 illustrates the optimal currency exposures from 1980 through August 2014 using the CMV, SST, and ADH strategies for a weekly investment horizon. ${ }^{26}$

(Figure 1 - weekly exposure plots - around here)

Figure 1 reveals several interesting findings. First, the optimal currency exposures computed using the ADH strategy display pronounced time-variation, an important feature which neither the CMVnor the SST strategy captures. To highlight its importance, consider first the optimal exposure to the EUR as an example. In this case, if applying the CMV and SST strategies, the investor will be close to fully hedged $\left(\beta_{c, t} \simeq 0\right)$ throughout the sample. If applying a dynamic hedging strategy, however, it is optimal to hold positive exposures to the EUR in the first 4-5 years after its introduction in 1999, and large negative exposures during the most recent financial and subsequent European debt crises. In other words, the EUR switches from being a hedge $\left(\beta_{c, t}>0\right)$ to a speculative $\left(\beta_{c, t}<0\right)$ currency. Aside from this general pattern, a few key events stand out. For four weeks following the collapse of Lehman Brothers on September 15th, 2008, the optimal exposure switched from being large and negative to being postive, and subsequently dropping back to its original negative level. This spike, occurring within the course of one month, is substantial. In particular, the realized currency beta increases with $0.72-(-0.45)=1.17$ the week following the Lehman collapse, suggesting that it is optimal to re-position the currency exposure to the EUR in more than a one-to-one ratio with the portfolio value. Interestingly, the corresponding figure in the supplementary appendix, containing optimal exposures for the equal weighted portfolio, shows no dramatic fluctuations in currency exposure in reaction to the Lehman collapse, thereby highlighting two important features: One, there was a great need for outside investment opportunities for locally diversified US investors when their domestic stock market started to collapse. Two, and as mentioned earlier, the realized currency betas are portfolio specific and not a deep characteristic of the individual currencies. This is detailed in Section 7 . Note that since the dynamic hedging portfolio is zero net, the dramatic temporary increase in optimal exposure to the EUR is offset, or funded, by a large temporary short position the SEK. After the tumultuous fluctuations following the abnormal events during fall 2008, the optimal exposure to the EUR remains large and negative until July 2010, where it begins a gradual ascent toward full hedging. This ascent, however, comes to an abrupt end in August 2011, where the optimal exposure displays a sudden

${ }^{26}$ The FDH plot is similar to the one for $\mathrm{ADH}$ and is left out for ease of exposition. Note that the $x$-axis is slightly inaccurate as it is based on a count to 52 weeks per year and does not account for extra days due to leap years. Hence, toward the end of the sample, there are seven additional weeks relative to the count. However, it still serves the purpose of roughly tying time-variation in currency exposure to important economic events. 
large drop, contemporaneously with the sharp decline of several international stock markets in fear of contagion of the European sovereign debt crises in Italy and Spain.

The time-varying optimal exposures to the CHF comprise a second interesting example. From 1980 until the fall of 1998, the ADH strategy produces exposures that deviate from full hedging, albeit by smaller magnitudes. However, from 1999 and onwards, the large and positive realized currency betas illustrate the emergence of the CHF as an important hedging currency. The latter is best exemplified by the optimal exposures being particularly large over the time span from February 2006 until May 2010, thus covering the most recent financial crisis. These findings corroborate the perception of the CHF as a "safe haven currency", a notion detailed in Ranaldo \& Söderlind (2010). Interestingly, these positive exposures coincide exactly with a period where the CHF appreciated against major currencies, ending in May 2010 where, on the 18th, the Swiss National Bank (SNB) intervened to fight overvaluation of the CHF, leading to a $2 \%$ drop against the EUR. The subsequent decline of the realized currency betas, thus, matches well with the uncertainty surrounding valuation of the CHF and the SNB's eventual implementation of a minimum EUR exchange rate policy in September 2011. None of these features are captured by the semi-static CMV and SST strategies. Similar findings also relate to the realized currency betas for the JPY, which increase dramatically during the last seven years of the sample, starting in July 2007, illustrating its emergence as an important hedging currency. The gradual increase in optimal exposure for the JPY coincides well with its appreciation against all major currencies, except for the CHF, prior to the breakout of the most recent financial crisis.

As the realized currency betas solely utilize information from dynamic modeling of quadratic covariation matrices, it is striking to see such a strong coherence between their ascending patterns for the CHF and JPY prior to the breakout of the financial crisis and the contemporaneous appreciation of the two respective currencies against the remaining major currencies over the same time span. This suggests that the realized currency betas may carry useful information about local trends in currencies. Moreover, their ascent prior to the financial crisis may even suggest that large movements in realized currency betas could serve as early indicators of future financial turmoil. However, a deeper investigation of such postulates is warranted. For this covariance information to be useful in an international equity portfolio hedging context, the resulting large exposures need to provide protection, i.e., positive excess returns to the currency position in times when the equity portfolio performs poorly.

It is interesting to contrast the dynamic realized currency betas to the corresponding estimated unconditional exposures in prior work, e.g., those in Campbell et al. (2010, Table IV), who use monthly data from 1975 to 2005. A few stark contrasts are readily visible. Specifically, they find the EUR to be a hedge, the JPY to be a speculative currency, and the CHF and EUR to be substitutes, that is, the investor will not go wrong using either one of the two currencies as a hedge. If neglecting exposure movements from the last 9-10 years from Figure 1, a similar pattern emerges. However, exactly the last 9-10 years demonstrate the importance of a dynamic approach to currency hedging, since not only does the magnitude of optimal currency exposures change over time, the signs change as well. In this case, if the investor had used a static hedging procedure, she would be mislead into believing that a 
positive exposure to the EUR reduces the risk of an equity investment when, in fact, it amplifies it, and similarly for a negative exposure to the JPY. This may have detrimental effects for the performance of a hedged equity portfolio, in particular during financial turmoil.

Finally, it is worth noting that full hedging of equity investments, corresponding to $\boldsymbol{\beta}_{t}=\mathbf{0}$, is almost never optimal.

\section{Benefits from Dynamic Global Currency Hedging}

This section proceeds to demonstrate that not only do the estimated dynamic realized currency betas display interesting time-variation closely tied to important economic events, the resulting hedging strategies provide economic benefits to an investor above and beyond what is achieved by either fully hedged equity investments or semi-static optimal hedging strategies. The gains from dynamic currency hedging are shown from four different perspectives. First, risk-return results are provided for both the full sample and the subsample where interest rate differentials are accounted for in the return measurement. Second, the statistical significance of the volatility reductions are formally tested. Third, both the economic benefits to a risk-averse investor and transaction costs are assessed. Finally, robustness checks relating to prior work on currency hedging are carried out.

\subsection{A Gauge of the Risk-Return Benefits}

As an initial gauge of the benefits to currency hedging, Table 3 reports the annualized mean return, standard deviation, and Sharpe ratio, along with skewness and kurtosis statistics, for an equity investor, who implements one of five different hedging strategies to minimize the portfolio risk of foreign exchange rate exposure: Full hedging, CMV hedging, or one of the three realized currency beta strategies, SST, FDH, or ADH. The results are given for both the S\&P 500 and EW portfolios, using full sample data.

(Table 3 - full sample hedging statistics - around here)

Table 3 reveals several interesting findings. To highlight these, consider, first, the results for equity investments in the S\&P 500 with a monthly rebalancing horizon as an illustration. A clear pattern in terms of volatility reductions emerges. Relative to the fully hedged case, using either of the two semistatic hedging strategies, CMV and SST, reduces portfolio volatility with 140 basis points. Moreover, if the investor implements one of the two dynamic hedging strategies, FDH and ADH, she can achieve further volatility reductions in the 70-100 basis point range. From an economic perspective, these risk reductions are substantial, especially considering that they result from a zero net investment in foreign currencies. Second, note that each of the optimal strategies reduces the negative skewness in portfolio returns, and hence the downside risk properties of equity investments. Together with the volatility reductions, this shows that the proposed (dynamic) realized currency betas hedging procedures provide protection against negative returns and tail events. Even though interest rate 
differentials are not accounted for in the full sample, these will have a vanishingly small, if any, effect on the volatility reduction results, cf. Proposition 2. They may, however, impact the average return statistics as well as the estimated Sharpe ratios in Table 3, and one must therefore interpret these with caution. However, at least on the surface, the volatility reductions from optimal currency hedging seemingly come without sacrificing returns. As a result, the semi-static and dynamic hedging strategies improve the Sharpe ratio by roughly $10 \%$ and $30 \%$, respectively, relative to the fully hedged case. Again, this is a substantial economic gain from choosing currency exposure optimally.

The remaining parts of Table 3 demonstrate that the volatility reductions and (negative) skewness improvements from optimal currency hedging, especially those achieved by the dynamic strategies, pertain equally to the other rebalancing horizons, as well as to equity investments in the EW portfolio. The largest visible discrepancy in the results across rebalancing horizons for S\&P 500 investments is the deteriorating performance of the CMV strategy when using a quarterly re-hedging interval, for which, however, none of the three realized currency beta hedging strategies is affected. When comparing results across the two equity portfolios, the relative performance of different hedging strategies remains the same. The levels, however, for both returns and return-volatility statistics are lower for the EW portfolio than for the S\&P 500, reflecting, in part, abnormally high returns on US stocks over the course of the sample period, and equity diversification effects across international stock markets.

The corresponding subsample results, for which interest differentials have been taken into account when defining currency excess returns, are provided in Table 4. Note that these still reflect gross returns for the equity investments, i.e., the US risk-less rate has not been subtracted.

(Table 4 - subsample hedging statistics - around here)

In general, the subsample results confirm the favorable volatility properties arising from supplementing equity investments with optimal currency hedging strategies. When considering a monthly investment horizon, the semi-static hedges reduce the volatility of S\&P 500, respectively, EW investments relative to the fully hedged strategy by roughly 250-320 basis points for each, while the dynamic hedging strategies provide further gains of around 50-80 and 80-120 basis points for the respective portfolios. These results are generally consistent across rebalancing horizon. There are, however, differences for the relative return statistics across the two equity portfolios. The volatility reductions from applying the dynamic hedging strategies to S\&P 500 investments are accompanied by a slight increase in returns, even when accounting for interest rate differentials, whereas the same strategies lead to a small return loss for the EW investments. The respective Sharpe ratios for the two equity portfolios and different hedging strategies behave accordingly, but remain uniformly higher for optimal hedging strategies compared with full hedging. In general, the risk-return results demonstrate that investors in the S\&P 500 have received a better tradeoff than investors in the EW portfolio, and substantial improvements are visible for both portfolios when using, in particular, the proposed dynamic realized currency beta hedging strategies, which simultaneously reduce the negative portfolio skewness considerably. This demonstrates that the latter strategies generate such volatility and 
risk-return improvements while providing protection when stock markets decline. The timing of the payoff to the realized currency beta hedging strategies is elaborated upon and compared to traditional currency investment styles in Section 6.

In sum, optimal currency exposures are time-varying, and hedging strategies that are designed to utilize this time variation, such as those based on realized currency betas, substantially reduce portfolio volatility, relative not only to fully hedged equity investment, but also to equity investments hedged according to optimal static strategies previously considered in the literature. The volatility reductions are economically large, come completely without sacrificing returns for S\&P 500 investments, and lead to a better risk-return tradeoff for both equity portfolios, while simultaneously reducing downside portfolio risk.

\section{$5.2 \quad$ Significance testing}

This subsection eleborates on the results above by using the pairwise testing procedure of Ledoit \& Wolf (2011), building on their earlier work Ledoit \& Wolf (2008), to determine whether the volatility reductions from semi-static and dynamic hedging strategies are statistically significant. In particular, the procedure applies the delta method and HAC robust standard errors to calculate $t$-values for (log)volatility differences. Details are provided in the supplementary appendix. Since it is of interest to test whether all the optimal hedging procedures perform better than full hedging, as indicated by the point estimates in the previous subsection, the significance of the $p$-values are assessed against adjusted $p$-values using the conservative correction from Holm (1979) to avoid errors arising from multiple testing, see, e.g., Romano, Shaikh \& Wolf (2010) for a review.

Three separate hypotheses are of interest to the present analysis; whether the volatility reductions from the four optimal hedging procedures significantly exceed that from full hedging, and whether the volatility reductions from the dynamic hedging procedures exceed those from either the CMV or the SST hedging strategy. Table 5 and 6 report the results from the significance tests for hedged equity investments in the S\&P 500 and EW portfolio, respectively. In particular, they indicate whether the specific hypothesis has been rejected, and at which significance level, using Holm's adjusted $p$-values. The numerical $t$-statistics are deferred to the supplementary appendix for ease of exposition.

(Tables 5-6 - significance tests - around here)

The results in Tables 5 and 6 clearly demonstrate that optimal currency hedging strategies lead to significantly lower volatility in international equity investments than full hedging. For the CMV strategy, this corroborates the in-sample findings in Campbell et al. (2010, Table VII), improves the statistically insignificant evidence in Kroencke et al. (2014), and contrasts with the findings in Glen \& Jorion (1993) and de Roon et al. (2003). Furthermore, this constitutes the first statistical evidence in favor of the realized currency beta hedging approach, advanced in this paper. The volatility reductions for the dynamic hedging strategies are uniformly larger than the corresponding reductions from the semi-static strategies in Tables 3 and 4 . When testing the significance of this pattern for S\&P 500 
investments, the dynamic hedging strategies are observed to significantly outperform the CMV strategy for longer rebalancing horizons and the SST strategy for shorter rebalancing horizons. For the EW portfolio, the results are even stronger, as the dynamic hedging strategies yield significantly lower portfolio volatility than both semi-static strategies across all rebalancing horizons.

\subsection{Economic Benefits and Transaction Costs}

As emphasized by Marquering \& Verbeek (2004), Han (2006), and Della Corte et al. (2009), the Sharpe ratio may severely underestimate the performance of dynamic portfolio strategies. In particular, as the Sharpe ratio is computed using the full sample realized portfolio return and standard deviation, it may not adequately describe the conditional risk an investor faces at each point in time. Hence, and following Fleming et al. (2001), the economic value of a hedging strategy relative to a benchmark is assessed by determining the fee that may be subtracted from the hedged portfolio return corresponding to the proposed strategy each period, while still leaving average utility unchanged, compared to that achieved by investing according to the benchmark strategy. In other words, this fee equals the amount a risk-averse investor would be willing to pay in order to switch from the benchmark to the proposed strategy. Formally, as in Della Corte et al. (2009), let $Z_{t}^{p}=1+r_{t}^{p}$ and $Z_{t}^{b}=1+r_{t}^{b}$ be the payoffs to the proposal and the benchmark, respectively, then the switching fee $\Phi$ solves

$$
\sum_{t=T_{1}+1}^{T}\left\{\left(Z_{t}^{p}-\Phi\right)-\frac{\delta}{2(1+\delta)}\left(Z_{t}^{p}-\Phi\right)^{2}\right\}=\sum_{t=T_{1}+1}^{T}\left\{Z_{t}^{b}-\frac{\delta}{2(1+\delta)}\left(Z_{t}^{b}\right)^{2}\right\} .
$$

Specifically, (14) equates the average realized period-by-period utility across the proposed and benchmark strategies for an investor with quadratic preferences and relative risk-aversion indexed by the parameter $\delta$. In the empirical application, $\delta=8$ is fixed, see Fleming et al. (2001) and Della Corte et al. (2009) for detailed discussions of this preference specification. ${ }^{27}$

Table 7 reports the fee, $\Phi$, a risk-averse investor is willing to pay to switch, quoted in annualized basis points, for three different benchmarks (Full, CMV, and SST), a weekly, monthly and quarterly rebalancing horizon, and the two different equity portfolios, S\&P 500 and EW. Note that the results are only provided for the subsample from January 1989 through August 2014 where interest rate differentials are taken into account, to accurately measure the return effect.

(Table 7 - economic benefits - around here)

The economic value of applying a more sophisticated strategy than a fully hedge is clearly illustrated in Table 7. For example, for a monthly rebalancing horizon and the S\&P 500 portfolio, CMV and SST strategies provide gains that are worth around 300 annual basis points, whereas the dynamic FDH

\footnotetext{
${ }^{27}$ Fleming et al. (2001, 2003) fix $\delta=\{1,10\}$ and Della Corte et al. (2009) set $\delta=\{2,6\}$. A higher value of $\delta$, such as 10 , implies that the investor is willing to pay a higher fee for strategies that reduce portfolio volatility.
} 
and ADH strategies provide gains around 500 basis points. ${ }^{28}$ This difference between the semi-static and dynamic strategies suggests a fee of 200 basis points that the investor would be willing to pay to switch from the former to the latter. Indeed, fees ranging from 111-300 basis points for switching from the semi-static to the dynamic strategies are found throughout the table. Among the dynamic strategies, ADH is always worth more than FDH, whereas there is no similar ordering among the semistatic strategies, thus highlighting the incremental benefits to the investor of allowing the smoothing parameter $\gamma$ to change over time.

Of course, the choice of whether to implement a certain hedging strategy will also depend on transaction costs. More complicated strategies with more frequent trading will obviously be more costly to implement. Hence, it is important to compare the hedging gains to the associated costs. To do so, the transaction costs are assessed using a simple model, $\left|\tilde{\boldsymbol{\beta}}_{t}-\tilde{\boldsymbol{\beta}}_{t-1}\right| \times \mathcal{C}$, where $\mathcal{C}$ is an aggregate measure of transaction costs, intended to capture both trading fees, spreads, and any possible price impact. Inspired by, among others, Ramadorai (2008), who estimates the average transaction costs for major currencies to be around 3-4 basis points over the sample period 1994-2001, $\mathcal{C}$ is selected to be 10 basis points, thus providing a conservative assessment of the profitability of the strategies. ${ }^{29}$ The resulting transaction costs in annualized basis points are also provided in Table 7 . By construction, they are directly comparable to the reported fee $\Phi$. For example, for a monthly rebalancing horizon and the S\&P 500 portfolio, a risk-averse investor is willing to pay 190 basis points to switch from the CMV to the ADH strategy, at the expense of only $30.8-7.8=23.0$ additional basis points per year in net transaction costs. In general, across all combinations of rebalancing horizons and equity portfolios, the added economic benefits of switching from either a fully hedged or a semi-static hedging procedure outweigh the associated net transaction costs by a large margin.

All in all, the results demonstrate the economic- and statistical value of utilizing the underlying continuous-time movements in asset prices for discrete-time hedging to construct dynamic currency hedging strategies. The gains in volatility reductions from the latter compared with both full and semi-static hedging strategies offer substantial economic value to the investor, and are statistically significant.

\subsection{Additional Results and Robustness Checks}

This subsection summarizes the conclusions from two different robustness checks with respect to the set of hedging currencies, the detailed results of which may be found in the supplementary appendix.

\footnotetext{
${ }^{28} \overline{\text { Note that the switching fee estimates in Table } 7}$ are new to the currency hedging literature and, thus, provide further perspectives on the economic value a risk-averse investor receives from implementing also the existing (semi-)static optimal hedging procedures in Glen \& Jorion (1993), de Roon et al. (2003), and Campbell et al. (2010).

${ }^{29}$ Not only is $\mathcal{C}$ set more than twice as high as the estimated average transaction costs in Ramadorai (2008), Figure 1 further illustrates very clearly that the majority of currency trading for the dynamic ADH and FDH strategies, arising as a result of fluctuations in optimal exposures, occurs after 2000, when, among others, increased trading in and liquidity of foreign exchange rate contracts have reduced transaction costs compared to those estimated for the period 1994-2001, see, e.g., the estimated "effective costs" of currency trading in Mancini, Ranaldo \& Wrampelmeyer (2013).
} 


\subsubsection{Robustness to the SEK}

In the present paper, the SEK has replaced the AUD as a hedging, or speculative, currency, compared to the set of foreign exchange rates considered by Campbell et al. (2010). This is motivated, in part, by the latter, who find that the AUD and CAD are highly correlated and, to some extent, substitutes, cf. their Tables 2 and 5, which implies that the SEK may hedge some additional variation not covered by either one of the two. Hence, as a robustness check, the SEK is replaced with either the AUD or the New Zealand Dollar (NZD) in a hedging exercise similar to the one above. The results are similar, both in terms of economic magnitude of the volatility reductions and statistical significance. However, whereas the inclusion of the AUD in the set of hedging currencies generates slightly larger volatility reductions, these reductions are more expensive in terms of returns, amounting to lower economic gains for a risk-averse investor from using optimal currency hedging, compared with the results presented in Table 7. Finally, the gains from using the NZD fall between those for the AUD and SEK. These results partially explain why Campbell et al. (2010) often report smaller overall Sharpe ratios for the static optimal hedging strategy compared to full hedging in their supplementary appendix.

\subsubsection{Robustness to a Synthetic Carry Trade Currency}

Similarly to the design of the pseudo-dynamic hedging strategies in Glen \& Jorion (1993), de Roon et al. (2003), Campbell et al. (2010), and Kroencke et al. (2014), a synthetic carry trade currency is constructed using either equal or rank-based weighting of long and short positions in the six hedging currencies, based on the "pre-hedging" interest rate differentials relative to the US risk-less rate. In itself, the carry trade currency is shown to comprise a profitable trading strategy, corroborating the findings in, e.g., Lustig \& Verdelhan (2007), Lustig et al. (2011), and Menkhoff, Sarno, Schmeling \& Schrimpf (2012a). However, it is also demonstrated that its inclusion in the set of hedging currencies does not lead to any further volatility reductions, and its impact on the return of the equity portfolios is ambiguous in sign and small in magnitude. Unreported results show that the same conclusions arise when carry trade is formed based on ICE Libor or deposit rates in an even smaller subsample from 1994 through August 2014, or when adding a value synthetic currency to the set of hedging currencies.

Even though the inclusion of traditional foreign exchange rate trading strategies in the present setting yields no further volatility reductions, this does not mean that their inclusion in speculative asset allocation problems will not impact portfolio performance, see, e.g., Kroencke et al. (2014). Thus, although analysis of speculative asset allocation in a second step portfolio analysis is beyond the scope of the present paper, the performance of currency carry trade, momentum, and value strategies is used to provide further perspectives on the realized currency beta hedging strategies next.

\section{$6 \quad$ RCB and Traditional Currency Investment Styles}

The previous section demonstrates that the investor can achieve substantial gains in equity portfolio performance by supplementing the latter with a tactical foreign exchange rate overlay based on the 
proposed realized currency beta hedging procedure, specifically, the dynamic ADH approach. To synthesize and elaborate on these findings, this section relates the performance of the zero net currency portfolio from the ADH strategy to traditional currency investment styles, in particular, carry, momentum and value trading strategies. Details on the implementation of the latter are given in the supplementary appendix, where also summary statistics of their performance are provided for weekly, monthly, quarterly, and semi-annual rebalancing horizons. In general, carry trade performs best when implemented with equal weighted positions, whereas a rank-based weighting scheme is preferable for the two remaining strategies, momentum and value. Of course, since the present study "only" includes six foreign exchange rates against the USD, the analysis of these popular currency investment styles is more limited in scope than prior studies in the literature, such as Lustig et al. (2011, 2014), Menkhoff et al. (2012a, 2012b), and Asness et al. (2013). However, as the general, and relative, performance of the three currency trading strategies corresponds well with their findings, it may be used to provide new perspectives on the realized currency beta hedging procedure.

(Figure 2 - cumulative log-returns - around here)

Figure 2 displays the cumulative log-returns to the zero net currency portfolio generated by the dynamic ADH strategy when applied to the S\&P 500 index, together with the corresponding series for (fully hedged) S\&P 500 investments, as well as currency carry, momentum, and value trading strategies. They are all implemented using weekly rebalancing and the subsample from January 1989 through August 2014. Several interesting results are conveyed in Figure 2. First, the comparison against S\&P 500 investments corroborates the risk-reduction results in Tables 3-4 by showing that the ADH procedure delivers a currency trading strategy that is strongly negatively correlated with the equity portfolio, that is, it does, indeed, provide a hedge. Moreover, as it simultaneously produces a small positive average return, this generates the substantial economic benefits to the risk-averse investor shown in Table 7. The gains from hedging using the ADH procedure is clearly seen by its relative performance in the aftermath of the Lehman collapse. Over the four weeks following the latter, investors in the S\&P 500 loose about 32\%, whereas the ADH strategy delivers a $22 \%$ increase, thus dramatically reducing the portfolio losses for an optimally currency hedged investor.

Second, the dynamic currency hedging strategy is seen to be strongly negatively correlated with carry trade (correlation $\simeq-0.56$ ). The latter, in particular, performs well and generates positive returns until early 2008, when it not only ends its climb, it exhibits periodic losses, with the biggest decline occurring immediately after the Lehman collapse, where it yields a negative cumulative logreturn of $10 \%$ over the subsequent four weeks. Not only is this "crash risk" of carry trade consistent with prior findings in the literature, e.g., Brunnermeier et al. (2009), Burnside et al. (2011), and Menkhoff et al. (2012a), the negative correlation with the proposed dynamic realized currency beta hedging strategy as well as the use of the CHF and JPY as funding currencies (not reported) strongly suggest that carry traders are on the opposite end of the positive optimal exposures to said currencies in Figure 1, thus, at least partially, financing the good performance of the ADH procedure over these 
tumultuous four weeks. Moreover, the results suggest that the latter procedure could provide a good hedge for carry investments. These coherence results are striking, especially considering that the realized currency betas are asymptotically invariant to interest rate differentials (Proposition 3) and are implemented using only information about the (co)variances of currencies and the S\&P 500 index, not about local trends in the former.

Third, the correlations between the dynamic hedging strategy and momentum, respectively, value investments are mildly positive $(\simeq 0.19)$ and negative $(\simeq-0.29)$ for a weekly rebalancing horizon. Moreover, all three provide positive returns in the four weeks following the Lehman collapse, but the cumulative log-returns to momentum $(\simeq 16 \%)$ and value $(\simeq 7 \%)$ are both smaller than those generated by the use of the dynamic $\mathrm{ADH}$ procedure. Interestingly, the positive returns to momentum trading materialize two weeks after those to ADH. This suggests that while momentum trading relatively quickly picks up a shift in local trends, realized currency betas may, themselves, contain valuable information about expected returns to foreign exchange rates, indeed delivering instantaneous gains when the S\&P 500 declines dramatically. The supplementary appendix corroborates these findings by providing a "zoomed" version of Figure 2 that highlights the period spanning the financial and European debt crises, thus covering January 2008 through 2011. Moreover, the supplementary appendix documents that the magnitudes of the correlations between momentum, respectively, value investments and the dynamic hedging procedure remain modest for longer rebalancing horizons, and even switch sign for quarterly and semi-annual frequencies. Similarly, the correlations between momentum, respectively, value investments and the fully hedged S\&P 500 portfolio are modest and change sign depending on rebalancing horizon. In contrast, the unconditional correlations between the dynamic hedging strategy and the S\&P 500 index are strongly negative for all rebalancing horizons considered.

The negative correlation between carry trading and the dynamic realized currency beta based hedging strategy, as well as the mild correlations between the latter and momentum and value investments, suggest that there may be intriguing opportunities for combining the four different methods in designing tactical foreign exchange rate trading, as they each are based on and therefore convey different information. Whereas the traditional currency investment styles model local trends in currencies, the realized currency betas solely use information about their covariance with the equity portfolio. The proposed dynamic hedging procedure in this paper can, as a result, contribute with very favorable correlation properties and protection during market declines for equity portfolios and carry trade that is unparalleled by other existing currency investments styles, in particular, momentum and value. A detailed study of dynamic asset allocation across currency strategies is, however, left for further research.

\section{$7 \quad$ Realized Currency Beta Interpretation and Information}

The realized currency betas are specific to a given portfolio and describe the hedging potential from taking on currency exposure. Hence, they do not necessarily reflect deep characteristics of individual 
currencies, whose roles may change depending on the composition of the exogenous equity portfolio. As alluded to earlier, this is easily seen by comparing the dynamic optimal exposures to the EUR for the S\&P 500 equity portfolio in Figure 1 with the corresponding exposures for the EW portfolio, provided in the supplementary appendix. Whereas the study of dynamic currency hedging for the S\&P 500 and EW portfolios provides interesting results, as well as key insights into the interdependence between foreign exchange rates and equity investments, none of the two equity portfolios may adequately reflect a generic global benchmark. Hence, to complement the empirical hedging analysis in the previous sections and to provide general benchmark optimal currency exposures, the latter are computed by the dynamic ADH procedure using the MSCI World index as the equity portfolio. The resulting estimates, labelled the global equity realized currency betas, are provided in Figure $3{ }^{30}$ Moreover, the figure also depicts the difference between the global betas and the realized currency betas for the S\&P 500 portfolio, for direct comparability with Figure 1. The rebalancing horizon is weekly.

(Figure 3 - global realized currency betas - around here)

Figure 3 shows that the global equity realized currency betas, similarly, exhibit important timevariation, and it corroborates many of the findings in Figure 1: The role of the EUR switches from being a hedge after its introduction to becoming a speculative currency during the most recent financial and subsequent European debt crises, apart from a spike in demand following the Lehman collapse; the CHF and JPY emerge as important hedging currencies during the last 7-15 years of the sample; it is generally optimal to short the CAD and SEK; whereas relatively smaller fluctuations occur in the optimal exposure to the GBP. While these general patterns seem to describe the optimal currency exposures for the S\&P 500, EW, and MSCI World portfolios well, there are important differences between them. For example, it is optimal when hedging the S\&P 500 portfolio to have smaller exposure to the CHF than suggested by the global equity realized currency betas until 2007, when the trend reverses. A similar pattern, of opposite sign, is seen for the optimal exposures to the EUR. Moreover, it is optimal for investors in the S\&P 500 to have smaller short positions in the CAD and SEK as well as generally more exposure to the JPY than investors in the generic MSCI World portfolio.

The differences between realized currency betas across equity portfolios are time-varying and can be substantial, reflecting the importance of customizing the dynamic hedging strategy to the given equity portfolio. Hence, a comparison between portfolio specific optimal currency exposures and the global equity realized currency betas may informally be used to extract information about the underlying correlation properties of the assets in the equity portfolio. For example, the pronounced commonalities in optimal currency exposures across the three equity portfolios considered reflect similar correlation properties for the US stock market, the stock markets of the G10 countries in the EW portfolio, and the global equites in the MSCI World, which has a value skew toward developed stock markets. This

\footnotetext{
${ }^{30}$ Whereas the global equity realized currency betas provide benchmark dynamic optimal currency exposures, the resulting hedging performance is harder to evaluate, since it depends on the interest differentials for each of the countries in the index, cf. the first term in Proposition 1. Hence, the global betas are only used to study and provide generic properties of optimal exposures for the selected set of hedging currencies.
} 
is consistent with the evidence, e.g., in Longin \& Solnik (1995), Bekaert et al. (2009), Eun \& Lee (2010), and Christoffersen et al. (2012), who find that the correlations between international stock markets, especially for developed countries, have increased dramatically since the 1970's. However, the discrepancies in realized currency betas across the different equity portfolios also convey differences in the correlation properties of their respective underlying stock market investments. Not only does Figure 3 suggest that such differences are persistent over time, they may also change rapidly in periods of financial turmoil. For example, consider the time-varying differences in optimal exposures to the CAD. While it is consistently optimal to have less underexposure for investors in the S\&P 500 portfolio compared with equivalent investments in the MSCI World, two dramatic spikes stand out, namely, the weeks following Black Monday, October 19th, 1987, and the Lehman collapse. This clearly suggests that the optimal demand for diversification using currencies manifests itself differently depending on the composition of the equity portfolio, and that this demand is sensitive to the time-varying correlation properties of the underling equities, as well as to periods of financial turmoil.

These findings are elaborated upon in Figure 4, which contains the time-varying sum of absolute realized currency betas, not including the implicit position in USD, for the three equity portfolios considered, and a weekly rebalancing horizon.

(Figure 4 - sum of absolute realized currency betas - around here)

Speaking directly to the total optimal currency hedging demands for different equity portfolios, Figure 4 closely ties elevations and peaks to well-known periods of market turmoil. First, there is a spike in currency hedging demand following the Black Monday stock market crash. Interestingly, this only manifests itself for the EW and MSCI World portfolios, suggesting that non-US stock markets drive the increase in optimal short positions for the CAD in Figure 3. Second, there is an elevation of total currency demand in 1998, coinciding well with the Russian crisis. Similarly, large increases in total optimal exposures during mid-2002 until early 2003 occur contemporaneously with bearish stock markets brought about by events including the burst of the internet bubble. Lastly, the total optimal exposures remain large throughout the financial and European debt crises. Though there are some clear common patterns for the three equity portfolios, there are also equally clear differences in their response to important market events, and in the subsequent decay of optimal currency demands following elevations. For example, the total optimal currency hedging demand for the S\&P 500 portfolio hardly reacts to the 1998 Russian crisis, unlike the other two equity portfolios, and remains elevated much longer following the 2002-2003 bear market period and European debt crisis. This highlights the importance of choosing the realized currency betas specific to a given equity portfolio.

\section{Dynamic Global Currency Hedging using Intra-daily Data}

The proposed model for discrete-time hedging based on continuous-time movements in the underlying assets of interest is shown to generate substantial economic benefits for a risk-averse investor when 
implemented using daily data for weekly, monthly, and quarterly rebalancing horizons. These benefits are generated by the computation of dynamic realized currency betas, which accurately capture important time-variation in the optimal currency exposures for a given equity portfolio, especially during periods of financial turmoil, as explained in the previous section.

To provide further evidence on the proposed model, another data set, including both daily and intra-daily observations on equities and currencies, as well as daily observations on risk-less interest rates, is carefully collected, covering September 2005 through August 2014. This is subsequently used in a similar empirical hedging exercise to assess the economic value of actively utilizing the even richer information content in intra-daily data across assets, despite the observations being obtained from different exchanges, trading hours, and time zones.

\subsection{Construction of a Synchronized Data Set}

As a first step in constructing the new data set, which facilitates a hedging exercise based on either daily or higher frequency data to gauge the economic value of using an increased information set, intra-daily futures observations are obtained from Tick Data on the DAX, FTSE 100, Nikkei 225, and S\&P 500, together with the corresponding futures contracts on the CAD, CHF, EUR, GBP, and JPY exchange rates against the USD. These series are at most available on a one-minute frequency and cover different exchanges as well as different time zones and trading hours. The observations across contracts are generally non-synchronous, and it is important to suitably align them before proceeding with the analysis. Hence, the following four steps are proposed to generate a sequence of quadratic covariation estimates: (1) Identify the common trading days for all contracts. If at least one contract does not trade, the day is deemed a non-trading day; (2) Identify the common trading window for all trading days. If this set is empty, the day is deemed a non-trading day; (3) Synchronize the intra-daily observations from the common trading window using refresh time sampling (Martens 2004). Once synchronized, the equity portfolio log-returns are constructed by equally weighting log-returns on the four equity index futures; (4) If the number of synchronized observations is less than 39, quadratic covariation is estimated using the outer product of open-to-close returns. If the number is larger than 39 observations, quadratic covariation is estimated using flat-top realized kernels, as described in Section 3.1. The estimate in (4) is supplemented with the outer product of close-to-open log-returns from the preceding trading day to account for "overnight" comovements among assets.

The proposed four synchronization steps eliminate, among others, bank holidays and days where reduced trading hours for at least one contract trim the synchronized sample size to the extent where a high-quality estimate of quadratic covariation from the noise-robust flat-top realized kernels can no longer be guaranteed. However, since MMS noise, encompassing synchronization errors, and other market imperfections still cloak the underlying price processes, the properties of the realized covariance estimator in (10) will be distorted and, as a result, the open-to-close covariance estimator is selected to get an unbiased, albeit noisy, quadratic covariation estimate. ${ }^{31}$ Out of the 2274 common trading

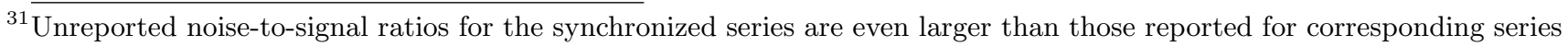


days in the sample, the constraint in step (4) binds three times. On average, however, there are 389 intra-daily observations available for quadratic covariation estimation. As described in the supplementary appendix, the flat-top realized kernels are guaranteed to be positive definite by applying an asymptotically negligible eigenvalue correction. The latter is needed on 21 trading days.

The generated sequence of intra-daily data based quadratic covariation estimates is supplemented with daily log-returns from spot observations of the same equity indices and exchange rates as above, similarly to the data described in Section 4.1, as well as risk-less interest rates, which are approximated using 3-month deposit rates for the CAD, EUR, GBP, JPY, and USD, along with the 3-month ICE Libor for the CHF. These short-term risk-less interest rate series are all directly available for the subsample under consideration from September 2005 through August 2014 and, thus, replace the CIP approximations from Section 4.1.32 The daily observations are carefully constructed to match the same trading days as the intra-daily quadratic covariation estimates and subsequently scaled to match two different investment horizons; one week and one month (four weeks). The quarterly investment horizon is dropped from this empirical exercise since a relatively short time span does not leave enough out-of-sample observations to guarantee a reasonable statistical precision of the results.

Finally, the implementation of ADH strategies using either daily or intra-daily quadratic covariation estimates as input, ADH-D and ADH-HF, respectively, is adapted slightly here to accommodate having a subsample with a shorter time span, and dominated by highly tumultuous market events such as the most recent financial crisis and subsequent European debt crisis. First, the initialization period, $T_{1}$, is selected to be one year, leaving a very interesting period, covering September 2006 through August 2014, for out-of-sample evaluation of the proposed hedging strategies. Moreover, as, e.g., Bollerslev \& Wright (2000) find that one year of data often suffices to estimate the persistence of highfrequency measures of volatility with reasonable precision, this leaves enough observations to estimate the smoothing parameter, $\gamma$. Secondly, the objective function in the closed-form estimation procedure is changed from an in-sample global minimum volatility criterion, using the portfolio returns for a realized currency beta hedged investor, to the corresponding in-sample mean absolute deviation. The latter is a more robust measure of variation in the presence of outliers, initially promoted by Hampel (1974), and its use greatly stabilizes the parameter estimates, in particular when including the period surrounding the most recent financial crisis for estimation.

\subsection{Hedging Benefits from Intra-daily Data}

Initially, the differences between the realized currency betas computed from the ADH-D and ADH-HF strategies are gauged in Figure 5, which provides scatter plots of the respective exposures for each hedging currency, including the implied exposures to the USD, as well as fitted linear regressions.

of individual S\&P 500 stocks, e.g., in Varneskov (2015b), clearly reflecting the challenge of extracting information from observations across different markets, time zones, and trading hours.

${ }^{32}$ The use of intra-daily data prior to September 2005 almost always leaves fewer than 39 observations for quadratic covariation estimation due to a short common trading window across assets, markets, and time zones. The period is, thus, excluded to get an accurate assessment of the economic value of using intra-daily instead of daily data. 
(Figure 5 - scatter plots - around here)

Figure 5 shows that there is systematic coherence between the estimated realized currency betas for the CAD, JPY, and USD, whereas the remaining currency exposures display pronounced dispersion. The fitted linear regressions corroborate the visual evidence. If the two strategies generate the same hedging implications, the constant $\left(b_{0}\right)$ and slope $\left(b_{1}\right)$ from the currency-wise regressions should equal 0 and 1 , respectively. However, this is clearly rejected for all currencies. For example, the estimates $b_{0}>0$ and $b_{1} \approx 0.65<1$ for the JPY suggest that the ADH-HF strategy, on average, generates larger and less volatile exposures to the JPY than ADH-D, and, despite exhibiting coherence, there is clear dispersion in the estimated hedging demands. If considering the CHF, another hedging currency, the differences between two methods are much more pronounced. In particular, whereas the estimated regression constant $b_{0}>0$, again, suggests that the ADH-HF strategy, on average, produces larger hedging demands, the estimated slope $b_{1} \approx 0.15$ implies that there is little, if any, linear coherence between the two methods. Moreover, a decomposition of the slope into standard deviations of 0.31 and 0.69 for the ADH-HF and ADH-D based exposures, respectively, as well as a correlation of 0.32 between the two suggests that the low coherence arises from two sources: (1) The exposures from the ADH-D strategy being more variable over time; (2) the differences between the information content in intra-daily and daily data revealing strikingly different comovement patterns between the log-returns on the CHF and the equity portfolio, as reflected by the low correlation. To elaborate on (2), the ADHD strategy is seen to generate exposures below -1 all of nine times out of 402 weekly observations, whereas the realized currency betas from ADH-HF never go below -0.39. It is even more remarkable that these extreme negative exposures for the ADH-D strategy are always of opposite sign compared with the corresponding exposures from $\mathrm{ADH}-\mathrm{HF}$, the methods thereby fundamentally disagreeing on whether the $\mathrm{CHF}$ is a speculative or hedging currency. Similar comments apply to the remaining currencies.

Clearly, the use of intra-daily data to estimate quadratic covariation generates different hedging implications than using daily observations. However, whether this improves portfolio performance remains to be determined. Hence, Table 8 compares the performance of an equal weighted equity portfolio for an investor implementing the ADH-HF strategy instead of either the ADH-D strategy or a full hedge, similarly to the long-span analysis in Sections 5.1-5.3.

(Table 8 - HF hedging statistics - around here)

The advantages of using the dynamic realized currency beta hedging framework are clearly demonstrated in Table 8. Both ADH procedures deliver statistically significant volatility reductions of around 550 and 480 basis points for a weekly, respectively, monthly rebalancing horizon. However, whereas the ADH-D strategy achieves said reductions without loss of returns, the ADH-HF strategy even produces additional return gains of around 120 and 200 basis points, respectively. As a result, the hedging strategy delivers substantial improvements in portfolio performance over an eight year time span characterized by several episodes of financial turmoil. Its Sharpe ratios are more than $100 \%$ 
larger than those achieved by fully hedged equity investments, and around 60-75\% larger than those of an investor using daily data to implement the realized currency beta hedging framework. These results clearly testify to the need for having a diversified portfolio during extreme market events, and highlight that foreign exchange rates can be important investment vehicles if their trading is carried out systematically and dynamically. In particular, Table 8 shows that a risk averse investor is willing to pay more than 800 annual basis points to make a switch from fully hedging equity investments to a dynamic hedging strategy based on intra-daily quadratic covariation measures, and 150-200 basis points to adopt the latter strategy instead of one based on corresponding daily covariation measures.

The substantial economic value of the ADH-HF strategy speaks directly to the usefulness of the proposed model for currency hedging. The use of intra-daily data provides a better approximation to the underlying continuous-time movements in the assets of interest between portfolio rebalances, thereby facilitating more accurate extraction of information about important comovements between the equity portfolio and hedging currencies. Moreover, it improves the finite sample approximations of the asymptotic results in Propositions 2-3 by reducing the impact of, among others, risk-less interest rate effects. Combining these insights with the results from the long-span analysis using daily data, this provide overwhelming evidence in favor of the realized currency beta hedging framework as a systematic currency trading methodology that not only significantly improves portfolio performance, it furthermore corrects previous caveats in the literature. As a result, it constitutes a powerful alternative to traditional currency trading strategies such as carry trade, momentum, and value investments, as examined in Section 6 for the long-span data set using the ADH-D procedure, but it differs fundamentally by being designed to improve the performance of an already existing portfolio, and by using (co)variation information, only.

\section{A Jump-Diffusion Generalization}

The theoretical results in Propositions 1-3 rely on the design of, and assumptions for, the vector price system in (3)-(5). Whereas the latter presents a considerable generalization compared to the extant literature on currency hedging, it is widely recognized in the literature on high-frequency volatility estimation that the underlying price processes may contain discontinuous as well as a diffusive risk sources. To analyze the role of discontinuities, or jumps, suppose the within-period movements in equities and currencies instead of (3) and (4), respectively, obey more general jump-diffusions

$$
\begin{aligned}
& d P_{c, \tau} / P_{c, \tau-}=\mu_{c, \tau} d \tau+\sigma_{c, \tau} d W_{c, \tau}+\left(\exp \left(d_{c, \tau}\right)-1\right) d N_{c, \tau} \quad \text { and } \\
& d S_{c, \tau} / S_{c, \tau-}=\alpha_{c, \tau} d \tau+\varphi_{c, \tau} d Y_{c, \tau}+\left(\exp \left(k_{c, \tau}\right)-1\right) d M_{c, \tau}, \quad c=0, \ldots, C,
\end{aligned}
$$

where $\left(N_{c, \tau}, M_{c, \tau}\right)$ is a pair of finite activity Poisson processes and $\left(d_{c, \tau}, k_{c, \tau}\right)$ captures the corresponding stochastic jump sizes through an exponential transformation. The risk-less bond prices are still assumed to obey (5). To formally analyze hedging implications for the generalized system (5), (15) 
and (16), the following additional structure is imposed on the jump components:

Assumption 3. Suppose the jump components of the intra-period price system in (15) and (16) satisfy the following conditions for all $c \in\{0, \ldots, C\}$ :

(i) $\left(N_{c, \tau}, M_{c, \tau}\right)$ are $\mathcal{F}_{\tau}$-adapted finite activity Poisson processes that are independent of the corresponding Brownian motions $\left(W_{c, \tau}, Y_{c, \tau}\right)$;

(ii) The sequence of jump sizes, $\left(d_{c, \tau}, k_{c, \tau}\right)$, is $\mathcal{F}_{\tau}$-measurable, $\min _{s=1, \ldots, N_{\tau}}\left|d_{c, s}\right| \in(0, \infty)$ and, similarly, $\min _{s=1, \ldots, M_{\tau}}\left|k_{c, s}\right| \in(0, \infty)$. Moreover, the sequence of jump sizes is independent of both $\left(d N_{c, \tau}, d M_{c, \tau}\right)$ and the Brownian increments $\left(d W_{c, \tau}, d Y_{c, \tau}\right)$;

(iii) $\left[d N_{c, \tau}, d M_{c, \tau}\right]=d L_{c, \tau}$ where $L_{c, \tau}$ is an $\mathcal{F}_{\tau}$-adapted finite acitivity Poisson process.

While conditions $(i)$-(ii) of Assumption 3 are standard in the literature on jump-robust estimation of integrated diffusive variation and jump testing, ${ }^{33}$ condition (iii) specifies a general stochastic process for the co-jump intensity between the country-wise equity index and exchange rate against the USD, thus accommodating large simultaneous discontinuous movements in the two series. The latter captures, among others, rare economic events such as those on September 6th, 2011, where the Swiss National Bank announced that it would intervene to fight "overvaluation" of the Swiss Franc, resulting in a $4 \%$ increase of the SMI, as well as a $9 \%$ decrease of the CHF against the USD. ${ }^{34}$ Note that condition (iii) specifically parameterizes such co-jumps for a given country $c$ since they appear in the asymptotic representation result below. In general, however, the price system in (15) and (16), as well as Assumption 3, accommodates co-jumps across multiple equity indices and currencies.

Before proceeding, let $\tilde{d}_{c, \tau}=\left(\exp \left(d_{c, \tau}\right)-1\right), \tilde{k}_{c, \tau}=\left(\exp \left(k_{c, \tau}\right)-1\right)$, and stack the respective jump sizes as $(C+1) \times 1$ vectors $\tilde{\boldsymbol{d}}_{\tau}=\left(\tilde{d}_{0, \tau}, \ldots, \tilde{d}_{C, \tau}\right)^{\prime}$ and $\tilde{\boldsymbol{k}}_{\tau}=\left(\tilde{k}_{0, \tau}, \ldots, \tilde{k}_{C, \tau}\right)^{\prime}$. Similarly, let $\boldsymbol{X}_{\tau}=$ $\left(X_{0, \tau}, \ldots, X_{C, \tau}\right)^{\prime}$ for $X=\{d, k, N, M, L\}$ and use - for the inner product of two equally dimensioned vectors. Then the following proposition provides a representation result for the log-return of a currency hedged portfolio, assuming the generalized within-period price system in (5), (15) and (16):

Proposition 4. Suppose that the instantaneous currency hedged return may be described using the within-period price system in (5), (15) and (16) as

$$
\frac{d V_{\tau}}{V_{\tau-}}=\sum_{c=0}^{C} w_{c, t} \frac{d\left(P_{c, \tau} S_{c, \tau}\right)}{P_{c, \tau-} S_{c, \tau-}}+\sum_{c=0}^{C} \theta_{c, t} \frac{d\left(B_{0, \tau} / B_{c, \tau}\right)}{\left(B_{0, \tau} / B_{c, \tau}\right)}-\sum_{c=0}^{C} \theta_{c, t} \frac{d S_{c, \tau}}{S_{c, \tau-}}
$$

similarly to (6), and that Assumptions 1-3 hold, then

$$
d v_{t}=\boldsymbol{w}_{t}^{\prime}\left(d \boldsymbol{p}_{\tau}+\boldsymbol{\lambda}_{0, \tau} d \tau-\boldsymbol{\lambda}_{\tau} d \tau\right)+\boldsymbol{\beta}_{t}^{\prime}\left(d \boldsymbol{s}_{\tau}-\boldsymbol{\lambda}_{0, \tau} d \tau+\boldsymbol{\lambda}_{\tau} d \tau\right)+\Sigma_{\tau}^{h} d \tau+J_{\tau}^{e}+o_{p}(d \tau)
$$

\footnotetext{
${ }^{33} \overline{\text { See, among others, Barndorff-Nielsen \& Shephard }}$ (2004b, 2007), Huang \& Tauchen (2005), Aït-Sahalia \& Jacod (2012), Andersen, Dobrev \& Schaumburg (2012), Varneskov (2015a), and many references therein.

${ }^{34}$ Source: "Swiss National Bank acts to weaken strong franc", BBC News, Business Section, September 6th, 2011.
} 
where $\Sigma_{\tau}^{h}$ is the same Jensen's inequality correction as in Proposition 1, and where

$$
J_{\tau}^{e}=\tilde{J}_{\tau}^{e}+\sum_{\ell=2}^{\infty}(-1)^{(\ell-1)} \frac{J_{\tau}^{\ell}}{\ell} \quad \text { with } \tilde{J}_{\tau}^{e}=\boldsymbol{w}_{t}^{\prime}\left(\boldsymbol{d}_{\tau}^{e} \cdot d \boldsymbol{N}_{\tau}\right)+\boldsymbol{\beta}_{t}^{\prime}\left(\boldsymbol{k}_{\tau}^{e} \cdot d \boldsymbol{N}_{\tau}\right)+\boldsymbol{w}_{t}^{\prime}\left(\tilde{\boldsymbol{d}}_{\tau} \cdot \tilde{\boldsymbol{k}}_{\tau} \cdot d \boldsymbol{L}_{\tau}\right)
$$

as well as $J_{\tau}=\tilde{J}_{\tau}^{e}+\boldsymbol{w}_{t}^{\prime}\left(\boldsymbol{d}_{\tau} \cdot d \boldsymbol{N}_{\tau}\right)+\boldsymbol{\beta}_{t}^{\prime}\left(\boldsymbol{k}_{\tau} \cdot d \boldsymbol{N}_{\tau}\right), \boldsymbol{d}_{\tau}^{e}=\tilde{\boldsymbol{d}}_{\tau}-\boldsymbol{d}_{\tau}$, and $\boldsymbol{k}_{\tau}^{e}=\tilde{\boldsymbol{k}}_{\tau}-\boldsymbol{k}_{\tau}$, collects the Itô approximation errors arising from the discontinuous parts of the jump-diffusions (15) and (16).

Proof. See Appendix A.4.

Proposition 4 generalizes Proposition 1 in two important ways. First, the hedged portfolio return is allowed to exhibit jumps through $d \boldsymbol{p}_{\tau}$ and $d \boldsymbol{s}_{\tau}$, whose collective impact depends not only on the size and frequency of the jumps themselves, but also on the vector of portfolio weights and currency exposures, respectively. Secondly, it includes $J_{e}$, which contains the Itô approximation errors due to jumps in (15) and (16). Moreover, Proposition 4 demonstrates that such jump approximation errors decompose into first- and higher-order terms, which are each comprised of economically intuitive error sources. As an example, consider the first-order error, $\tilde{J}_{\tau}^{e}$, which consists of three separate components. The first two of these components measure the distance between the jumps $\left(\tilde{\boldsymbol{d}}_{\tau}, \tilde{\boldsymbol{k}}_{\tau}\right)$ and their approximation under logarithmic transformation $\left(\boldsymbol{d}_{\tau}, \boldsymbol{k}_{\tau}\right)$, weighted by either the portfolio weights or currency exposures. The last component, on the other hand, quantifies the impact of country-wise equity index and currency co-jumps, whose presence follows immediately from the first term in $d V_{\tau} / V_{\tau-}$.

Whether the presence of (co-)jumps renders changes to the design of the currency hedging procedures necessary or not depends crucially on the magnitude of the jump approximation errors, $J_{\tau}^{e}$. If the latter is small $\left(J_{\tau}^{e} \simeq 0\right)$ for all $\tau$ or is dwarfed in magnitude by the realized jumps, that is, $\left|J_{\tau}^{e}\right| \ll\left\|\left(\boldsymbol{d}_{\tau}, \boldsymbol{k}_{\tau}\right)\right\|$, then to a fairly good approximation,

$$
\left[d v_{\tau}\right]_{t+1} \simeq\left[\boldsymbol{w}_{t}^{\prime} d \boldsymbol{p}_{\tau}+\boldsymbol{\beta}_{t}^{\prime} d \boldsymbol{s}_{\tau}\right]_{t+1} \quad \text { and } \quad \tilde{\boldsymbol{\beta}}_{t}^{*} \simeq-\mathbb{E}_{t}\left[\left[d \tilde{\boldsymbol{s}}_{\tau}\right]_{t+1}\right]^{-1} \mathbb{E}_{t}\left[\left[\boldsymbol{w}_{t}^{\prime} d \boldsymbol{p}_{\tau}, d \tilde{\boldsymbol{s}}_{\tau}\right]_{t+1}\right]
$$

remains valid. This implies that the proposed hedging procedures may be carried as described in the previous sections, with the subtle change in interpretation that realized currency betas are, in this case, based on estimates of the total quadratic covariation matrices, thereby including variation from both diffusive and jump risk sources. ${ }^{35}$ The model in (15)-(16) and Proposition 4 accommodates a much generalized array of market events and movements, such as the described co-jump in the SMI and the USD-CHF exchange rate on September 6th, 2011. The practical implication in the present dynamic hedging setting is that the choice of exposures based on realized currency betas works with and without jumps, i.e., investors can afford to be agnostic regarding their existence.

If, on the other hand, the jump approximation errors are large, then $\left[d v_{\tau}\right]_{t+1}$ will deviate substantially from $\left[\boldsymbol{w}_{t}^{\prime} d \boldsymbol{p}_{\tau}+\boldsymbol{\beta}_{t}^{\prime} d \boldsymbol{s}_{\tau}\right]_{t+1}$, resulting in a wedge between the realized currency betas and optimal

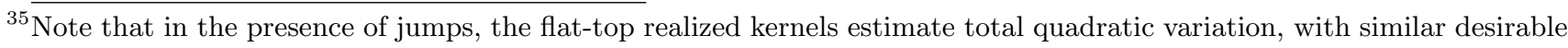
asymptotic properties as those described in Section 3.1, see Varneskov (2015a, Theorem 2).
} 
currency exposures, which, in this case, also depend on the path-wise properties of $J_{\tau}^{e}$. Hence, as an informal assessment of the magnitude of the approximation errors, consider again the abnormal events on September 6th, 2011, as an example. Here, a co-jump of $4 \%$ in the SMI and $9 \%$ in the USD-CHF exchange rate result in logarithmic approximation errors of size $\exp (0.04)-1-0.04 \simeq 0.0008$ and $\exp (0.09)-1-0.09 \simeq 0.0042$, respectively, as well as a co-jump error of size 0.0036 , all of which are at least an order of magnitude smaller than the jumps themselves. More generally, Figure 6 shows the logarithmic jump approximation errors and co-jump errors for various jump sizes. In all cases, the results corroborate the conclusions from the Swiss example; the errors are, at least, an order of magnitude smaller than the jumps themselves. This illustrates that the realized currency betas provide a fairly good approximation to the optimal currency exposures under general conditions on the within-period equity price- and currency movements. Finally, it is important to note that the substantial economic benefits achieved by the dynamic realized currency beta hedging procedure, as illustrated by the empirical analysis, is in itself evidence in favor of the approximation (17).

(Figure 6 - approximation errors - around here)

\section{Conclusion}

This paper proposes a model for discrete-time hedging based on continuous-time movements in portfolio and exchange rate returns. The vector of optimal currency exposures are shown to be negative realized regression coefficients computed from a one-period conditional expectation of the intra-period quadratic covariation matrix for portfolio and foreign exchange rate returns, which are labelled the realized currency betas. The theoretical model, hence, facilitates the design of dynamic hedging strategies that depend exclusively on the evolution of the intra-period quadratic covariation matrix. This implies that interest differentials have no asymptotic impact on optimal currency hedging demands, and that an investor should sample observations as frequently as possible in fixed time intervals between portfolio rebalances to improve the accuracy of the quadratic covariation estimates. Both implications contrast with prior theoretical results in the extant currency hedging literature, which assume that assets are observed at the same frequency as that at which the portfolio is being rebalanced. Moreover, since the proposed strategies only use information from the covariance between foreign exchange rate and portfolio returns, not about local trends in the former, they are distinctly different from traditional currency investment styles, such as carry, momentum, and value investments.

The realized currency beta hedging strategies are implemented using modern, yet simple, nonparametric techniques to accurately measure and dynamically model historical quadratic covariance matrices. Methodologically, this procedure addresses two important caveats in the literature: (1) The lack of dynamic modeling of optimal currency exposures, except when tied to slowly varying conditioning variables, such as past interest rate differentials; and (2) the use of forward-looking information when estimating the optimal exposures, thus providing the investor with the benefit of 
hindsight. Addressing both caveats is important for accurate assessments of intertemporal currency hedging demands and real-time investment decisions.

In an extensive empirical analysis, the use of the new hedging strategies, based on realized currency betas, produces novel results: $(i)$ The optimal currency exposures display substantial time-variation, especially over the period covering January 1999 through August 2014 where, e.g., the Euro switches from being a hedge after its introduction to becoming a speculative currency during the most recent financial crisis and subsequent European debt crisis. Moreover, such time-variation can be tied to important economic events. (ii) The proposed dynamic hedging strategies produce statistically significant, as well economically substantial, volatility reductions for international equity portfolios, compared to either fully hedging currency exposure, or using existing static optimal hedging produces, without sacrificing returns. (iii) Using a long time span data set of daily observations, a risk averse investor is shown willing to pay 400-500 basis points to switch from a fully hedged static position to the proposed dynamic hedging strategies. Moreover, the investor is willing to pay 170-300 basis points to make the switch from optimal static to optimal dynamic hedging strategies. (iv) The addition of a synthetic carry trade currency to the set of hedging currencies, mimicking prior pseudo-dynamic strategies in the global currency hedging literature, produces no further gain in portfolio performance. (v) The proposed dynamic realized currency beta investment strategy is unconditionally negatively correlated with carry trade, and only modestly correlated with momentum and value investments. Interestingly, the empirical analysis strongly suggest that carry traders, at least partially, fund the strong performance of the proposed dynamic strategy during the most recent global financial crisis of 2008. (vi) The realized currency betas display similar dynamic patterns for different equity portfolios. However, they also exhibit persistent differences in levels, as well as different temporary elevations and spikes in response to economic events. (vii) Using a carefully collected data set of intra-daily observations, covering September 2005 through August 2014, to construct even more accurate estimates of quadratic covariation, leads to the design of dynamic hedging strategies worth more than 800 basis points relative to being fully hedged, and worth 150-200 basis points more than a corresponding dynamic hedging strategy based on daily data.

Finally, the proposed hedging model is generalized by allowing for discontinuities, or jumps, in the within-period processes of interest, specifically, in equities and currencies, allowing the model to encompass and explain a much broader array of market events. This is shown to generate similar hedging implications, realized currency betas now being computed from estimates of total quadratic covariation matrices, thereby including variation from both diffusive and jump risk sources. Thus, the generalization has implications for the proposed dynamic hedging strategies in terms of interpretation, but this does not alter the evidence in their favor. 


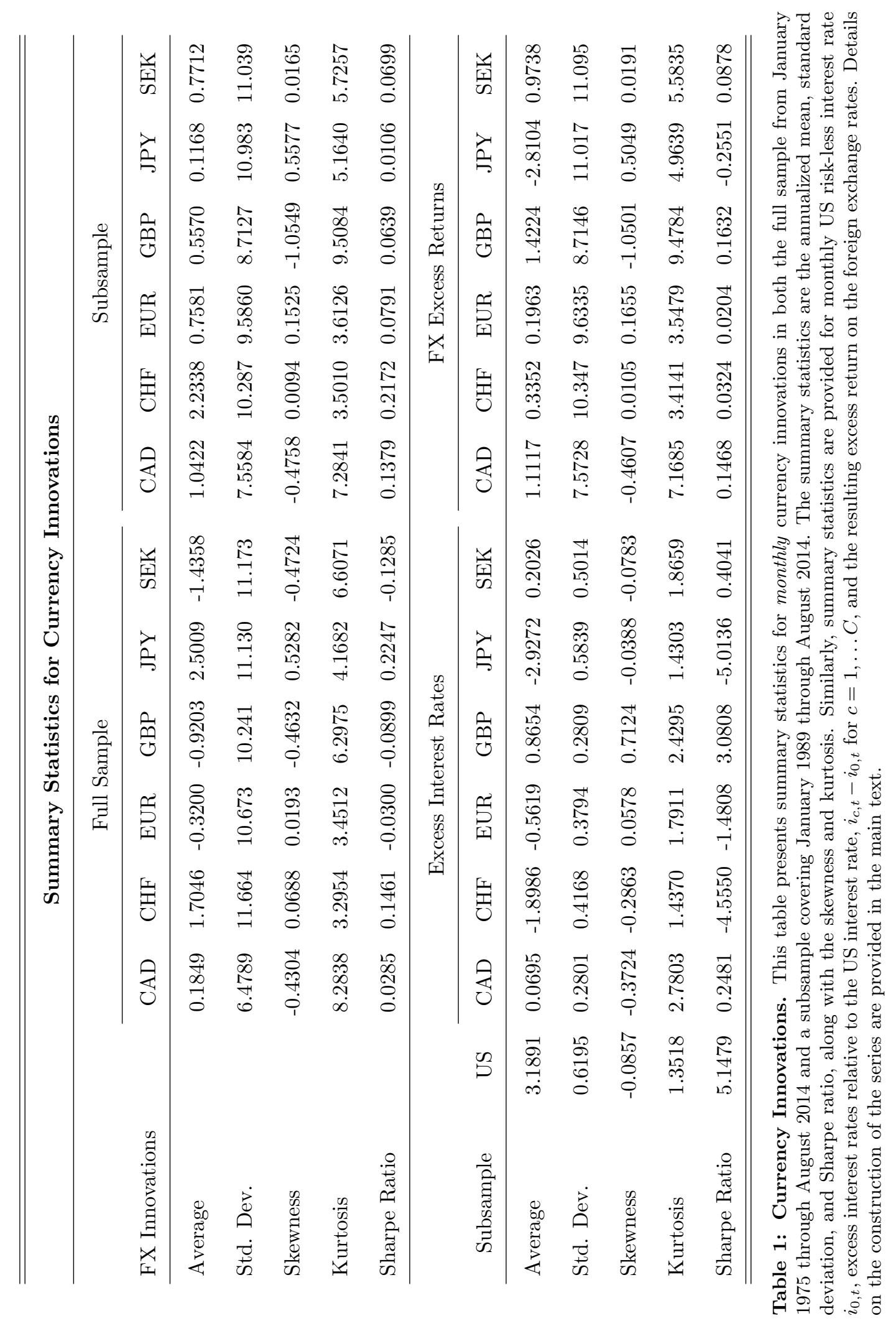




\begin{tabular}{|c|c|c|c|c|c|c|c|c|}
\hline \multicolumn{9}{|c|}{ Summary Statistics for Realized Currency Betas - S\&P 500} \\
\hline & \multicolumn{4}{|c|}{ Average } & \multicolumn{4}{|c|}{ Standard Deviation } \\
\hline & $\mathrm{CMV}$ & $\mathrm{SST}$ & FDH & $\mathrm{ADH}$ & CMV & SST & FDH & $\mathrm{ADH}$ \\
\hline \multicolumn{9}{|c|}{ Weekly } \\
\hline$\gamma$ & - & - & 0.0500 & 0.0221 & - & - & 0.0000 & 0.0142 \\
\hline CAD & -0.4791 & -0.3360 & -0.2870 & -0.2934 & 0.1276 & 0.0630 & 0.3664 & 0.2788 \\
\hline CHF & 0.1084 & 0.1026 & 0.3446 & 0.3231 & 0.1105 & 0.1133 & 0.4681 & 0.4492 \\
\hline EUR & -0.1067 & -0.0129 & -0.1448 & -0.1460 & 0.0548 & 0.0576 & 0.6343 & 0.5468 \\
\hline GBP & -0.0067 & 0.0046 & 0.0337 & 0.0317 & 0.0370 & 0.0250 & 0.1997 & 0.1444 \\
\hline JPY & 0.0328 & 0.0315 & 0.0797 & 0.0844 & 0.0487 & 0.0567 & 0.2802 & 0.2534 \\
\hline SEK & -0.0519 & -0.0726 & -0.2330 & -0.2093 & 0.1375 & 0.0924 & 0.3945 & 0.2896 \\
\hline \multicolumn{9}{|c|}{ Monthly } \\
\hline$\gamma$ & - & - & 0.0500 & 0.0790 & - & - & 0.0000 & 0.0896 \\
\hline CAD & -0.7641 & -0.3413 & -0.2964 & -0.2854 & 0.1087 & 0.0623 & 0.2143 & 0.2501 \\
\hline CHF & 0.1050 & 0.1035 & 0.3375 & 0.3554 & 0.2115 & 0.1134 & 0.4032 & 0.4190 \\
\hline EUR & 0.0746 & -0.0105 & -0.1132 & -0.0266 & 0.2048 & 0.0574 & 0.4342 & 0.3876 \\
\hline GBP & -0.0537 & 0.0044 & 0.0283 & 0.0279 & 0.1144 & 0.0247 & 0.0870 & 0.1420 \\
\hline JPY & -0.0529 & 0.0308 & 0.0999 & 0.0838 & 0.0570 & 0.0566 & 0.2339 & 0.2157 \\
\hline SEK & -0.0908 & -0.0716 & -0.2392 & -0.2848 & 0.1912 & 0.0927 & 0.2554 & 0.3240 \\
\hline \multicolumn{9}{|c|}{ Quarterly } \\
\hline$\gamma$ & - & - & 0.0500 & 0.1350 & - & - & 0.0000 & 0.1116 \\
\hline CAD & -0.9027 & -0.3470 & -0.3177 & -0.3145 & 0.1973 & 0.0619 & 0.1174 & 0.2061 \\
\hline $\mathrm{CHF}$ & 0.0291 & 0.1042 & 0.2854 & 0.3652 & 0.1604 & 0.1143 & 0.3303 & 0.3844 \\
\hline EUR & 0.3999 & -0.0101 & -0.0676 & -0.1406 & 0.1680 & 0.0570 & 0.2488 & 0.4563 \\
\hline GBP & 0.1719 & 0.0024 & 0.0103 & 0.0322 & 0.1642 & 0.0245 & 0.0499 & 0.0980 \\
\hline JPY & -0.2568 & 0.0284 & 0.0787 & 0.1150 & 0.1293 & 0.0565 & 0.1719 & 0.2426 \\
\hline SEK & -0.4366 & -0.0672 & -0.1813 & -0.2235 & 0.2312 & 0.0941 & 0.2041 & 0.2639 \\
\hline
\end{tabular}

Table 2: Realized currency betas. This table provides the average and standard deviation of the realized currency betas for all hedging currencies, four different implementations of the realized currency betas, and three different rebalancing horizons; one week, one month (four weeks), and one quarter (13 weeks). The statistics, in particular, are provided for the S\&P 500 equity portfolio over the full sample from 1975 through August 2014. CMV is a real-time implementation of the procedure in Campbell et al. (2010), SST is the semi-static implementation using quadratic covariation estimates, whereas both the FDH and ADH are dynamic strategies, which differ with respect to estimation of the smoothing parameter. See Section 4.2 for details. Note that the first five years of data are used for initialization. Finally, similar statistics are provided for the smoothing parameter, $\gamma$. 


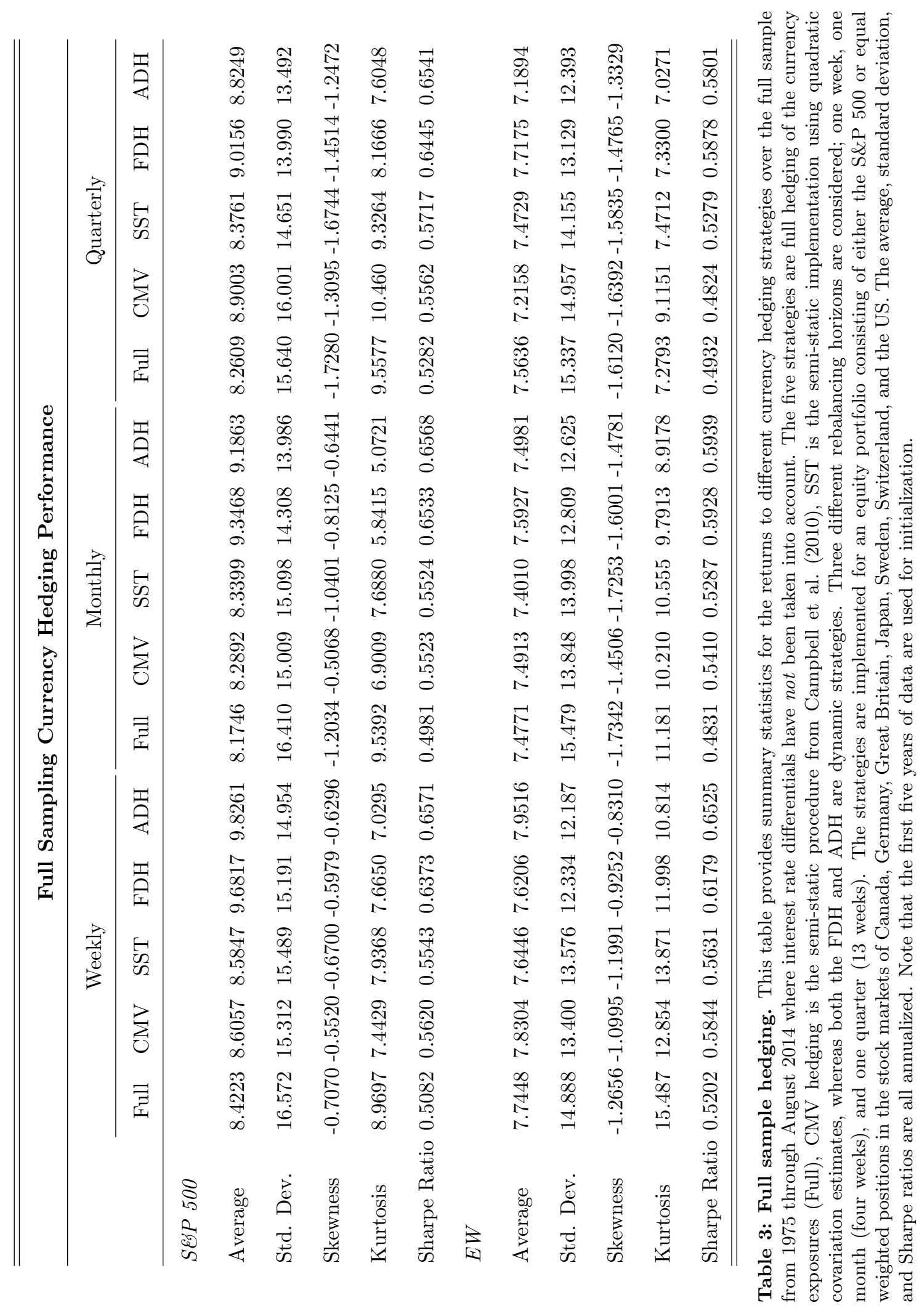




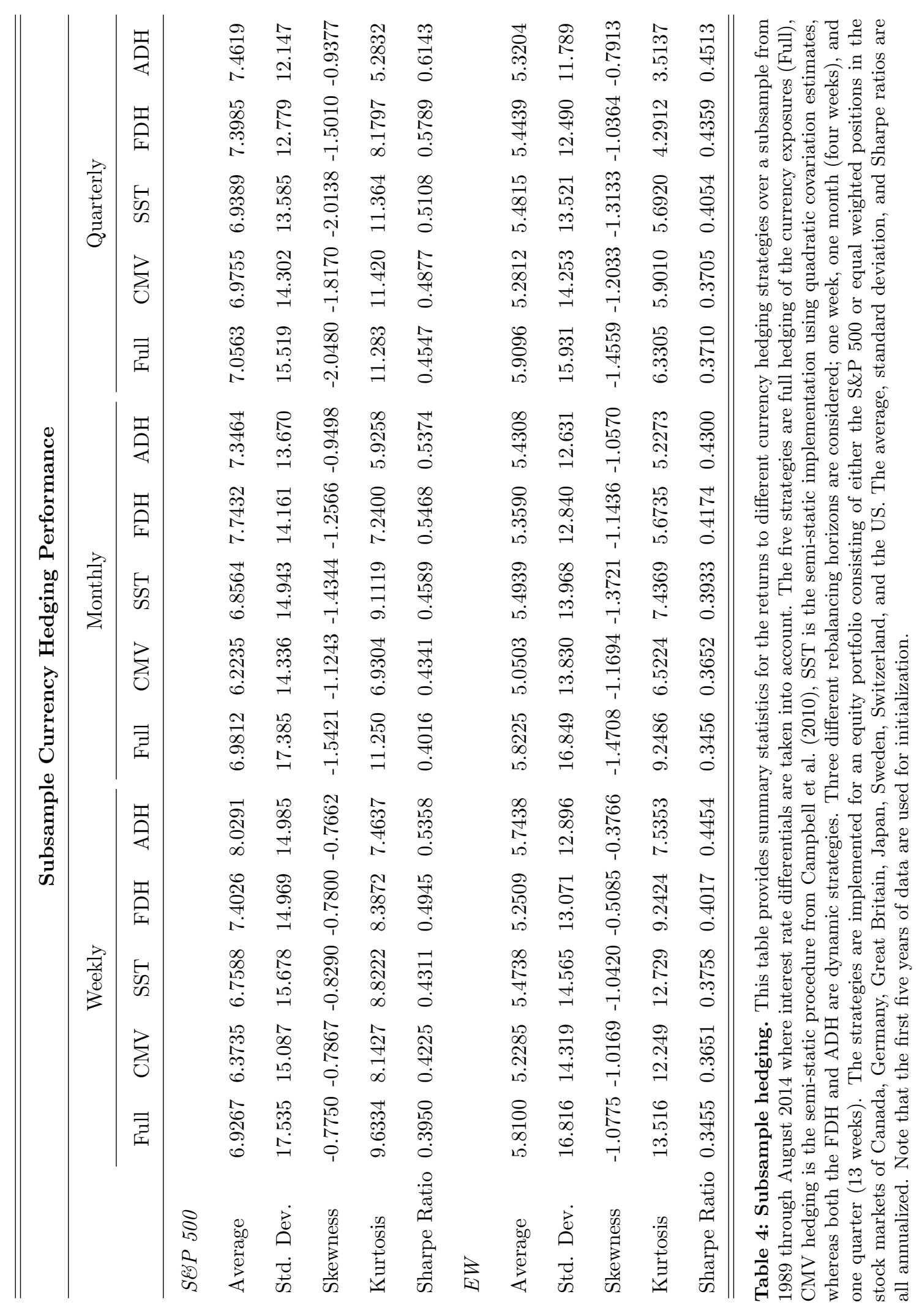




\begin{tabular}{|c|c|c|c|c|c|c|}
\hline & \multicolumn{6}{|c|}{ Significance Tests for S\&P 500 Investments } \\
\hline & \multicolumn{3}{|c|}{ Full Sample } & \multicolumn{3}{|c|}{ Subsample } \\
\hline & Weekly & Monthly & Quarterly & Weekly & Monthly & Quarterly \\
\hline \multicolumn{7}{|c|}{ vs Full hedging } \\
\hline CMV & $* * *$ & $* *$ & $\mathrm{~N}$ & $* * *$ & $* * *$ & $* * *$ \\
\hline SST & $* * *$ & $* * *$ & $* *$ & $* * *$ & $* * *$ & $* * *$ \\
\hline $\mathrm{FDH}$ & $* * *$ & $* * *$ & $* *$ & $* * *$ & $* * *$ & $* * *$ \\
\hline $\mathrm{ADH}$ & $* * *$ & $* * *$ & * & $* * *$ & $* * *$ & $* *$ \\
\hline \multicolumn{7}{|c|}{ vs $C M V$ hedging } \\
\hline FDH & $\mathrm{N}$ & $\mathrm{N}$ & $* *$ & $\mathrm{~N}$ & $\mathrm{~N}$ & $* *$ \\
\hline $\mathrm{ADH}$ & $\mathrm{N}$ & $*$ & $* *$ & $\mathrm{~N}$ & $\mathrm{~N}$ & $* *$ \\
\hline \multicolumn{7}{|c|}{ vs SST hedging } \\
\hline FDH & $\mathrm{N}$ & $*$ & $\mathrm{~N}$ & $\mathrm{~N}$ & $\mathrm{~N}$ & $\mathrm{~N}$ \\
\hline $\mathrm{ADH}$ & $*$ & $* *$ & $\mathrm{~N}$ & $\mathrm{~N}$ & $*$ & $\mathrm{~N}$ \\
\hline
\end{tabular}

Table 5: Significance tests for S\&P 500. This table shows the conclusions from pairwise significance tests for three separate hypothesis. The pairwise tests are based on the procedure of Ledoit \& Wolf $(2008,2011)$, which is described in the supplementary appendix. The three null hypotheses of interest are: (1) whether the volatility from a fully hedged portfolio return is the same as that from the four optimal hedging procedures; $(2)$ whether the volatility from a CMV hedged portfolio return is the same as that from the two dynamic hedging procedures; (3) same as (2) but with SST in place of CMV. Since multiple testing is performed, all estimated $p$-values are assessed against the adjusted $p$-values using the conservative correction by Holm $(1979) .\left(^{*}\right),(* *),(* * *)$ denote rejection at a $90 \%, 95 \%, 99 \%$ significance level in favor of the alternative hedging strategy. If rejection is in favor of the benchmark hedging strategy, all asterisks are preceded by a -1 . If the test does not reject, " $N$ " is reported. 


\begin{tabular}{|c|c|c|c|c|c|c|}
\hline \multicolumn{7}{|c|}{ Significance Tests for EW Investments } \\
\hline & \multicolumn{3}{|c|}{ Full Sample } & \multicolumn{3}{|c|}{ Subsample } \\
\hline & Weekly & Monthly & Quarterly & Weekly & Monthly & Quarterly \\
\hline \multicolumn{7}{|c|}{ vs Full hedging } \\
\hline CMV & $* * *$ & $* * *$ & $\mathrm{~N}$ & $* * *$ & $* * *$ & $* *$ \\
\hline SST & $* * *$ & $* * *$ & $* * *$ & $* * *$ & $* * *$ & $* * *$ \\
\hline $\mathrm{FDH}$ & $* * *$ & $* * *$ & $* *$ & $* * *$ & $* * *$ & $* * *$ \\
\hline $\mathrm{ADH}$ & $* * *$ & $* * *$ & $* *$ & $* * *$ & $* * *$ & $* * *$ \\
\hline \multicolumn{7}{|c|}{ vs $C M V$ hedging } \\
\hline FDH & $* * *$ & $* * *$ & $* *$ & $* * *$ & * & $* *$ \\
\hline $\mathrm{ADH}$ & $* * *$ & $* * *$ & $* *$ & $* * *$ & $* *$ & $*$ \\
\hline \multicolumn{7}{|c|}{ vs SST hedging } \\
\hline $\mathrm{FDH}$ & $* * *$ & $* * *$ & $* *$ & $* * *$ & $* *$ & $*$ \\
\hline $\mathrm{ADH}$ & $* * *$ & $* * *$ & $* *$ & $* * *$ & $* *$ & $\mathrm{~N}$ \\
\hline
\end{tabular}

Table 6: Significance tests for EW. This table shows the conclusions from pairwise significance tests for three separate hypothesis. The pairwise tests are based on the procedure of Ledoit \& Wolf $(2008,2011)$, which is described in the supplementary appendix. The three null hypotheses of interest are: (1) whether the volatility from a fully hedged portfolio return is the same as that from the four optimal hedging procedures; (2) whether the volatility from a CMV hedged portfolio return is the same as that from the two dynamic hedging procedures; (3) same as (2) but with SST in place of CMV. Since multiple testing is performed, all estimated $p$-values are assessed against the adjusted $p$-values using the conservative correction by Holm $(1979) .(*),(* *),(* * *)$ denote rejection at a $90 \%, 95 \%$, $99 \%$ significance level in favor of the alternative hedging strategy. If rejection is in favor of the benchmark hedging strategy, all asterisks are preceded by a -1 . If the test does not reject, "N" is reported. 


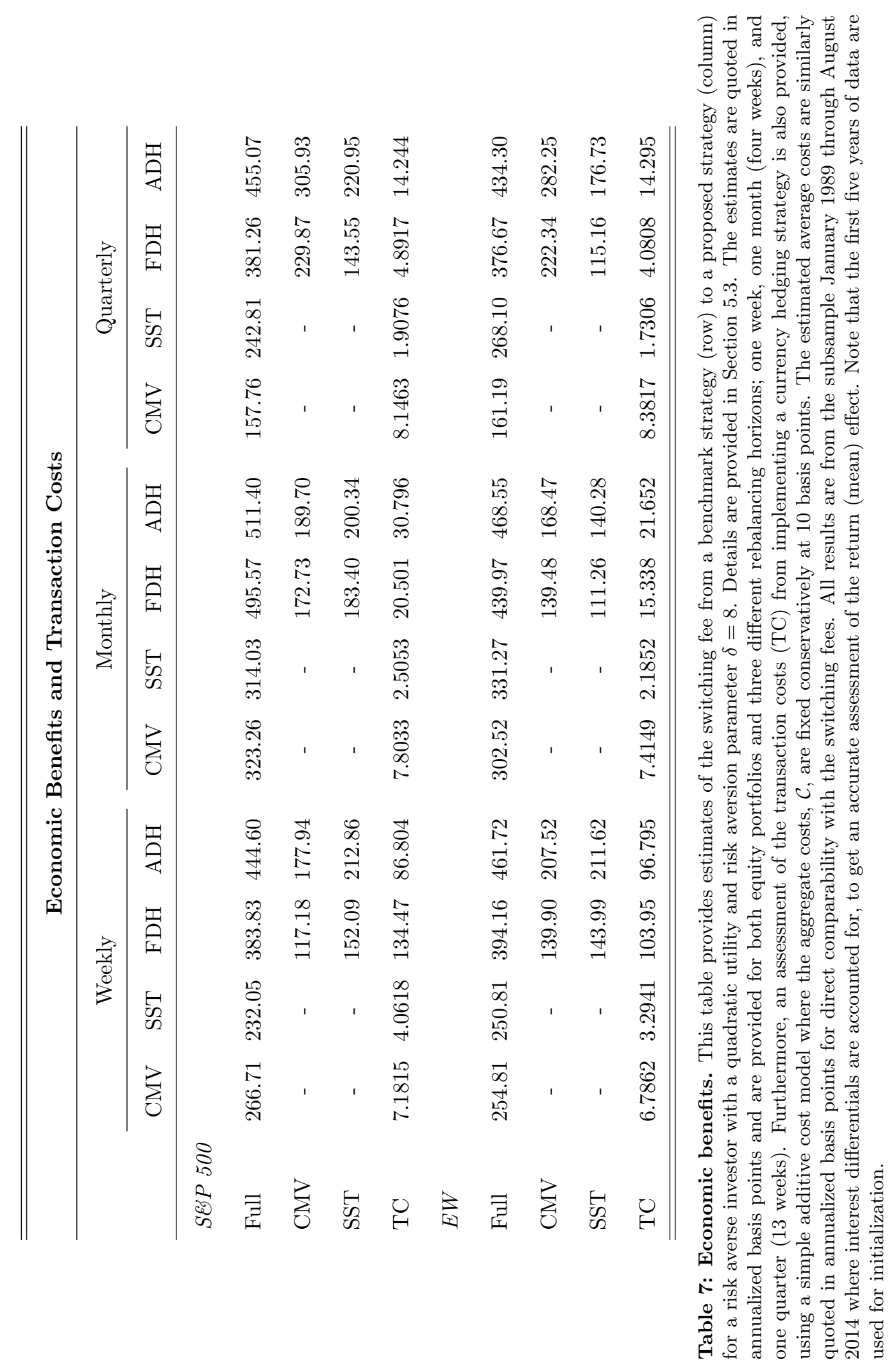




\begin{tabular}{|c|c|c|c|c|c|c|}
\hline \multicolumn{7}{|c|}{ Global Currency Hedging with High-Frequency Data } \\
\hline & \multicolumn{3}{|c|}{ Weekly } & \multicolumn{3}{|c|}{ Monthly } \\
\hline & Full & ADH-D & ADH-HF & Full & ADH-D & ADH-HF \\
\hline \multicolumn{7}{|c|}{ A: Summary Statistics } \\
\hline $\bar{\gamma}$ & - & 0.2355 & 0.2612 & - & 0.1440 & 0.9835 \\
\hline$\sigma(\gamma)$ & - & 0.1229 & 0.2095 & - & 0.1544 & 0.6730 \\
\hline Average & 2.3160 & 2.2569 & 3.4933 & 2.3182 & 2.4629 & 4.4456 \\
\hline Std. Dev. & 19.066 & 13.519 & 13.275 & 19.334 & 14.507 & 14.506 \\
\hline Skewness & -0.4717 & -0.5984 & -1.2978 & -1.1660 & -1.4094 & -1.9597 \\
\hline Kurtosis & 5.7438 & 6.7493 & 9.1200 & 5.0225 & 6.2322 & 10.044 \\
\hline Sharpe Ratio & 0.1215 & 0.1670 & 0.2632 & 0.1199 & 0.1698 & 0.3065 \\
\hline \multicolumn{7}{|c|}{ B: Volatility Testing } \\
\hline vs Full & - & $* * *$ & $* * *$ & - & $* * *$ & $* * *$ \\
\hline \multicolumn{7}{|c|}{ C: Economic Benefits } \\
\hline Full & - & 715.67 & 865.26 & - & 664.13 & 862.47 \\
\hline $\mathrm{ADH}-\mathrm{D}$ & - & - & 149.88 & - & - & 198.35 \\
\hline $\mathrm{TC}$ & - & 472.57 & 223.85 & - & 53.129 & 94.043 \\
\hline
\end{tabular}

Table 8: Hedging with high-frequency data. This table provides summary statistics and comparisons of a fully hedged portfolio (Full) with the proposed dynamic ADH strateg,y using both daily and intra-daily data (ADH-D and $\mathrm{ADH}-\mathrm{HF}$ ) to construct the quadratic covariation for an equity portfolio with equal weighted positions in Germany, Great Britain, Japan, and the US, using a subsample from September 2005 through August 2014. Panel A provides the average and standard deviation of the estimated smoothing parameters, $\bar{\gamma}$ and $\sigma(\gamma)$, the annualized average, standard deviation, and Sharpe ratio of the hedging strategies, as well as skewness and kurtosis statistics. Panel B provides significance tests for whether the dynamic hedging strategies have significantly lower volatility than a fully hedged portfolio. See Section 5.2 and Tables 5-6 for details. Panel C assesses the economic benefits of switching from a fully hedged benchmark portfolio (row) to a dynamic hedging strategy (column), the benefits of switching from a dynamic strategy based on daily data to one based on intra-daily, and the transaction costs associated with dynamic hedging. All are quoted in annualized basis points, see Section 5.3 and Table 7 for details. 
CAD Exposures

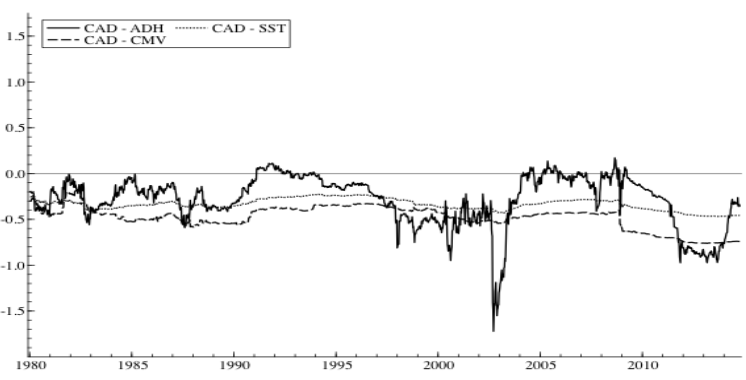

EUR Exposures

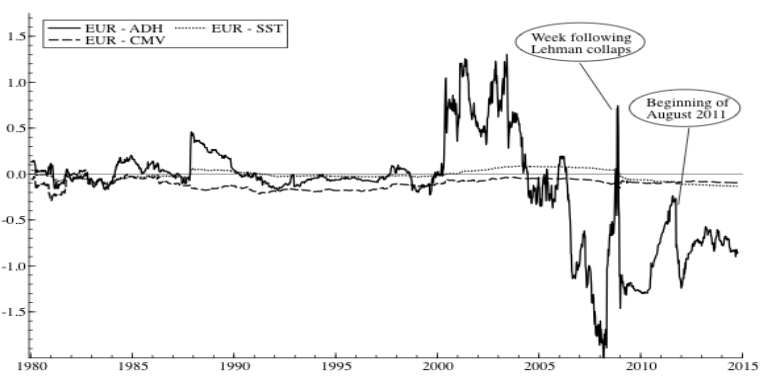

JPY Exposures

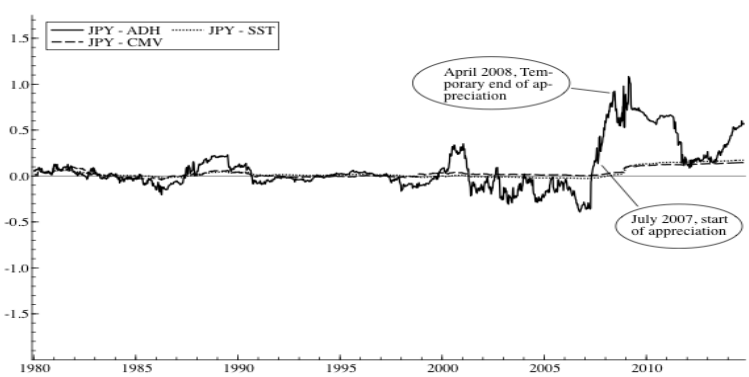

CHF Exposures

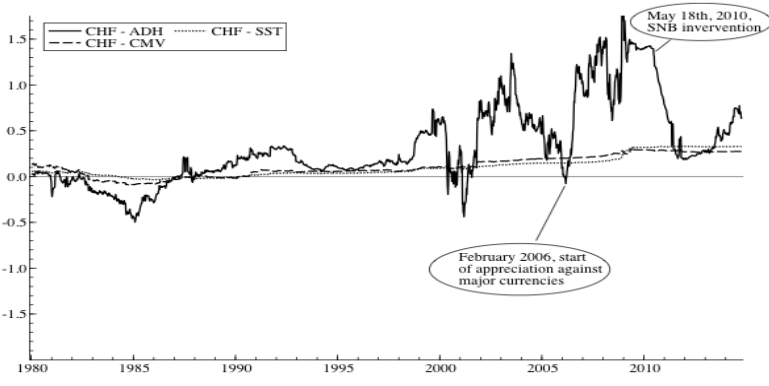

GBP Exposures

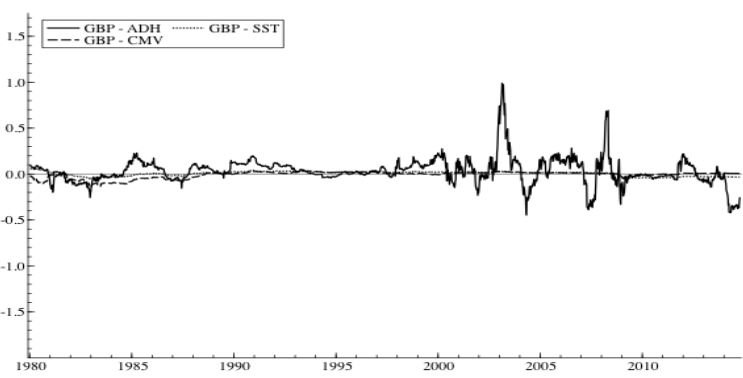

SEK Exposures

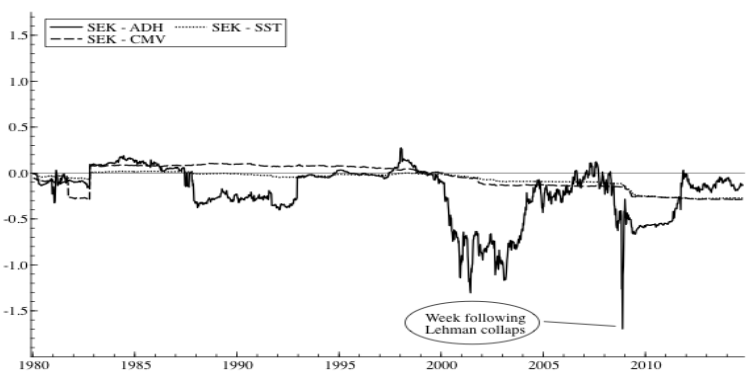

Figure 1: Exposure plots. This figure depicts the optimal currency exposure to the CAD, CHF, EUR, GBP, JPY, and SEK, respectively, computed using three different hedging methods and a weekly rebalancing horizon. CMV (dashed) is a real-time implementation of the procedure in Campbell et al. (2010), SST (dotted) is the semistatic implementation using quadratic covariation estimates, whereas ADH (line) is a dynamic implementation of the realized currency beta hedging strategy, described in Section 4.2. Note that the $x$-axis is slightly inaccurate as it is based on a count to 52 weeks per year and does not account for extra days due to leap years. Hence, there are seven additional weeks relative to the count. This out-of-sample period covers January 1980 through August 2014. 


\section{Full and RCB}

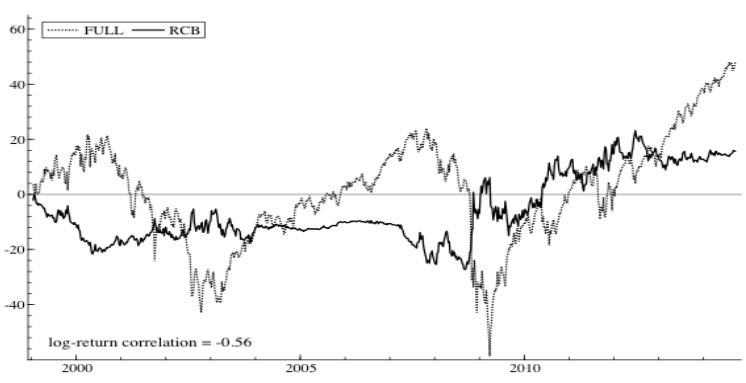

Momentum and RCB

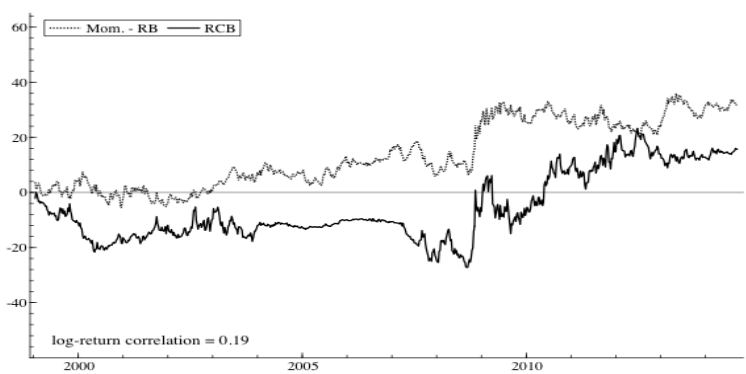

Carry and RCB

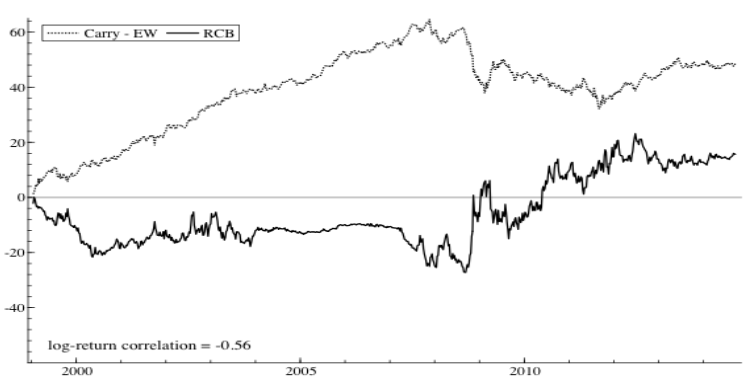

Value and RCB

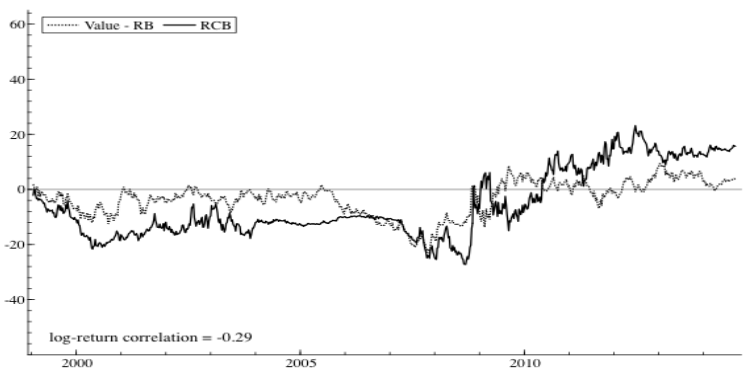

Figure 2: Cumulative log-returns. This figure depicts the cumulative log-return to the currency hedging part of the ADH strategy applied to the fully hedged S\&P 500 portfolio, labelled RCB (line), together with the cumulative log-returns on the S\&P 500, a carry trade strategy using equal weights, a currency momentum strategy using rankbased weights, and a currency value strategy using rank-based weights (all dotted). The implementation of such strategies are detailed in the supplementary appendix. Moreover, the correlations between the log-returns of RCB and the four alternatives are also provided. Due to initialization of the value strategy and the ADH procedure, the overlapping sample is confined to January 1999 through August 2014. Note that the $x$-axis is slightly inaccurate as it is based on a count to 52 weeks per year and does not account for extra days due to leap years. Hence, there are four additional weeks relative to the count. Finally, the scale on the $y$-axis is (the sum of) weekly percentage points. 
CAD: MSCI and S\&P 500

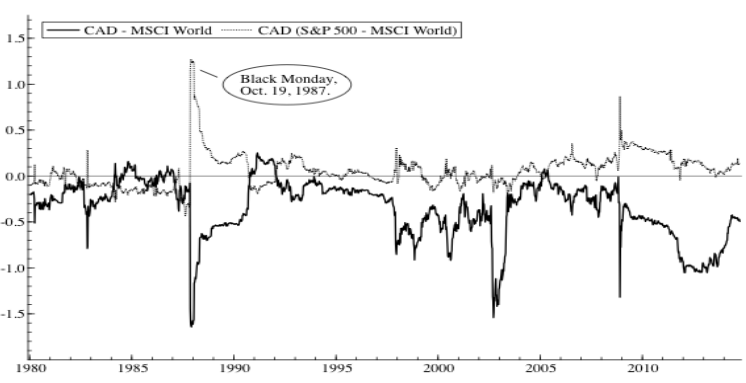

EUR: MSCI and S\&P 500

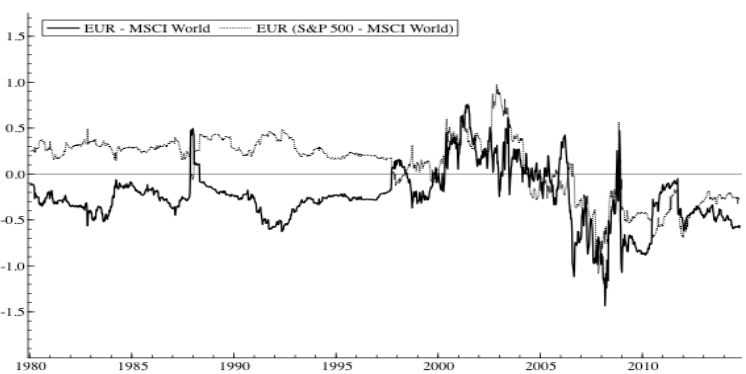

JPY: MSCI and S\&P 500

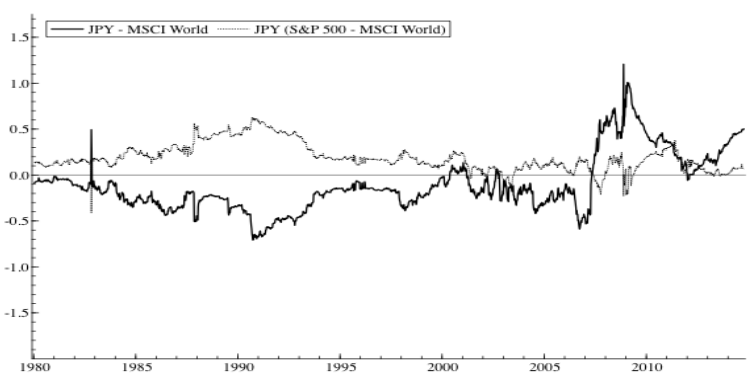

CHF: MSCI and S\&P 500

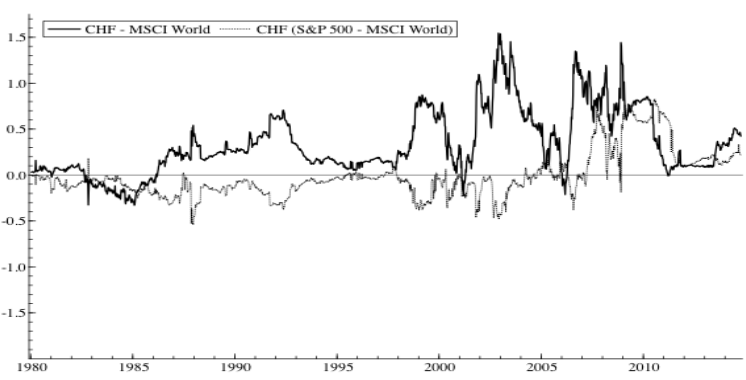

GBP: MSCI and S\&P 500

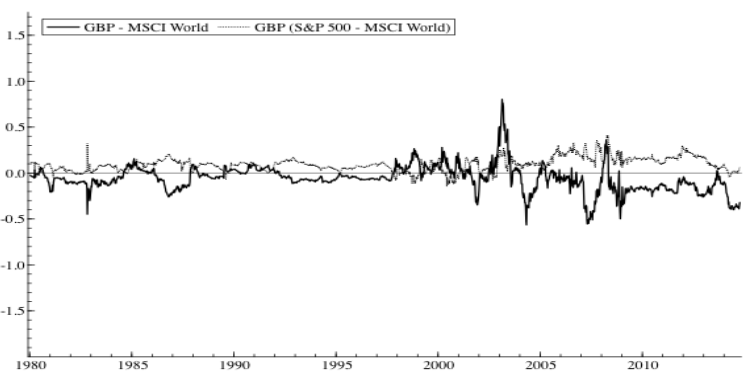

SEK: MSCI and S\&P 500

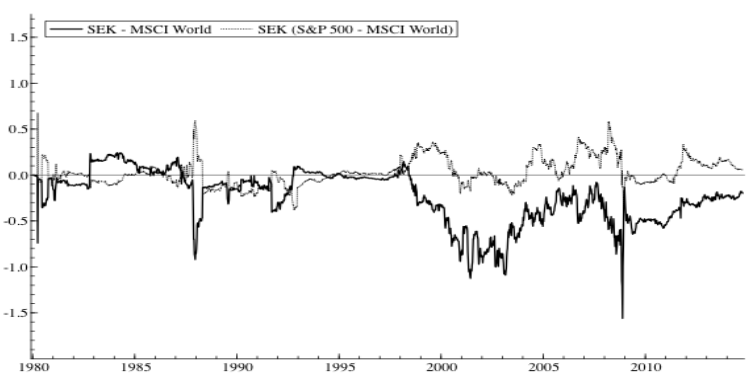

Figure 3: Global equity realized currency betas. This figure depicts the optimal currency exposure to the CAD, CHF, EUR, GBP, JPY, and SEK, computed using the ADH strategy, a dynamic implementation of the realized currency beta hedging strategy described in Section 4.2, applied to the MSCI World equity portfolio (line). The difference between these estimates and the corresponding optimal exposures for the S\&P 500 portfolio is also provided (dotted). Note that the $x$-axis is slightly inaccurate as it is based on a count to 52 weeks per year and does not account for extra days due to leap years. Hence, there are seven additional weeks relative to the count. This out-of-sample period covers January 1980 through August 2014. 

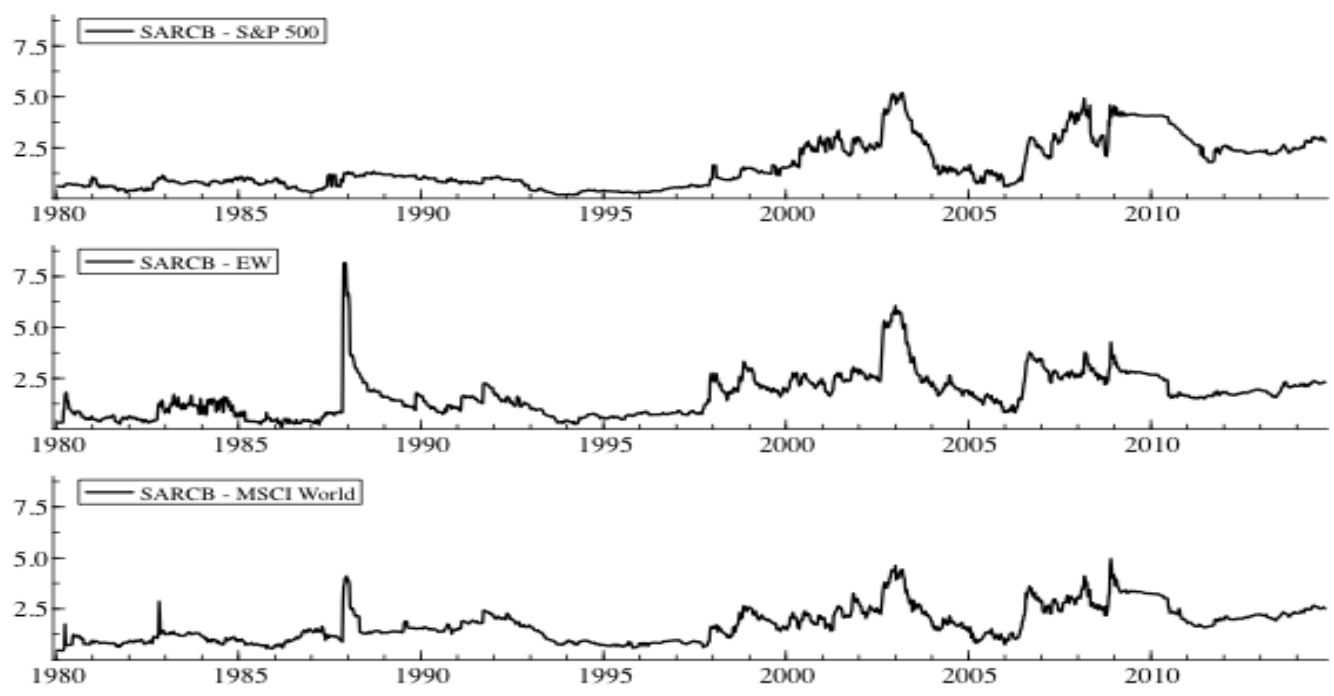

Figure 4: Sum of absolute realized currency betas. This figure illustrates the sum of the absolute optimal currency exposures to the CAD, CHF, EUR, GBP, JPY, and SEK, computed using the ADH strategy, a dynamic implementation of the realized currency beta hedging strategy described in Section 4.2, applied to either the S\&P 500 , EW, or MSCI World equity portfolio. Note that the $x$-axis is slightly inaccurate as it is based on a count to 52 weeks per year and does not account for extra days due to leap years. Hence, there are seven additional weeks relative to the count. This out-of-sample period covers January 1980 through August 2014. 
CAD: HF vs. Daily

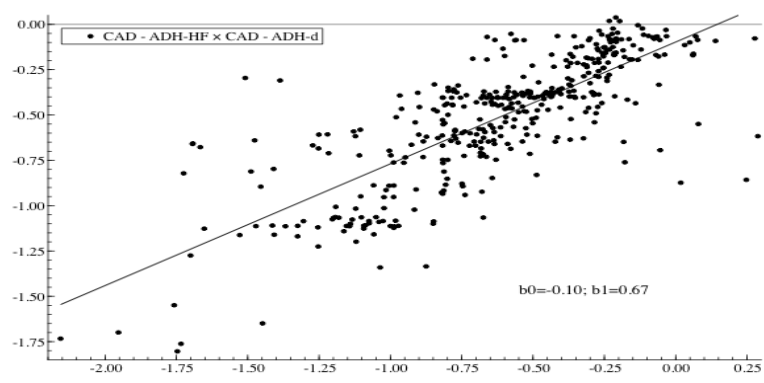

EUR: HF vs. Daily

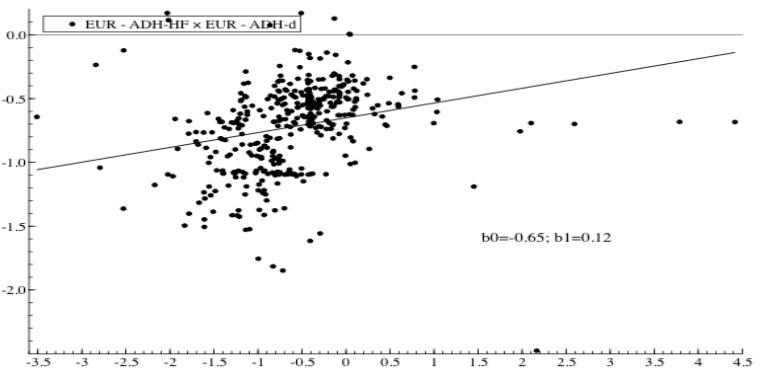

JPY: HF vs. Daily

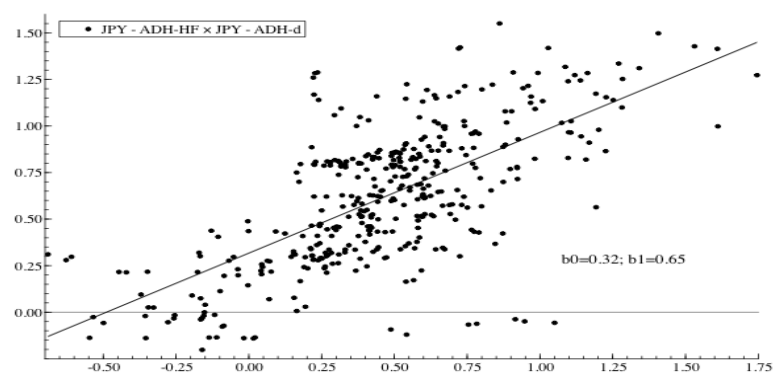

CHF: HF vs. Daily

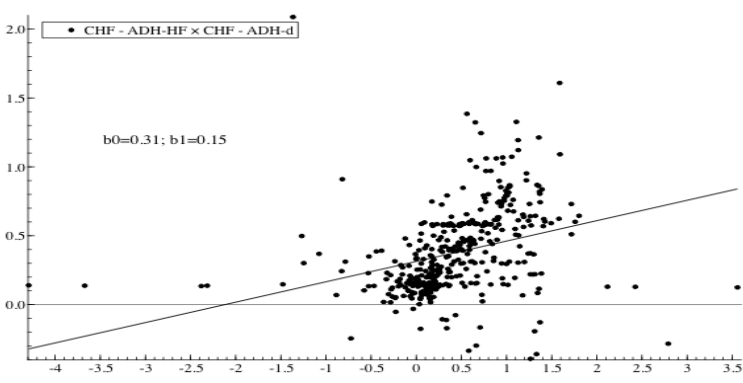

GBP: HF vs. Daily

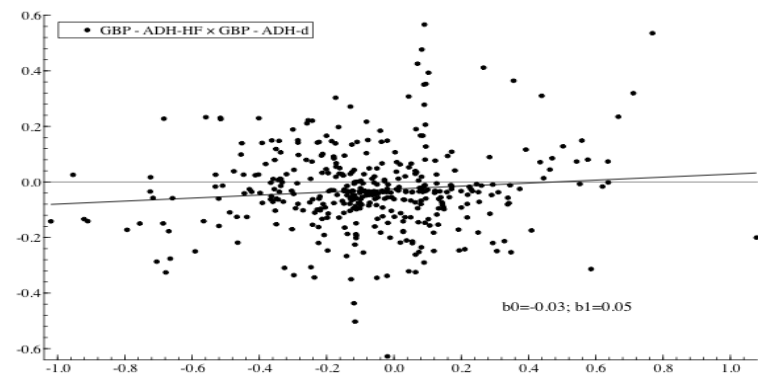

USD: HF vs. Daily

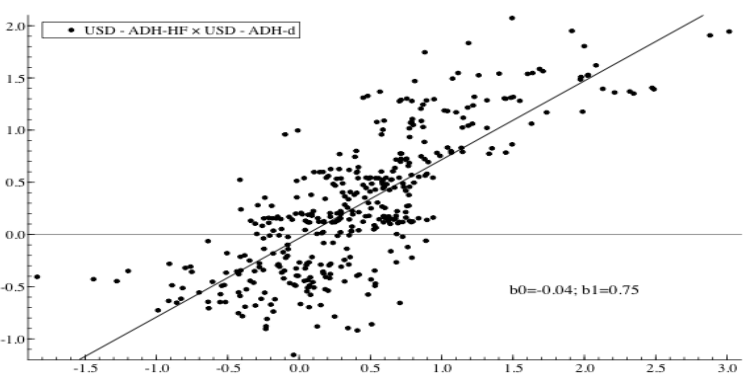

Figure 5: High-frequency versus daily exposures. This figure provides scatter plots of the optimal currency exposures to the CAD, CHF, EUR, GBP, and JPY, along with the implicit exposure to the USD. These are computed using either intra-daily ( $y$-axis) or daily ( $x$-axis) data based quadratic covariation estimates in combination with the $\mathrm{ADH}$ strategy for a weekly rebalancing horizon. A linear regression fit is included in all plots, along with estimates of the constant $\left(b_{0}\right)$ and slope $\left(b_{1}\right)$. This out-of-sample period covers September 2006 though August 2014. 

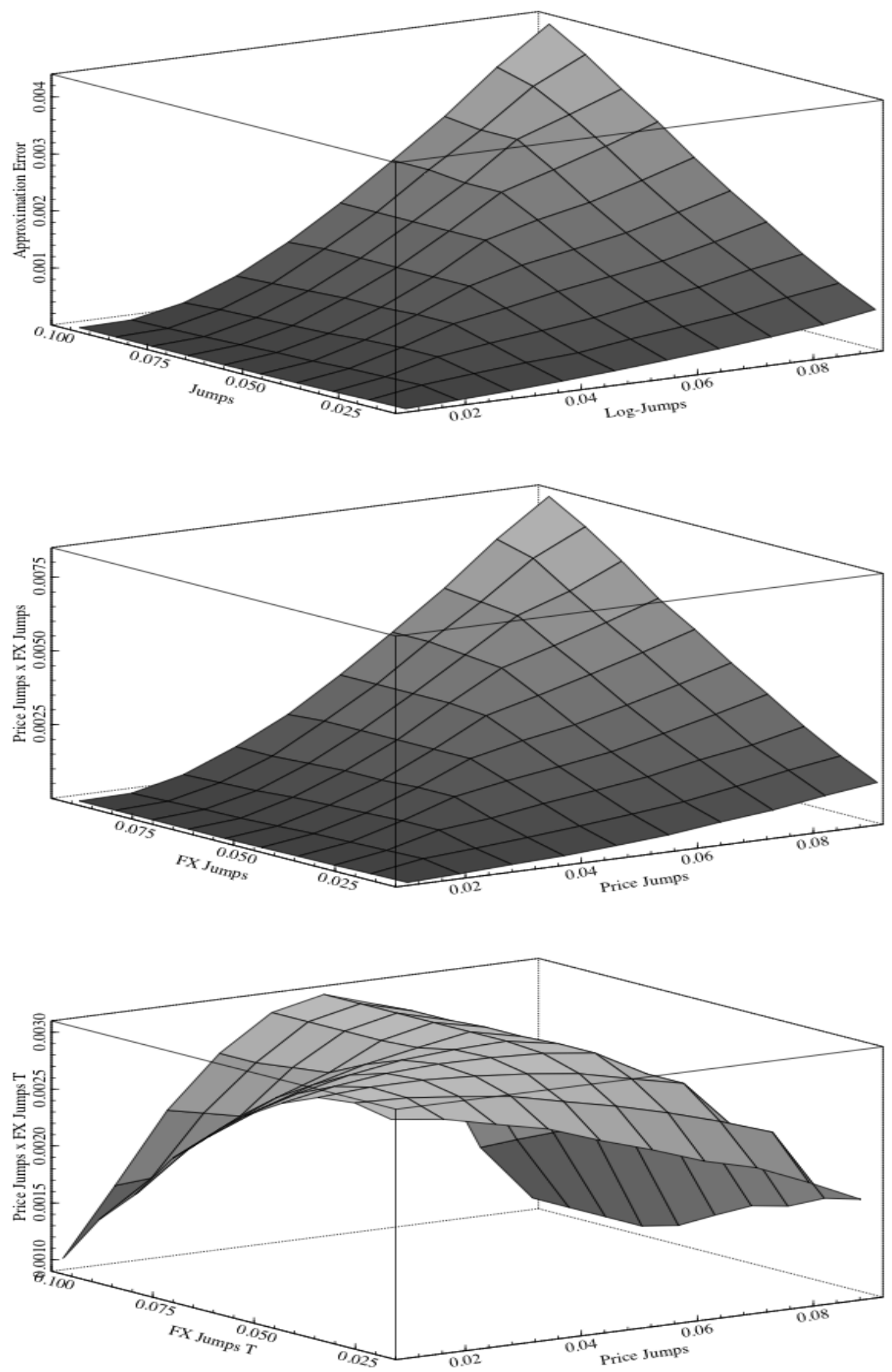

Figure 6: Approximation errors. This figure illustrates the logarithmic jump approximation errors from applying Itô's lemma and co-jump errors for various jump sizes. In particular, Panel A shows the logarithmic jump approximation errors for log-jumps $\left(d_{c \tau}, k_{c, \tau}\right)$ of size [0,0.1]. Panel B, on the other hand, provides the co-jump errors where both $d_{c \tau}$ and $k_{c, \tau}$ are linearly increasing in the interval [0,0.1], and Panel C provides similar errors where $k_{c, \tau}$ are now linearly decreasing in $[0,0.1]$. The errors entering $\tilde{J}_{\tau}^{e}$ is provided on the $z$-axis in all cases. 


\section{References}

Aït-Sahalia, Y. \& Jacod, J. (2012), 'Analyzing the spectrum of asset returns: Jump and volatility components in high frequency data', Journal of Economic Literature 50, 1007-1050.

Akram, F., Rime, D. \& Sarno, L. (2008), 'Arbitrage in the foreign exchange market: Turning on the microscope', Journal of International Economics 76, 237-253.

Andersen, T. G. \& Benzoni, L. (2012), Stochastic volatility, in R. A. Meyers, ed., 'Encyclopedia of Complexity and Systems Science', Springer-Verlag. forthcoming.

Andersen, T. G. \& Bollerslev, T. (1998), 'Answering the skeptics: Yes, standard volatility models do provide accurate forecasts', International Economic Review 39, 885-905.

Andersen, T. G., Bollerslev, T., Christoffersen, P. F. \& Diebold, F. X. (2013), Financial risk measurement for financial risk management, in G. M. Constantinides, M. Harris \& R. M. Stulz, eds, 'Handbook of the Economics of Finance', North Holland, pp. 1127-1220.

Andersen, T. G., Bollerslev, T. \& Diebold, F. X. (2010), Parametric and nonparametric measurements of volatility, in Y. Ait-Sahalia \& L. P. Hansen, eds, 'Handbook of Financial Econometrics', Elsevier, North Holland.

Andersen, T. G., Bollerslev, T., Diebold, F. X. \& Ebens, H. (2001), 'The distribution of realized stock return volatility', Journal of Financial Economics 61, 43-76.

Andersen, T. G., Bollerslev, T., Diebold, F. X. \& Labys, P. (2001), 'The distribution of exchange rate volatility', Journal of the American Statistical Association 96, 42-55.

Andersen, T. G., Bollerslev, T., Diebold, F. X. \& Labys, P. (2003), 'Modeling and forecasting realized volatility', Econometrica 71, 579-625.

Andersen, T. G., Dobrev, D. \& Schaumburg, E. (2012), 'Jump-robust volatility estimation using nearest neighbor truncation', Journal of Econometrics 169, 75-93.

Anderson, R. W. \& Danthine, J.-P. (1981), 'Cross hedging', Journal of Political Economy 89, 11821196.

Andreou, E. \& Ghysels, E. (2002), 'Rollling-sample volatility estimators: Some new theoretical, simulation, and empirical results', Journal of Business and Economic Statistics 20, 363-377.

Asness, C. S., Moskowitz, T. J. \& Pedersen, L. H. (2013), 'Value and momentum everywhere', The Journal of Finance 68, 929-985.

Back, K. (1991), 'Asset pricing for general processes', Journal of Mathematical Economics 20, 371-395. 
Baillie, R. \& Bollerslev, T. (1989), 'Common stochastic trends in a system of exchange rates', The Journal of Finance 44, 167-181.

Bandi, F. M. \& Russell, J. R. (2008), 'Microstructure noise, realized variance, and optimal sampling', Review of Economic Studies 75, 339-369.

Barndorff-Nielsen, O. E. \& Shephard, N. (2004a), 'Econometric analysis of realised covariation: High frequency based covariance, regression and correlation in financial economics', Econometrica 72, 885-925.

Barndorff-Nielsen, O. E. \& Shephard, N. (2004b), 'Power and Bipower Variation with Stochastic Volatility and Jumps', Journal of Financial Econometrics 2, 1-37.

Barndorff-Nielsen, O. E. \& Shephard, N. (2007), Variation, jumps, market frictions and high frequency data in financial econometrics, in R. Blundell, T. Persson \& W. K. Newey, eds, 'Advances in Economics and Econometrics: Theory and Applications, Ninth World Congress', Cambridge University Press, Cambridge, UK, pp. 328-372.

Bekaert, G., Hodrick, R. \& Zhang, X. (2009), 'International stock return comovements', The Journal of Finance 64, 2591-2626.

Black, F. (1976), Studies of stock price volatility changes, in 'Proceedings of the 1976 Business Meeting of the Business and Economic Statistics Section, American Statistical Association', pp. 177-181.

Bollerslev, T. \& Wright, J. H. (2000), 'Semiparametric estimation of long-memory volatility dependencies: The role of high-frequency data', Journal of Econometrics 98, 81-106.

Bos, C. S., Mahieu, R. J. \& van Dijk, H. K. (2000), 'Daily exchange rate behavior and hedging of currency risk', Journal of Applied Econometrics 15, 671-696.

Britten-Jones, M. (1999), 'The sampling error in estimates of mean-variance efficient portfolio weights', The Journal of Finance 54, 655-671.

Brunnermeier, M. K., Nagel, S. \& Pedersen, L. H. (2009), 'Carry trades and currency crashes', NBER Macroeconomics Annual 23, 313-348.

Burnside, C., Eichenbaum, M., Kleshchelski, I. \& Rebelo, S. (2011), 'Do peso problems explain the returns to the carry trade?', Review of Financial Studies 24, 853-891.

Campbell, J. Y., de Medeiros, K. S. \& Viceira, L. M. (2010), 'Global currency hedging', The Journal of Finance 65, 87-121.

Chiriac, R. \& Voev, V. (2011), 'Modelling and forecasting multivariate realized volatility', Journal of Applied Econometrics 28, 922-947. 
Christensen, B. J. \& Varneskov, R. T. (2015), 'Dynamic global currency hedging: Supplementary appendix'. Unpublished manuscript, Northwestern University.

Christie, A. A. (1982), 'The stochastic behavior of common stock variances - value, leverage and interest rate effects', Journal of Financial Economics 10, 407-432.

Christoffersen, P., Errunza, V. R., Jacobs, K. \& Langlois, H. (2012), 'Is the potential for international diversification disappearing? a dynamic copula approach', Review of Financial Studies 25, 37113751 .

Comte, F. \& Renault, E. (1998), 'Long memory in continuous-time stochastic volatility models', Mathematical Finance 8, 291-323.

Cont, R. \& Tankov, P. (2004), Financial Modelling With Jump Processes, Chapman \& Hall/CRC Financial Mathematics Series, Boca Raton.

de Roon, F. A., Nijman, T. E. \& Werker, B. J. (2003), 'Currency hedging for international portfolios: The usefulness of mean-variance analysis', Journal of Banking and Finance 27, 327-349.

Delbaen, F. \& Schachermayer, W. (1994), 'A general version of the fundamental theorem of asset pricing', Mathematische Annalen 300, 463-520.

Della Corte, P., Sarno, L. \& Tsiakas, I. (2009), 'An economic evaluation of empirical exchange rate models', Review of Financial Studies 22, 3491-3530.

Engle, R. F. \& Ng, V. K. (1993), 'Measuring and testing the impact of news in volatility', Journal of Finance 43, 1749-1778.

Errunza, V., Hogan, K. \& Hung, M.-W. (1999), 'Can the gains to international diversification be achieved without trading abroad?', The Journal of Finance 54, 2075-2107.

Eun, C. S., Huang, W. \& Lai, S. (2008), 'International diversification with large- and small-cap stocks', Journal of Financial and Quantitative Analysis 43, 489-523.

Eun, C. S., Lai, S., de Roon, F. A. \& Zhang, Z. (2010), 'International diversification with factor funds', Management Science 56, 1500-1518.

Eun, C. S. \& Lee, J. (2010), 'Mean-variance convergence around the world', Journal of Banking and Finance 34, 856-870.

Fama, E. F. \& French, K. R. (2012), 'Size, value, and momentum in international stock returns', Journal of Financial Economics 105, 457-472.

Fleming, J., Kirby, C. \& Ostdiek, B. (2001), 'The economic value of volatility timing', Journal of Finance 56, 329-352. 
Fleming, J., Kirby, C. \& Ostdiek, B. (2003), "The economic value of volatility timing using "realized" volatility', Journal of Financial Economics 67, 473-509.

Foster, D. P. \& Nelson, D. B. (1996), 'Continuous record asymptotics for rolling sample variance estimators', Econometrica 64, 139-174.

Glen, J. \& Jorion, P. (1993), 'Currency hedging for international portfolios', The Journal of Finance 48, 1865-1886.

Gloter, A. \& Jacod, J. (2001a), 'Diffusions with measurement errors. 1 - local asymptotic normality.', ESAIM: Probability and Statistics 5, 225-242.

Gloter, A. \& Jacod, J. (2001b), 'Diffusions with measurement errors. 2 - measurement errors.', ESAIM: Probability and Statistics 5, 243-260.

Grubel, H. G. (1968), 'Internationally diversified portfolios: Welfare gains and capital flows', The American Economic Review 58, 1299-1314.

Hampel, F. R. (1974), 'The influence curve and its role in robust estimation', Journal of the American Statistical Association 69, 383-293.

Han, Y. (2006), 'Asset allocation with a high dimensional latent factor stochastic volatility model', Review of Financial Studies 19, 237-271.

Hansen, P. R. \& Lunde, A. (2006), 'Realized variance and market microstructure noise', Journal of Business and Economic Statistics 24, 127-161.

Holm, S. (1979), 'A simple sequentially rejective multiple test procedure', Scandinavian Journal of Statistics 6, 65-70.

Huang, X. \& Tauchen, G. (2005), 'The relative contribution of jumps to total price variance', Journal of Financial Econometrics 3, 456-499.

Jacod, J. (2012), Statistics and high frequency data, in M. Kessler, A. Lindner \& M. Sørensen, eds, 'Statistical Methods for Stochastic Differential Equations', CRC Press.

Jacod, J. \& Shiryaev, A. N. (2003), Limit Theorems for Stochastic Processes, 2nd Edition, SpringerVerlag: New-York.

Jagannathan, R. \& Ma, T. (2003), 'Risk reduction in large portfolios: Why imposing the wrong constraints helps', The Journal of Finance 58, 1651-1684.

Kan, R. \& Zhou, G. (2012), 'Tests of mean-variance spanning', Annals of Economics and Finance 13, 145-193. 
Karolyi, G. A. \& Stulz, R. M. (2003), Are financial assets priced locally or globally?, in G. M. Constantinides, M. Harris \& R. M. Stulz, eds, 'Handbook of the Economics of Finance', Elsevier, pp. $975-1020$.

Kroencke, T. A., Schindler, F. \& Schrimpf, A. (2014), 'International diversification benefits with foreign exchange rate styles', Review of Finance 18, 18471883.

Kroner, K. F. \& Sultan, J. (1993), 'Time-varying distributions and dynamic hedging with foreign currency futures', Journal of Financial and Quantitative Analysis 28, 535-551.

Ledoit, O. \& Wolf, M. (2008), 'Robust performance hypothesis testing with the sharpe ratio', Journal of Empirical Finance 15, 850-859.

Ledoit, O. \& Wolf, M. (2011), 'Robust performance hypothesis testing with the variance', Wilmott Magazine 55, 86-89.

Levy, H. \& Sarnat, M. (1970), 'International diversification of investment portfolios', The American Economic Review 60, 668-675.

Liu, Q. (2009), 'On portfolio optimization: How and when do we benefit from high-frequency data?', Journal of Applied Econometrics 24, 560-582.

Longin, F. \& Solnik, B. (1995), 'Is the correlation in international equity returns constant: 1960-1990?', Journal of International Money and Finance 14, 3-26.

Lustig, H., Roussanov, N. \& Verdelhan, A. (2011), 'Common risk factors in currency markets', Review of Financial Studies 24, 3731-3777.

Lustig, H., Roussanov, N. \& Verdelhan, A. (2014), 'Countercyclical currency risk premia', Journal of Financial Economics 111, 527-553.

Lustig, H. \& Verdelhan, A. (2007), 'The cross section of foreign currency risk premia and consumption growth risk', The American Economic Review 97, 89-117.

Mancini, L., Ranaldo, A. \& Wrampelmeyer, J. (2013), 'Liquidity in the foreign exchange market: Measurement, commonality, and risk premiums', The Journal of Finance 68, 1805-1841.

Marquering, W. \& Verbeek, M. (2004), 'The economic value of predicting stock index returns and volatility', Journal of Financial and Quantitative Analysis 39, 407-429.

Martens, M. (2004), Estimating unbiased and precise realized covariances. Econometric Institute, Erasmus University Rotterdam.

Meddahi, N. (2002), 'A theoretical comparison between integrated and realized volatility', Journal of Applied Econometrics 17, 479-508. 
Menkhoff, L., Sarno, L., Schmeling, M. \& Schrimpf, A. (2012a), 'Carry trades and global foreign exchange volatility', The Journal of Finance 67, 681-718.

Menkhoff, L., Sarno, L., Schmeling, M. \& Schrimpf, A. (2012b), 'Currency momentum strategies', Journal of Financial Economics 106, 660-684.

Moskowitz, T. J., Ooi, Y. H. \& Pedersen, L. H. (2012), 'Time series momentum', Journal of Financial Economics 104, 228-250.

Mykland, P. A. (2010), 'A Gaussian calculus for incerence from high frequency data', Annals of Finance 8, 235-258.

Øksendal, B. \& Sulem, A. (2007), Applied Stochastic Control of Jump Diffusions, 2nd Edition, Springer Verlag: New York.

Opie, W. \& Dark, J. (2015), 'Currency overlay for global equity portfolios: Cross-hedging and base currency', Journal of Futures Markets 35, 186-200.

Ramadorai, T. (2008), 'What determines transaction costs in foreign exchange markets?', International Journal of Finance and Economics 13, 14-25.

Ranaldo, A. \& Söderlind, P. (2010), 'Safe haven currencies', Review of Finance 14, 385-407.

Romano, J. P., Shaikh, A. Z. \& Wolf, M. (2010), 'Hypothesis testing in econometrics', Annual Review of Economics 2, 75-104.

Schmittmann, J. M. (2010), Currency hedging for international portfolios. IMF working paper, WP$10-151$.

Solnik, B. H. (1974), 'Why not diversify internationally rather than domestically?', Financial Analysts Journal 30, 48-54.

Varneskov, R. T. (2015a), Estimating the quadratic variation spectrum of noisy asset prices using generalized flat-top realized kernels. Unpublished Manuscript, Aarhus University.

Varneskov, R. T. (2015b), 'Flat-top realized kernel estimation of quadratic covariation with nonsynchronous and noisy asset prices', Journal of Business and Economic Statistics forthcoming.

Varneskov, R. T. \& Voev, V. (2013), 'The role of realized ex-post covariance measures and dynamic model choice on the quality of covariance forecasts', Journal of Empirical Finance 20, 83-95.

Voev, V. \& Lunde, A. (2007), 'Integrated covariance estimation using high-frequency data in the presence of noise', Journal of Financial Econometrics 5, 68-104.

Yu, J. (2005), 'On leverage in a stochastic volatility model', Journal of Econometrics 127, 165-178. 


\section{A Proofs of Asymptotic Results}

This section contains the proofs of Propositions 1-4 in the main text.

\section{A.1 Proof of Proposition 1}

Before describing the dynamics of $d v_{\tau}$, it is pertinent to characterize the diffusive behavior of the different components of $d V_{\tau} / V_{\tau}$ in (6). First, the system of equity, bond, and currency prices in equations (3)-(5) may be rewritten using Itô's Lemma as

$$
\begin{aligned}
& d p_{c, \tau}=d P_{c, \tau} / P_{c, \tau}-\left(\sigma_{c, \tau}^{2} / 2\right) d \tau+o_{p}(d \tau) \\
& d s_{c, \tau}=d S_{c, \tau} / S_{c, \tau}-\left(\varphi_{c, \tau}^{2} / 2\right) d \tau+o_{p}(d \tau) \\
& d b_{c, \tau}=d B_{c, \tau} / B_{c, \tau}=\lambda_{c, \tau} d \tau
\end{aligned}
$$

for all $c=0, \ldots, C$ countries. Next, by Itô's product rule, write

$$
\begin{aligned}
\frac{d P_{c, \tau} S_{c, \tau}}{P_{c, \tau} S_{c, \tau}} & =\frac{d P_{c, \tau}}{P_{c, \tau}}+\frac{d S_{c, \tau}}{S_{c, \tau}}+\left[d P_{c, \tau} / P_{c, \tau}, d S_{c, \tau} / S_{c, \tau}\right]+o_{p}(d \tau) \\
& =d p_{c, \tau}+d s_{c, \tau}+\left[d p_{c, \tau}+d s_{c, \tau}\right] / 2+o_{p}(d \tau),
\end{aligned}
$$

using (A.1)-(A.2), and, similarly, by Itô's quotient rule, write

$$
\frac{d B_{0, \tau} B_{c, \tau}}{B_{0, \tau} B_{c, \tau}}=d b_{0, \tau}-d b_{c, \tau}=\left(\lambda_{0, \tau}-\lambda_{c, \tau}\right) d \tau
$$

using (A.3). Then, inserting (A.2), (A.4), and (A.5) into the expression for $d V_{\tau} / V_{\tau}$ in (6),

$$
\begin{aligned}
\frac{d V_{\tau}}{V_{\tau}}= & \sum_{c=0}^{C} w_{c, t}\left(d p_{c, \tau}+d s_{c, \tau}+\left[d p_{c, \tau}+d s_{c, \tau}\right] / 2\right)+\sum_{c=0}^{C} \theta_{c, t}\left(\lambda_{0, \tau}-\lambda_{c, \tau}\right) d \tau \\
& -\sum_{c=0}^{C} \theta_{c, t}\left(d s_{c, \tau}+\left(\varphi_{c, \tau}^{2} / 2\right) d \tau\right)+o_{p}(d \tau) \\
= & \boldsymbol{w}_{t}^{\prime}\left(d \boldsymbol{p}_{\tau}+d \boldsymbol{s}_{\tau}\right)-\boldsymbol{\Theta}_{t}^{\prime}\left(d \boldsymbol{s}_{\tau}-\boldsymbol{\lambda}_{0, \tau} d \tau+\boldsymbol{\lambda}_{\tau} d \tau\right)+\sum_{\tau}^{h, 1} d \tau+o_{p}(d \tau)
\end{aligned}
$$

where the second equality follows by re-writing $d V_{\tau} / V_{\tau}$ on matrix form and defining $\Sigma_{\tau}^{h, 1}$ as

$$
\begin{aligned}
\sum_{\tau}^{h, 1} & =\sum_{c=0}^{C} w_{c, t}\left(\sigma_{c, \tau}^{2}+\varphi_{c, \tau}^{2}+2 \psi_{c, c, \tau}\right) / 2-\sum_{c=0}^{C} \theta_{c, t} \varphi_{c, \tau}^{2} / 2 \\
& =\left(\boldsymbol{w}_{t}^{\prime} \operatorname{diag}\left(\left[d \boldsymbol{p}_{\tau}+d \boldsymbol{s}_{\tau}\right]\right)-\boldsymbol{\Theta}_{t}^{\prime} \operatorname{diag}\left(\left[d \boldsymbol{s}_{\tau}\right]\right)\right) /(2 d \tau)
\end{aligned}
$$

with $\operatorname{diag}(\boldsymbol{A})$ denoting the row vector containing the diagonal of a given symmetric matrix $\boldsymbol{A}$. Here, it follows immediately that $\Sigma_{\tau}^{h, 1}$ is locally bounded, cádlág, and $\mathcal{F}_{\tau}$-adapted by Assumptions 1-2. Another 
application of Itô's Lemma gives $d v_{\tau}=d V_{\tau} / V_{\tau}-\left[d V_{\tau} / V_{\tau}\right] / 2+o_{p}(d \tau)$, which, since $\left[\Sigma_{\tau}^{h, 1} d \tau\right]=o_{p}(d \tau)$ by the properties of $\Sigma_{\tau}^{h, 1}$, implies

$$
d v_{\tau}=\boldsymbol{w}_{t}^{\prime}\left(d \boldsymbol{p}_{\tau}+d \boldsymbol{s}_{\tau}\right)-\boldsymbol{\Theta}_{t}^{\prime}\left(d \boldsymbol{s}_{\tau}-\boldsymbol{\lambda}_{0, \tau} d \tau+\boldsymbol{\lambda}_{\tau} d \tau\right)+\Sigma_{\tau}^{h, 1} d \tau+\Sigma_{\tau}^{h, 2} d \tau+o_{p}(d \tau)
$$

where $\Sigma_{\tau}^{h, 2} \equiv-\left[d V_{\tau} / V_{\tau}\right] /(2 d \tau)$ with

$$
\begin{aligned}
-\left[d V_{\tau} / V_{\tau}\right] /(2 d \tau) & =-\left[\boldsymbol{w}_{t}^{\prime}\left(d \boldsymbol{p}_{\tau}+d \boldsymbol{s}_{\tau}\right)-\boldsymbol{\Theta}_{t}^{\prime} d \boldsymbol{s}_{\tau}\right] /(2 d \tau)+o_{p}(1) \\
& =-\boldsymbol{w}_{t}^{\prime}\left(\boldsymbol{\sigma}_{\tau}+\boldsymbol{\varphi}_{\tau}+2 \boldsymbol{\Psi}_{\tau}\right) \boldsymbol{w}_{t} / 2-\boldsymbol{\Theta}_{t}^{\prime} \boldsymbol{\varphi}_{\tau} \boldsymbol{\Theta}_{t} / 2+\boldsymbol{w}_{t}^{\prime}\left(\boldsymbol{\varphi}_{\tau}+\boldsymbol{\Psi}_{\tau}\right) \boldsymbol{\Theta}_{t}+o_{p}(1)
\end{aligned}
$$

using the definitions $\boldsymbol{\Sigma}_{\tau}=\left(\sigma_{c, k, \tau}\right)_{0 \leq c, k \leq C} \mathbf{1}_{\{c \neq k\}}+\left(\sigma_{c, \tau}^{2}\right)_{c=0, \ldots, C} \mathbf{1}_{\{c=k\}}$, with $\mathbf{1}_{\{\cdot\}}$ being the indicator function, $\boldsymbol{\varphi}_{\tau}=\left(\varphi_{c, k, \tau}\right)_{0 \leq c, k \leq C} \mathbf{1}_{\{c \neq k\}}+\left(\varphi_{c, \tau}^{2}\right)_{c=0, \ldots, C} \mathbf{1}_{\{c=k\}}$, and $\boldsymbol{\Psi}_{\tau}=\left(\Psi_{c, k, \tau}\right)_{0 \leq c, k \leq C}$ for the instantaneous quadratic covariation matrices of the equity prices $\boldsymbol{p}_{\tau}$, foreign exchange rates $\boldsymbol{s}_{\tau}$, and between $\boldsymbol{p}_{\tau}$ and $\boldsymbol{s}_{\tau}$, respectively. Lastly, define $\Sigma_{\tau}^{h}=\Sigma_{\tau}^{h, 1}+\Sigma_{\tau}^{h, 2}$, then this is locally bounded, cádlág, and $\mathcal{F}_{\tau}$-adapted by Assumptions 1-2, since $\Sigma_{\tau}^{h, 2}$ satisifes these properties well. The final representation is found by a standard addition and subtraction argument, using the definition $\boldsymbol{\beta}_{t}=\boldsymbol{w}_{t}-\boldsymbol{\Theta}_{t}$.

\section{A.2 Proof of Proposition 2}

This result follows immediately since $\left\|\boldsymbol{w}_{t}\right\|+\left\|\boldsymbol{\beta}_{t}\right\|<\infty$ by Assumption 2, $\|\cdot\|$ being the Euclidean matrix norm, $\boldsymbol{\lambda}_{\tau}$ is locally bounded and $\mathcal{F}_{\tau}$-predictable by Assumption 1, and, finally, since the Jensen's correction, $\Sigma_{\tau}^{h}$, is $\mathcal{F}_{\tau}$-adapted, locally bounded, and cádlág by Proposition 1.

\section{A.3 Proof of Proposition 3}

First, since $\tilde{\boldsymbol{\beta}}_{t}$ spans the unique elements of $\boldsymbol{\beta}$, the objective function in the minimization problem may be simplified as

$$
\underset{\beta_{t} \mid \boldsymbol{w}_{t}}{\operatorname{argmin}} \mathcal{L}_{t}\left(\boldsymbol{\beta}_{t}, \boldsymbol{w}_{t}\right)=\underset{\tilde{\boldsymbol{\beta}}_{t} \mid \boldsymbol{w}_{t}}{\operatorname{argmin}} \tilde{\mathcal{L}}_{t}\left(\tilde{\boldsymbol{\beta}}_{t}, \boldsymbol{w}_{t}\right)
$$

with

$$
\tilde{\mathcal{L}}_{t}\left(\tilde{\boldsymbol{\beta}}_{t}, \boldsymbol{w}_{t}\right)=\mathbb{E}_{t}\left[\left[\boldsymbol{w}_{t}^{\prime} d \boldsymbol{p}_{\tau}\right]_{t+1}\right] / 2+\tilde{\boldsymbol{\beta}}_{t}^{\prime} \mathbb{E}_{t}\left[\left[d \tilde{\boldsymbol{s}}_{\tau}\right]_{t+1}\right] \tilde{\boldsymbol{\beta}}_{t} / 2+\mathbb{E}_{t}\left[\left[\boldsymbol{w}_{t}^{\prime} d \boldsymbol{p}_{\tau}, d \tilde{\boldsymbol{s}}_{\tau}\right]_{t+1}\right] \tilde{\boldsymbol{\beta}}_{t} .
$$

Hence, by standard matrix calculus,

$$
\frac{\partial \tilde{\mathcal{L}}_{t}\left(\tilde{\boldsymbol{\beta}}_{t}, \boldsymbol{w}_{t}\right)}{\partial \tilde{\boldsymbol{\beta}}_{t}}=\tilde{\boldsymbol{\beta}}_{t}^{\prime} \mathbb{E}_{t}\left[\left[d \tilde{\boldsymbol{s}}_{\tau}\right]_{t+1}\right]+\mathbb{E}_{t}\left[\left[\boldsymbol{w}_{t}^{\prime} d \boldsymbol{p}_{\tau}, d \tilde{\boldsymbol{s}}_{\tau}\right]_{t+1}\right], \quad \frac{\partial^{2} \tilde{\mathcal{L}}_{t}\left(\tilde{\boldsymbol{\beta}}_{t}, \boldsymbol{w}_{t}\right)}{\partial \tilde{\boldsymbol{\beta}}_{t} \partial \tilde{\boldsymbol{\beta}}_{t}^{\prime}}=\mathbb{E}_{t}\left[\left[d \tilde{\boldsymbol{s}}_{\tau}\right]_{t+1}\right],
$$

which readily yields the desired minimum solution for $\tilde{\boldsymbol{\beta}}_{t}$, since $\mathbb{E}_{t}\left[\left[d \tilde{\boldsymbol{s}}_{\tau}\right]_{t+1}\right]$ is positive definite. 


\section{A.4 Proof of Proposition 4}

Similarly to the proof of the representation result in Proposition 1, it is necessary to describe the behavior of the different components of $d V_{\tau} / V_{\tau-}$. First, let the dynamics of $d V_{\tau} / V_{\tau-}$ be rewritten using $\beta_{c, t}=w_{c, t}-\theta_{c, t}$ along with Itô's product rule as

$$
\begin{aligned}
\frac{d V_{\tau}}{V_{\tau-}} & =\sum_{c=0}^{C} w_{c, t} \frac{d P_{c, \tau}}{P_{c, \tau-}}+\beta_{c, t} \frac{d S_{c, \tau}}{S_{c, \tau-}}+\theta_{c, t}\left(\lambda_{0, \tau}-\lambda_{c, \tau}\right)+w_{c, t}\left[d P_{c, \tau} / P_{c, \tau}, d S_{c, \tau} / S_{c, \tau}\right]+o_{p}(d \tau) \\
& =\sum_{c=0}^{C} w_{c, t} \frac{d P_{c, \tau}}{P_{c, \tau-}}+\beta_{c, t} \frac{d S_{c, \tau}}{S_{c, \tau-}}+\tilde{\Sigma}_{c, \tau}^{h, 3} d \tau+w_{c, t} \tilde{d}_{c, \tau} \tilde{k}_{c, \tau} d L_{c, \tau}+o_{p}(d \tau)
\end{aligned}
$$

where $\tilde{\Sigma}_{c, \tau}^{h, 3}=\theta_{c, t}\left(\lambda_{0, \tau}-\lambda_{c, \tau}\right)+w_{c, t} \sigma_{c, \tau} \varphi_{c, \tau} \psi_{c, \tau}$ and the second equality follows by independence of the (potential) jumps $\left(d N_{\tau}, d M_{\tau}\right)$ and the Brownian increments $\left(d W_{\tau}, d Y_{\tau}\right)$. Next, write (A.9) on vector form

$$
\frac{d V_{\tau}}{V_{\tau-}}=\boldsymbol{w}_{t}^{\prime} \frac{d \boldsymbol{P}_{\tau}}{\boldsymbol{P}_{\tau-}}+\boldsymbol{\beta}_{t}^{\prime} \frac{d \boldsymbol{S}_{\tau}}{\boldsymbol{S}_{\tau-}}+\tilde{\Sigma}_{\tau}^{h, 3} d \tau+\left(\boldsymbol{w}_{t} \cdot \tilde{\boldsymbol{d}}_{\tau} \cdot \tilde{\boldsymbol{k}}_{\tau}\right)^{\prime} d \boldsymbol{L}_{\tau}
$$

where $\tilde{\Sigma}_{\tau}^{h, 3}=\iota^{\prime} \tilde{\boldsymbol{\Sigma}}_{\tau}^{h, 3}$ and $\tilde{\boldsymbol{\Sigma}}_{\tau}^{h, 3}=\left(\tilde{\Sigma}_{0, \tau}^{h, 3}, \ldots, \tilde{\Sigma}_{C, \tau}^{h, 3}\right)^{\prime}$. Under Assumptions 1 and 3, it follows by applying Itô's lemma for jump-diffusions, see, e.g., Cont \& Tankov (2004, Proposition 8.14) or Øksendal \& Sulem (2007, Chapter 1), to the first component of (A.10) that

$$
\begin{aligned}
d \boldsymbol{p}_{\tau} & =\left(\boldsymbol{\mu}_{\tau}-\boldsymbol{\sigma}_{\tau} \cdot \boldsymbol{\sigma}_{\tau} / 2\right) d \tau+\boldsymbol{\sigma}_{\tau} \cdot d \boldsymbol{W}_{\tau}+\boldsymbol{d}_{\tau} \cdot d \boldsymbol{N}_{\tau}+o_{p}(d \tau) \\
& =\frac{d \boldsymbol{P}_{\tau}}{\boldsymbol{P}_{\tau-}}-\left(\boldsymbol{\sigma}_{\tau} \cdot \boldsymbol{\sigma}_{\tau} / 2\right) d \tau-\boldsymbol{d}_{\tau}^{e} \cdot d \boldsymbol{N}_{\tau}+o_{p}(d \tau), \quad \boldsymbol{d}_{\tau}^{e}=\tilde{\boldsymbol{d}}_{\tau}-\boldsymbol{d}_{\tau},
\end{aligned}
$$

that is, with $\boldsymbol{d}_{\tau}^{e}$ collecting the jump approximation error under the logarithmic transformation. Similarly for the second component of (A.10),

$$
d \boldsymbol{s}_{\tau}=\frac{d \boldsymbol{S}_{\tau}}{\boldsymbol{S}_{\tau-}}-\left(\boldsymbol{\varphi}_{\tau} \cdot \boldsymbol{\varphi}_{\tau} / 2\right) d \tau-\boldsymbol{k}_{\tau}^{e} \cdot d \boldsymbol{M}_{\tau}+o_{p}(d \tau), \quad \boldsymbol{k}_{\tau}^{e}=\tilde{\boldsymbol{k}}_{\tau}-\boldsymbol{k}_{\tau}
$$

Inserting (A.11) and (A.12) into (A.10) gives

$$
\begin{aligned}
\frac{d V_{\tau}}{V_{\tau-}}= & \boldsymbol{w}_{t}^{\prime}\left(d \boldsymbol{p}_{\tau}+\left(\boldsymbol{\sigma}_{\tau} \cdot \boldsymbol{\sigma}_{\tau} / 2\right) d \tau+\boldsymbol{d}_{\tau}^{e} \cdot d \boldsymbol{N}_{\tau}\right)+\boldsymbol{\beta}_{t}^{\prime}\left(d \boldsymbol{s}_{\tau}+\left(\boldsymbol{\psi}_{\tau} \cdot \boldsymbol{\psi}_{\tau} / 2\right) d \tau+\boldsymbol{k}_{\tau}^{e} \cdot d \boldsymbol{M}_{\tau}\right) \\
& +\Sigma_{\tau}^{h, 3} d \tau+\left(\boldsymbol{w}_{t} \cdot \tilde{\boldsymbol{d}}_{\tau} \cdot \tilde{\boldsymbol{k}}_{\tau}\right)^{\prime} d \boldsymbol{L}_{\tau}+o_{p}(d \tau) \\
= & \boldsymbol{w}_{t}^{\prime} d \boldsymbol{p}_{\tau}+\boldsymbol{\beta}_{t}^{\prime} d \boldsymbol{s}_{\tau}+\Sigma_{\tau}^{h, 3} d \tau+\tilde{J}_{\tau}^{e}+o_{p}(d \tau)
\end{aligned}
$$

where the third component $\Sigma_{\tau}^{h, 3}=\tilde{\Sigma}_{\tau}^{h, 3}+\boldsymbol{w}_{t}^{\prime}\left(\boldsymbol{\sigma}_{\tau} \cdot \boldsymbol{\sigma}_{\tau} / 2\right)+\boldsymbol{\beta}_{t}^{\prime}\left(\boldsymbol{\psi}_{\tau} \cdot \boldsymbol{\psi}_{\tau} / 2\right)$ collects Itô drift-change terms from (A.10)-(A.12) along with the interest rate differentials, and, similarly, the fourth component is comprised of the Itô jump approximations errors as well as (weighted) co-jumps between the country- 
wise equity indices and exchange rates against the USD, that is,

$$
\tilde{J}_{\tau}^{e}=\boldsymbol{w}_{t}^{\prime}\left(\boldsymbol{d}_{\tau}^{e} \cdot d \boldsymbol{N}_{\tau}\right)+\boldsymbol{\beta}_{t}^{\prime}\left(\boldsymbol{k}_{\tau}^{e} \cdot d \boldsymbol{N}_{\tau}\right)+\left(\boldsymbol{w}_{t} \cdot \tilde{\boldsymbol{d}}_{\tau} \cdot \tilde{\boldsymbol{k}}_{\tau}\right)^{\prime} d \boldsymbol{L}_{\tau}
$$

Next, decompose $d \boldsymbol{p}_{\tau}$ and $d \boldsymbol{s}_{\tau}$ into diffusive $(D)$ and jump parts as $d \boldsymbol{p}_{\tau}=d \boldsymbol{p}_{\tau}^{D}+\boldsymbol{d}_{\tau} \cdot d \boldsymbol{N}_{\tau}$ and $d \boldsymbol{s}_{\tau}=d \boldsymbol{s}_{\tau}^{D}+\boldsymbol{k}_{\tau} \cdot d \boldsymbol{M}_{\tau}$, respectively. Then (A.13) may be rewritten as

$$
\frac{d V_{\tau}}{V_{\tau-}}=\boldsymbol{w}_{t}^{\prime} d \boldsymbol{p}_{\tau}^{D}+\boldsymbol{\beta}_{t}^{\prime} d \boldsymbol{s}_{\tau}^{D}+\Sigma_{\tau}^{h, 3} d \tau+J_{\tau}+o_{p}(d \tau)
$$

where $J_{\tau}=\boldsymbol{w}_{t}^{\prime}\left(\tilde{\boldsymbol{d}}_{\tau} \cdot d \boldsymbol{N}_{\tau}\right)+\boldsymbol{\beta}_{t}^{\prime}\left(\tilde{\boldsymbol{k}}_{\tau} \cdot d \boldsymbol{N}_{\tau}\right)+\left(\boldsymbol{w}_{t} \cdot \tilde{\boldsymbol{d}}_{\tau} \cdot \tilde{\boldsymbol{k}}_{\tau}\right)^{\prime} d \boldsymbol{L}_{\tau}$. Applying Itô's lemma to a logarithmic transformation of (A.14) gives

$$
\begin{aligned}
d v_{t}= & \boldsymbol{w}_{t}^{\prime} d \boldsymbol{p}_{\tau}^{D}+\boldsymbol{\beta}_{t}^{\prime} d \boldsymbol{s}_{\tau}^{D}+\Sigma_{\tau}^{h, 3} d \tau-\left[\boldsymbol{w}_{t}^{\prime} d \boldsymbol{p}_{\tau}^{D}+\boldsymbol{\beta}_{t}^{\prime} d \boldsymbol{s}_{\tau}^{D}\right] / 2+f\left(V_{\tau-}, J_{\tau}, \boldsymbol{w}_{t}, \boldsymbol{\beta}_{t}\right) \\
& -f\left(V_{\tau-}, 0, \boldsymbol{w}_{t}, \boldsymbol{\beta}_{t}\right)+o_{p}(d \tau) \\
= & \boldsymbol{w}_{t}^{\prime}\left(d \boldsymbol{p}_{\tau}^{D}+\boldsymbol{\lambda}_{0, \tau} d \tau-\boldsymbol{\lambda}_{\tau} d \tau\right)+\boldsymbol{\beta}_{t}^{\prime}\left(d \boldsymbol{s}_{\tau}^{D}-\boldsymbol{\lambda}_{0, \tau} d \tau+\boldsymbol{\lambda}_{\tau} d \tau\right)+\Sigma_{\tau}^{h, 1} d \tau+\Sigma_{\tau}^{h, 2} d \tau \\
& +f\left(V_{\tau-}, J_{\tau}, \boldsymbol{w}_{t}, \boldsymbol{\beta}_{t}\right)-f\left(V_{\tau-}, 0, \boldsymbol{w}_{t}, \boldsymbol{\beta}_{t}\right)+o_{p}(d \tau)
\end{aligned}
$$

where the first equality follows since the quadratic (co)-variation of $\Sigma_{\tau}^{h, 3}$ vanishes asymptotically by the latter being locally bounded, càdlàg, and $\mathcal{F}_{\tau}$-adapted under Assumptions 1-2. The second equality follows by rewriting the drift contribution of $\Sigma_{\tau}^{h, 3}$ using the definitions of $\Sigma_{\tau}^{h, 1}$ and $\Sigma_{\tau}^{h, 2}$ from the proof of Proposition 1, which collect components from the diffusive parts of $d \boldsymbol{p}_{\tau}$ and $d \boldsymbol{s}_{\tau}$ only.

As the last step, suppose $\left|J_{\tau}\right|<1$, then the discontinuous part of (A.15) may be written

$$
\begin{aligned}
f\left(V_{\tau-}, J_{\tau}, \boldsymbol{w}_{t}, \boldsymbol{\beta}_{t}\right)-f\left(V_{\tau-}, 0, \boldsymbol{w}_{t}, \boldsymbol{\beta}_{t}\right) & =\ln \left(V_{\tau-}\left(1+J_{\tau}\right)\right)-\ln \left(V_{\tau-}\right)=\ln \left(1+J_{\tau}\right) \\
& =J_{\tau}+\sum_{\ell=2}^{\infty}(-1)^{(\ell-1)} \frac{J_{\tau}^{\ell}}{\ell}
\end{aligned}
$$

using a standard Taylor expansion. Hence, applying (A.16) in conjunction with (A.15) and addition and subtraction of $\boldsymbol{w}_{t}^{\prime}\left(\boldsymbol{d}_{\tau} \cdot d \boldsymbol{N}_{\tau}\right)+\boldsymbol{\beta}_{t}^{\prime}\left(\boldsymbol{k}_{\tau} \cdot d \boldsymbol{N}_{\tau}\right)$ gives

$$
d v_{t}=\boldsymbol{w}_{t}^{\prime}\left(d \boldsymbol{p}_{\tau}+\boldsymbol{\lambda}_{0, \tau} d \tau-\boldsymbol{\lambda}_{\tau} d \tau\right)+\boldsymbol{\beta}_{t}^{\prime}\left(d \boldsymbol{s}_{\tau}-\boldsymbol{\lambda}_{0, \tau} d \tau+\boldsymbol{\lambda}_{\tau} d \tau\right)+\Sigma_{\tau}^{h} d \tau+J_{\tau}^{e}+o_{p}(d \tau)
$$

where $\Sigma_{\tau}^{h}=\Sigma_{\tau}^{1, h}+\Sigma_{\tau}^{2, h}$ and $J_{\tau}^{e}=\tilde{J}_{\tau}^{e}+\sum_{\ell=2}^{\infty}(-1)^{(\ell-1)} J_{\tau}^{\ell} / \ell$, providing the final representation. 
2015-46: $\quad$ Asger Lunde, Anne Floor Brix and Wei Wei: A Generalized Schwartz Model for Energy Spot Prices - Estimation using a Particle MCMC Method

2015-47: Annastiina Silvennoinen and Timo Teräsvirta: Testing constancy of unconditional variance in volatility models by misspecification and specification tests

2015-48: $\quad$ Harri Pönkä: The Role of Credit in Predicting US Recessions

2015-49: $\quad$ Palle Sørensen: Credit policies before and during the financial crisis

2015-50: $\quad$ Shin Kanaya: Uniform Convergence Rates of Kernel-Based Nonparametric Estimators for Continuous Time Diffusion Processes: A Damping Function Approach

2015-51: Tommaso Proietti: Exponential Smoothing, Long Memory and Volatility Prediction

2015-52: $\quad$ Mark Podolskij, Christian Schmidt and Mathias Vetter: On U- and V-statistics for discontinuous Itô semimartingale

2015-53: $\quad$ Mark Podolskij and Nopporn Thamrongrat: A weak limit theorem for numerical approximation of Brownian semi-stationary processes

2015-54: $\quad$ Peter Christoffersen, Mathieu Fournier, Kris Jacobs and Mehdi Karoui: Option-Based Estimation of the Price of Co-Skewness and Co-Kurtosis Risk

2015-55 Kadir G. Babaglou, Peter Christoffersen, Steven L. Heston and Kris Jacobs: Option Valuation with Volatility Components, Fat Tails, and Nonlinear Pricing Kernels

2015-56: $\quad$ Andreas Basse-O'Connor, Raphaël Lachièze-Rey and Mark Podolskij: Limit theorems for stationary increments Lévy driven moving averages

2015-57: $\quad$ Andreas Basse-O'Connor and Mark Podolskij: On critical cases in limit theory for stationary increments Lévy driven moving averages

2015-58: $\quad$ Yunus Emre Ergemen, Niels Haldrup and Carlos Vladimir Rodríguez-Caballero: Common long-range dependence in a panel of hourly Nord Pool electricity prices and loads

2015-59: $\quad$ Niels Haldrup and J. Eduardo Vera-Valdés: Long Memory, Fractional Integration, and Cross-Sectional Aggregation

2015-60: Mark Podolskij, Bezirgen Veliyev and Nakahiro Yoshida: Edgeworth expansion for the pre-averaging estimator

2016-01: $\quad$ Matei Demetrescum, Christoph Hanck and Robinson Kruse: Fixed-b Inference in the Presence of Time-Varying Volatility

2016-02: $\quad$ Yunus Emre Ergemen: System Estimation of Panel Data Models under LongRange Dependence

2016-03: Bent Jesper Christensen and Rasmus T. Varneskov: Dynamic Global Currency Hedging 